\title{
Electroanalytical methods and advanced characterization techniques for novel ion battery anode research
}

Received 00th January 20xx, Accepted 00th January 20xx DOI: $10.1039 / \times 0 \times x 00000 x$

\begin{abstract}
Jianming Zhao, ${ }^{\mathrm{a}, \mathrm{e}_{+}}$Manuel Cano, ${ }^{\mathrm{b}}+$ Juan J. Giner-Casares, ${ }^{\mathrm{b}}$ Rafael Luque*c ${ }^{*}$ and Guobao Xu*a
The physicochemical properties of the metal-ion battery anodes display a key role in the full behavior and electrochemical performance of energy storage devices. Novel ion battery anode materials (NIBAMs) are attracting a growing attention due to the capacity limitation of the classic graphite anodes, which provide low specific- and rate-capacity, and safety issues. Nevertheless, the electrochemical performance of NIBAMs such as the capacity, cyclability, rate capability, voltage profiles, and safety are strongly dependent on the structural and morphological evolution, phase transformation, ion diffusion, and electrode/electrolyte interface reconstruction during charge-discharge cycling and storage. Therefore, in-depth understanding of the structure and kinetics of NIBAMs and the electrode/electrolyte interfaces is essential for optimizing their preparation and application conditions, and exploring NIBAMs. Traditional electroanalytical methods, such as charge/discharge, cyclic voltammetry, and electrochemical impedance spectroscopy (EIS) are utilized to research the capacity, resistance, rate capability and cyclability of NIBAMs. Recently, rapid progress and development in hyphenated techniques by coupling with X-ray, electron microscopy, scanning probe microscopy, optical techniques, neutron techniques, and magnetic techniques have provided extensive insights into the nature of structural evolution, morphological changes of NIBAMs, and electrode/electrolyte interface. In this review, a comprehensive overview of both classical electroanalytical methods and advanced (in situ/operando) characterization techniques for studying the NIBAMs is provided.
\end{abstract}

\section{Introduction}

High-performance rechargeable batteries are urgently required by swiftly expanding technologies, mainly portable electronic devices (PEDs, e.g., mobile phones, smart watches, tablets, computers) as well as electric vehicles (EVs, e.g. motorbikes, cars, buses). ${ }^{1-4}$ The challenge of global warming and the advance of renewable energy sources demands adequate energy storage systems, which can exchange electrical into chemical energy, with high efficiency and reversibility. ${ }^{5,} 6$ Lithium-ion batteries (LIBs) have emerged as promising energy storage systems for the different applications above. ${ }^{1,7}$ The high energy density and storage efficiency together with the economic maintenance and flexibility are some of the more important strengths of this technology. ${ }^{8}$ Considering however the limited amount of lithium in the earth and the long-term health effects related to waste exposure, sodium-ion (NIBs) and potassium-ion (PIBs) batteries are budding as attractive alternatives due to their sustainability and abundance in nature. ${ }^{9-13}$

\footnotetext{
a. State Key Laboratory of Electroanalytical Chemistry, Changchun Institute of Applied Chemistry, Chinese Academy of Sciences, Changchun, Jilin 130022, People's Republic of China. E-mail: guobaoxu@ciac.ac.cn

b. Departamento de Química Física y Termodinámica Aplicada, Instituto Universitario de Nanoquímica (IUNAN), Universidad de Córdoba (UCO), Campus de Rabanales, Ed. Marie Curie (C-3), Ctra Nnal IV-A, Km 396, Córdoba, E-14014, Spain.

Departamento de Química Orgánica, Universidad de Cordoba, Campus de Rabanales, Ed. Marie Curie (C-3), Ctra Nnal IV-A, Km 396, Córdoba, E-14014, Spain.E-mail: rafael.luque@uco.es

d. Peoples Friendship University of Russia (RUDN University), 6 Miklukho Maklaya str., 117198, Moscow, Russia.

e. Development Center of Shenzhen, Division 2 of BYD, 16 Xiusha Rode, Pingshan District, Shenzhen, Guangdong 518118, China.

+ These authors contributed equally to this work.
}

A top challenge for energy storage devices is providing superior energy conversion and simultaneously being able to be packed into the minimum weight and volume. Therefore, volumetric and specific capacities are high-value parameters to consider beyond purely physicochemical performance. ${ }^{14}$ Intercalation and de-intercalation of metal ions into the active materials at the electrodes is the basic design for these devices, making the use of different anodes instead of graphite the main goal, focusing on reducing the electrode volume with a significant increase of the capacity. An excellent review of the novel ion battery anode materials (NIBAMs) was published by Qi et al., ${ }^{14}$ pointing out their advantages and limitations. Table 1 shows a tentative classification of the different types of NIBAMs. ${ }^{14-21}$

Highly pursued features of NIBAMs include high electrical conductivity, chemically inert, resistance to corrosion, mechanical resistance, high electrochemically active area, high specific surface area, biocompatible, durability, and low-cost, to name only the forefront research topics. The quantitative assessment of such critical parameters in all anode materials including of course NIBAMs has been performed using different electroanalytical methods. A comprehensive classification into traditional electroanalytical methods and advanced characterization techniques is presented herein, including illustrative examples. A thorough discussion on the actual strength and weakness of each method has been included, in the spirit of helping researchers to select those methods that match their requirements and capabilities. The current trends in the field are discussed, which in our opinion is rapidly shifting towards the combination of in situ/operando characterization techniques for studying the NIBAMs performance during charging/discharging or storage. 
Table 1. Tentative classification of different types of NIBAMs. ${ }^{14,15}$

\begin{tabular}{|c|c|c|}
\hline & Subtypes/Examples & $\begin{array}{c}\text { Advantages (A) and } \\
\text { disadvantaged } \\
\text { (D) }\end{array}$ \\
\hline \multirow[t]{4}{*}{ Carbon-based } & 0D fullerenes & \multirow{4}{*}{$\begin{array}{l}\text { A: Low-cost, } \\
\text { hierarchical structure } \\
\text { and high electronic } \\
\text { conductivity. } \\
\text { D: Safety issues, low } \\
\text { rate and specific } \\
\text { capacities }\end{array}$} \\
\hline & 1D CNTs & \\
\hline & 2D Graphene & \\
\hline & 3D Networks & \\
\hline \multirow[t]{2}{*}{ Lithium-based } & Li-C & \multirow{2}{*}{$\begin{array}{l}\text { A: High capacities. } \\
\text { D: Environmental } \\
\text { impact. }\end{array}$} \\
\hline & Li-metal & \\
\hline Silicon-based & $\begin{array}{l}\text { a-Si } \\
\mathrm{c}-\mathrm{Si} \\
\mathrm{Si} \text { nanowires }\end{array}$ & $\begin{array}{l}\text { A: Low-cost, clean } \\
\text { resources and high } \\
\text { specific capacity. } \\
\text { D: Large volume } \\
\text { change. }\end{array}$ \\
\hline Alloy-based & $\begin{array}{l}\mathrm{Sn}, \mathrm{Sb}, \mathrm{Al}, \mathrm{Mg}, \mathrm{Ag} \text {, and } \\
\text { their alloys }\end{array}$ & $\begin{array}{l}\text { A: Good security and } \\
\text { high specific } \\
\text { capacity. } \\
\text { D: Low electronic } \\
\text { conductivity and } \\
\text { large volume change. }\end{array}$ \\
\hline $\begin{array}{l}\text { Transition Metal } \\
\text { Oxides (TMOs) }\end{array}$ & $\begin{array}{l}\mathrm{MoO}_{\mathrm{x}}, \mathrm{WO}_{\mathrm{x}}, \mathrm{Co}_{\mathrm{x}} \mathrm{O}_{\mathrm{y}}, \\
\mathrm{Fe}_{\mathrm{x}} \mathrm{O}_{\mathrm{y}}, \mathrm{ZnO}_{\mathrm{x}}, \mathrm{TiO}_{2} \text {, and } \\
\text { combinations }\end{array}$ & $\begin{array}{l}\text { A: High specific } \\
\text { capacity and good } \\
\text { stability. } \\
\text { D: Large potential } \\
\text { hysteresis and low } \\
\text { coulombic } \\
\text { efficiency. }\end{array}$ \\
\hline $\begin{array}{l}\text { Transition Metal } \\
\text { Chalcogenides } \\
\text { (TMCs) }\end{array}$ & $\mathrm{CuS}, \mathrm{SnS}_{2}, \mathrm{TiS}_{2}$ & $\begin{array}{l}\text { A: High electrical } \\
\text { conductivity } \\
\text { theoretical and } \\
\text { capacity. } \\
\begin{array}{l}\text { D: Affinity with } \\
\text { soluble polysulfides. }\end{array}\end{array}$ \\
\hline $\begin{array}{c}\text { Composites/Hybrid } \\
\text { structures }\end{array}$ & $\begin{array}{l}\text { Graphene-based, } \\
\text { Carbon black based, } \\
\text { etc. }\end{array}$ & $\begin{array}{l}\text { A: Better electron } \\
\text { transfer and } \\
\text { electrochemical } \\
\text { performance. } \\
\text { D: Limited } \\
\text { pulverization of } \\
\text { active materials. }\end{array}$ \\
\hline
\end{tabular}

\section{Traditional electroanalytical methods}

Traditional electroanalytical methods usually include galvanostatic charge/discharge, cyclic voltammetry and electrochemical impedance spectroscopy.

\subsection{Galvanostatic charge/discharge}

Characterization of metal-ion batteries involves galvanostatic charge/discharge measurements during various cycles. During the charge process, the metal ions of the battery flow from the cathode to the anode through the electrolyte and the membrane, whilst in the discharge reaction the direction reverses and the ions flow from the negative to the positive electrode (Figure 1). Briefly, chronopotentiometric and chronoamperometric experiments are recorded at several ranges for a battery system (i.e. potential and current vs. time plots) and subsequently, potential versus capacity plots can be calculated. The latter analysis provides the $\mathrm{C}$-rate that is a measure of the rate at which a battery is charged or discharged relative to its maximum capacity. C-rate is used as a ranking on batteries to indicate the maximum current that a whole cell can safely deliver on a circuit. Together with the cyclic voltammetry, this is the most frequently used electroanalytical method for the determination of the specific capacitance of the battery. Additionally, by these galvanostatic charge/discharge curves, important quantitative information can be found on the metal-ion transport kinetic, and on the electrochemical performance and efficiency of the whole cell, as well as its dependence on usage and environmental factors (e.g. temperature, state-of-charge, etc.).

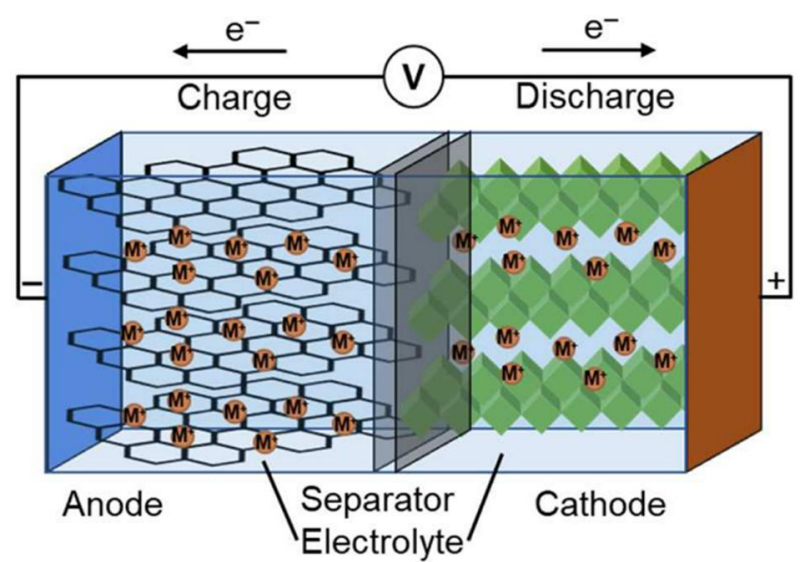

Figure 1. Schematic representation of a metal-ion battery. During the charge, the metal ions are deinserted from the cathode and deposited in the anode, whilst the electrons flow across the external circuit.

Due to the enormous amount of research works employing this characterization technique, only the first and last ones will be included in this section. As a representative example, in 1999, Robertson et al. reported one of the first studies investigating the potential use of $\mathrm{Li}_{1+\mathrm{x}} \mathrm{Fe}_{1-3 \mathrm{x}} \mathrm{Ti}_{1+2 \mathrm{x}} \mathrm{O}_{4}(0.0 \leq \mathrm{x} \leq 0.33$, LFTO $)$ spinels as TMO NIBAM for LIBs. ${ }^{22}$ Using galvanostatic cycling at a potential range of 0.01$2.6 \mathrm{~V}$ vs. $\mathrm{Li} / \mathrm{Li}^{+}$, they investigated the electrochemical behaviour and the associated structural changes of these NIBAMs. They concluded that samples containing Fe provided worse reversible capacity, which was attributed to the increase of the level of cation disorder in the spinel-type structure with the iron content. Almost twenty years later, Lin et al. also used classical galvanostatic charge-discharge curves to infer the electrochemical performance (i.e. specific capacity, rate performance and capacity retention) of a $3 \mathrm{D}$ graphene nanowallnanocrystalline Si nanocomposite (GNWs@Si) NIBAM for LIBs. ${ }^{23}$ They performed a comparative study of pure silicon and GNWs@Si composite, using a potential range of $0.001-1.5 \mathrm{~V}$ vs. $\mathrm{Li} / \mathrm{Li}^{+}$, at a rate of $0.1 \mathrm{C}$ during the first three cycles and at a rate of $1 \mathrm{C}$ during the following ones (Figure 2A-D). They showed that the GNWs structure was not destroyed during the cycling and concluded that the increased capacity retention of the GNWs@Si-based NIBAM could be attributed to the graphene preparation onto Ni foam matrix, which provides 3D network structure and excellent conductivity to the whole.

Galvanostatic charge-discharge measurements is also suitable for SIBs and PIBs. For instance, Xie et al. used this traditional electroanalytical technique to examine the cycling performance of $\mathrm{Na}_{2} \mathrm{Ti}_{3} \mathrm{O}_{7} @$ Carbon hollow spheres as NIBAM in SIBs. ${ }^{24}$ They used a 
voltage range of $0.01-2.5 \mathrm{~V}$ versus $\mathrm{Na} / \mathrm{Na}^{+}$for the measurements at the current density of $1 \mathrm{C}$. They showed that $\mathrm{Na}_{2} \mathrm{Ti}_{3} \mathrm{O}_{7} @ \mathrm{CHHSs}$ exhibited reversible capacities of 210, 179, 142, 120, 94, 82, and 63 $\mathrm{mAh} \mathrm{g}^{-1}$ at the current densities of $1,2,5,10,20,30$, and $50 \mathrm{C}$, respectively. In addition, this NIBAM showed a good durability and the best rate performance ever reported. They concluded that both the improved conductivity and $\mathrm{Na}$ ion diffusion property were conferred by the N-doped carbon-coated ultrathin nanosheet structure. Whilst for PIBs, Zheng et al. employed galvanostatic charge-discharge measurements to assess the C-rate performance and cycling stability of composite/hybrid structure based NIBAM, constituted by ultrathin sodium titanate (NTO)/potassium titanate (KTO) nanosheets sandwiched between graphene layers (i.e. a sandwich-type electrode). ${ }^{25}$ They demonstrated that $\mathrm{KTO} / \mathrm{rGO}-10 \%$ provided the following reversible capacities of $228,162,116$ and $84 \mathrm{~mA} \mathrm{~h} \mathrm{~g}^{-1}$ at the current densities of $0.1,0.2,0.5,1 \mathrm{~A} \mathrm{~g}^{-1}$, respectively (Figure 2E$\mathrm{H})$. Even at a high current density of $2 \mathrm{~A} \mathrm{~g}^{-1}$, this composite material exhibited a high reversible capacity of $75 \mathrm{~mA} \mathrm{~h} \mathrm{~g}^{-1}$ after 700 cycles, showing off the best reported electrochemical performance for PIBs.
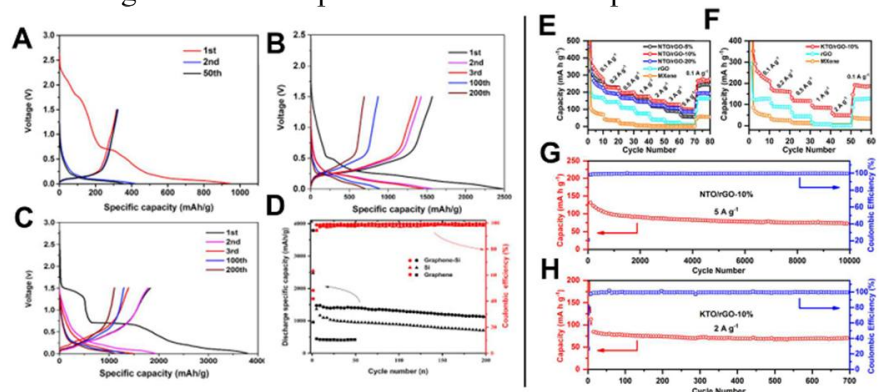

Figure 2. (A-D) Typical charge/discharge curves of the LIBs based on GNWs (A), pure silicon (B), and CNWs@Si composite (C); cycle performance of the LIBs based on the latter three samples (D). Reproduced with permission. ${ }^{23}$ Copyright 2019, Elsevier; (E-H) Electrochemical performance of the NTO/rGO films for SIBs and the KTO/rGO films for PIBs; (E) Comparison of rate performance of NTO with different rGO content, rGO and MXene at various current densities; (F) Comparison of rate performance of $\mathrm{KTO} / \mathrm{rGO}-$ $10 \%, \mathrm{rGO}$ and MXene at various current densities; $(\mathrm{G})$ Long-term cycling discharge capacities and Coulombic efficiencies of NTO/rGO-10 \% at $5 \mathrm{~A} \mathrm{~g}^{-1}$; (H) Long-term cycling discharge capacities and Coulombic efficiencies of $\mathrm{KTO} / \mathrm{rGO}-10 \%$ at $2 \mathrm{~A} \mathrm{~g}^{-1}$. Reproduced with permission. ${ }^{25}$ Copyright 2018 , Wiley.

As can be observed in the more recent reported studies, the tendency in the field is the development of NIBAMs type with composite/hybrid structures that combined materials with different composition, multidimensionality, porosity and redox features.

\subsection{Cyclic voltammetry (CV)}

$\mathrm{CV}$ is a type of potentiodynamic electrochemical measurement, in which the current density of a battery or cell is recorded during a potential range scanning at a given sweep rate and for a certain number of cycles. This traditional electroanalytical technique provides very useful information about not only the evolution of the electrochemical reactions or redox processes carried out, but also the battery's performance, including capacity and voltage limits as well as long-term behaviour. It is usually used in combination with the galvanostatic charge-discharge curves because it provides complementary information and is easily obtained by using the same potentiostat/galvanostat instrument.
In the first example mentioned above for LIBs and reported in 1999 by Robertson et al., ${ }^{22}$ they performed slow sweep CV curves for different compositions of LFTO NIBAMs at three potential ranges (Figure 3A). They observed a complicated electrochemical behaviour associated to the two redox couples (i.e. $\mathrm{Ti}^{4+} / \mathrm{Ti}^{3+}$ and $\mathrm{Fe}^{3+} / \mathrm{Fe}^{2+}$ ), and to the transition metals on both tetrahedral and octahedral sites. In the more recently reported example by Lin et al., ${ }^{23}$ they compared the $\mathrm{CV}$ curves obtained for GNWs@Si composite and for the individual constituents (i.e. pure silicon and GNWs) for the first three cycles and at a scanning rate of $0.1 \mathrm{mV} / \mathrm{s}$ (Figure 3B-D). The obtained results indicated that both GNWs and $\mathrm{Si}$ in the composite material supported the Li-insertion and Li-extraction. In addition, they observed that the oxidation peak gradually increased with cycling, suggesting that $\mathrm{Li}$ extraction kinetics was enhanced, and that the presence of Silicon withstood the volume change during lithiation-delithiation processes.
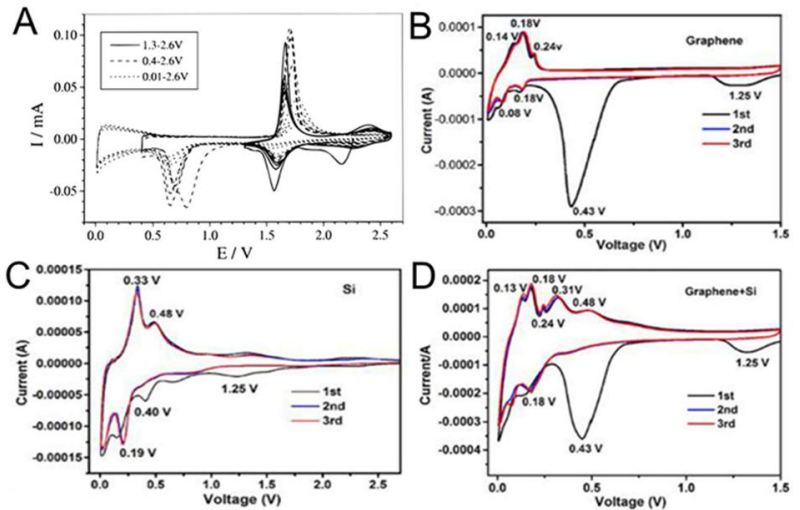

Figure 3. (A) $\mathrm{CV}$ curves of the $\mathrm{LIBs}$ based on $\mathrm{Li}_{1.2} \mathrm{Fe}_{0.4} \mathrm{Ti}_{1.4} \mathrm{O}_{4}(\mathrm{x}=0.2)$ at sweep rate of $20 \mu \mathrm{V} \cdot \mathrm{s}^{-1}$. Reproduced with permission. ${ }^{22}$ Copyright 1999, The Electrochemical Society, Inc.; (B-D) CVs of the LIBs based on GNWs (B), pure silicon (C) and GNWs@Si composite (D) for the first three cycles at scan rate of $0.1 \mathrm{mV} \cdot \mathrm{s}^{-1}$. Reproduced with permission. ${ }^{23}$ Copyright 2019, Elsevier.

Regarding the example above for SIBs, Xie et al. employed CV measurements to further investigate the origin of the superior electrochemical performance and the sodium ion storage mechanism of $\mathrm{Na}_{2} \mathrm{Ti}_{3} \mathrm{O}_{7} @ \mathrm{C}$ HHS NIBAM. ${ }^{24}$ For this, they measured the CV curves at different scan rates $\left(0.1-10 \mathrm{mV} \cdot \mathrm{s}^{-1}\right)$. At higher sweep rates than $2 \mathrm{mV} \cdot \mathrm{s}^{-1}$, a distortion from the basic shape of the $\mathrm{CV}$ curve was observed. They suggested that it may be originated by the increase of the Ohmic contribution and/or diffusion constraints. In addition, they calculated the contribution to the total charge stored resulting from the redox pseudocapacitance and intercalation. They demonstrated that the excellent performance was due to the high ratio of pseudocapacitive contribution in the total charge stored and the unique structure of hollow spheres assembled from N-doped carboncoated nanosheets. Whilst in the example above for PIBs, Zheng et al. employed $\mathrm{CV}$ curves (three first cycles, at potential range from 0 to $2.5 \mathrm{~V}$ vs. $\mathrm{K} / \mathrm{K}^{+}$and a scan rate of $0.1 \mathrm{mV} \mathrm{s}^{-1}$ ) to further confirm the high initial discharge capacity of $1094 \mathrm{mAh} \mathrm{g}^{-1}$ and low Coulombic efficiency about $24 \%$ of the proposed KTO/rGO- $10 \%$ NIBAM. ${ }^{25}$

Overall CV and galvanostatic charge-discharge are two traditional electroanalytical techniques that are always used together due to the complementarity of the obtained information and instrumentation required for the data acquisition. 


\subsection{Electrochemical impedance spectroscopy (EIS)}

EIS, also named AC impedance spectroscopy, is a characterization method for determining the small-signal impedance (or admittance) of the metal-ion batteries and subsequently for the modelling of their performance. EIS is used not only to characterize the kinetic mechanism of the charge transfer reaction that takes place at the electrode/electrolyte interface but also to determine the factors limiting the performance of an electrode, such as conductivity, charge-transfer properties, properties of the passivating layer, etc. For this measure, a small sinusoidal current (galvanostatic mode) or voltage (potentiostatic mode) are applied for a sweep of frequencies, whilst the amplitude and phase shift of the output voltage or current are recorded, respectively, obtaining the battery impedance spectrum. EIS measurements are usually performed in galvanostatic mode and for the frequency range of $10 \mathrm{kHz}-10 \mathrm{mHz}$. Additionally, EIS measurements are performed without superimposed DC current; and therefore, the influence of the current on the small-signal AC is not determined. ${ }^{26}$ EIS spectra (i.e. Nyquist plots; -Z" vs. $Z^{\prime}$ ) are often interpreted by using an electrical equivalent circuit (EEC), which is enough ambiguous and probably the major limitation for the practical application of this technique. An excellent book chapter collecting the different kinetic models for the lithium insertion/extraction into intercalation materials and the lithiation/delithiation of simple binary transition metal compounds was reported by Zhuang et al.. ${ }^{27}$
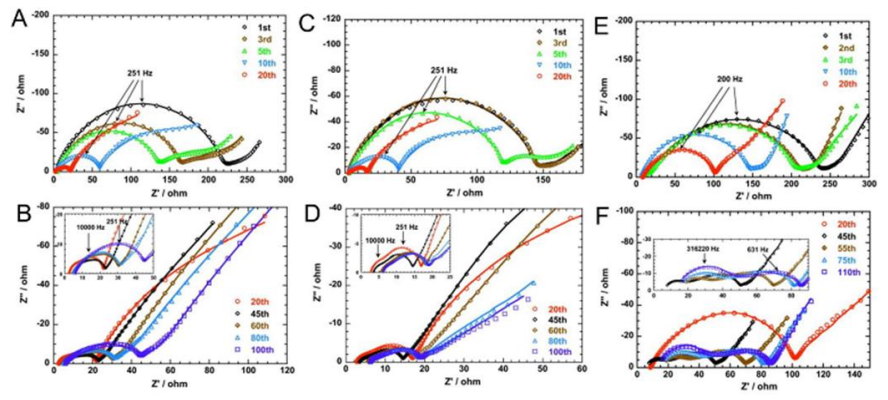

$\mathrm{G} \longrightarrow$

Figure 4. Nyquist plots of the annealed $(\mathrm{A}, \mathrm{B})$ and the carbonized $(\mathrm{C}, \mathrm{D}) \mathrm{Si}-\mathrm{C}$ electrodes with and without $(\mathrm{E}, \mathrm{F}) \mathrm{CNFs} / \mathrm{MWNT}$ after discharge in series of cycles. B, D and F-insets are the close-up of the high-frequency range. (G) The equivalent circuit used to model the impedance spectra, Reproduced with permission. ${ }^{28}$ Copyright 2011, Elsevier.

In 1999, $\mathrm{Li}$ et al. reported one of the first works using EIS to investigate the NIBAM passivation processes during cycling for LIBs. $^{29}$ In this study, nano-SnO and normal $\mathrm{SnO}$ were used as NIBAMs and subjected to EIS analysis at several discharged states, in order to infer the electrochemical mechanism sequence between replacement reaction and decomposition reaction (i.e. the SEI formation). They proposed three types of equivalent circuits that fitted the resulting EIS spectra at different discharge states, and discussed the spectra variations, charge-transfer resistance and double layer capacitance. They suggested that firstly a passivating film was formed on the surface of $\mathrm{SnO}$, and secondly the lithium ions that passed through the latter layer reacted with the NIBAM surface, producing both $\mathrm{Li}_{2} \mathrm{O}$ and fine grains of Li-Sn alloys. In 2006, Schranzhofer and co-workers reported a comparative EIS study about the SEI formation on graphite, and on Ni- and Pt-NIBAMs. ${ }^{30}$ They observed that the resistance of the SEI on graphite and nickel anodes showed similar time dependence, depicting a diffusion-controlled growth kinetic. They also performed temperature dependent measurements, yielding the activation energies for the ionic conduction of the SEI. In 2011, Guo et al. used EIS to investigate the capacity fading mechanism on three type of silicon-carbon composite NIBAMs. ${ }^{31}$ They showed that both the carbonization process during fabrication and the addition of different carbon nanofiber/nanotube additives affected seriously to the final cycling stability of Si-C NIBAM (Figure 4). They also highlighted that although the presence of additives could enhance the electronic conductivity of these anodes, it also induced a nonhomogeneous structure that decreased both the integrity and longterm cycling stability of the NIBAM. In 2013, Reddy et al. used EIS to understand variation resistance and capacitance value of different NIBAMs for LIBs, such as $\mathrm{CoN}, \mathrm{CoO}$, and $\mathrm{Co}_{3} \mathrm{O}_{4}$ in the form of nano$\mathrm{rod} /$ particles..$^{32} \mathrm{ESI}$ spectra were recorded for all the materials over the frequency range of $0.35 \mathrm{MHz}$ to $3 \mathrm{mHz}$, and with an AC amplitude of $10 \mathrm{mV}$. They observed that the overall impedance was in the following order: $\mathrm{Co}_{3} \mathrm{O}_{4}>\mathrm{CoO}>\mathrm{CoN}$. Although $\mathrm{CoO}$ and $\mathrm{CoN}$ showed the best performance, $\mathrm{CoN}$ provided a lower overall resistance than $\mathrm{CoO}$ and thus, the former material provided the best NIBAM kinetic. In 2014, Radvanyi et al. employed EIS to explain the rapid capacity fading of Silicon-based NIBAMs. ${ }^{33}$ Despite of using two distinct Si-based anodes and different experimental conditions, both systems showed a similar behaviour (i.e. SEI deposition around $50 \mathrm{kHz}$, charge transfer with a characteristic frequency from 300-1500 $\mathrm{Hz}$, and an inductive loop at around $1 \mathrm{~Hz}$ at potential below 0.35 vs $\mathrm{Li} / \mathrm{Li}^{+}$). They concluded that the inductive loop was associated to the constant SEI formation in competition with the charge transfer process. And more recently, in 2019, Mao et al. used EIS to further demonstrate the improved conductivity and electrons/ions transport of $\mathrm{Fe}_{3} \mathrm{O}_{4}$-embedded and $\mathrm{N}$-doped hierarchically porous carbon nanospheres $\left(\mathrm{Fe}_{3} \mathrm{O}_{4} @ \mathrm{~N}-\mathrm{HPCNs}\right)$ against its constituent components (i.e. N-HPCNs and $\mathrm{Fe}_{3} \mathrm{O}_{4}$ ). ${ }^{34}$ EIS curves for all the materials were measured at a frequency range from 0.01 to $100 \mathrm{kHz}$. They showed that $\mathrm{Fe}_{3} \mathrm{O}_{4} @ \mathrm{~N}-\mathrm{HPCN}$ and N-HPCNs NIBAMs presented reduced semicircle diameters, demonstrating their lower resistance and higher electrical conductivity. They also observed that the $\mathrm{Fe}_{3} \mathrm{O}_{4} @ \mathrm{~N}-\mathrm{HPCNs}$ anode was gradually activated, concluding that its superior electrochemical performance was associated to the large mesopores by buffering the huge volume change of $\mathrm{Fe}_{3} \mathrm{O}_{4} \mathrm{NPs}$ and avoiding their potential secondary aggregation.

As representative and recent examples in SIBs, Hu et al. used EIS to clarify the enhanced electrochemical performance of $\mathrm{Cu}_{3} \mathrm{Ge}$ coated by nitrogen-doped carbon $\left(\mathrm{Cu}_{3} \mathrm{Ge}-\mathrm{NC}\right)$ nanorods NIBAM. ${ }^{35}$ They employed the changes in charge transfer resistance $\left(R_{c t}\right)$ to further demonstrate the synergistic effect of the $\mathrm{Cu}_{3} \mathrm{Ge}-\mathrm{NC}$ composite components, which provided rapid electron transfer, fast reaction kinetics, and structural integrity to the whole. Guo and co-workers employed EIS to adjust the thickness of the capsular carbon shell encapsulating the $\mathrm{Sb}_{2} \mathrm{Se}_{3}$-based NIBAM for the best electrochemical performance. ${ }^{36}$ EIS measurements were recorded before and after 20th and 50th cycles at full-discharge state, using a perturbation voltage of $10 \mathrm{mV}$ in the frequency range of $100 \mathrm{KHz}$ to $0.01 \mathrm{~Hz}$. The sample with more b-cyclodextrin showed the smaller and more stable $R_{c t}$ value, indicating the easier charge transfer and more stable NIBAM. In addition, they calculated the Na ions diffusion coefficient of the different proposed NIBAMs by the Warburg coefficient in the 
low frequency range, demonstrating that the sample with most bcyclodextrin provided the highest value.

On the other hand, as examples for PIBs, Chang and co-workers reported the use of EIS to compare the electrochemical behaviour of a composite NIBAMs, containing red phosphorus dispersed on multiwalled carbon nanotubes $(\mathrm{RP} / \mathrm{C})$, and its individual constituent components. ${ }^{37}$ They demonstrated that the optimization factors to obtain high-performance anodes for PIBs were quite different from those for LIBs or NIBs, suggesting that the absence of phosphorouscarbon bonds could reduce the resistance of the alloying reaction between RP and potassium ions. Wang and co-workers used EIS to evaluate the electrode kinetics and to optimize the electrolyte composition of a RP-based NIBAM for PIBs. ${ }^{38}$ They proposed an electrolyte regulation strategy, which was applicable to other alloying anodes, to obtain for high-performance KIBs. For this, they employed potassium bis(fluorosulfonyl)imide salt-based carbonate (KFSI/EC) electrolyte as a salt-solvent complex that showed a moderate solvation energy, which alleviated side reactions between $\mathrm{K}$ metal and electrolyte, facilitating $\mathrm{K}$ ion diffusion-desolvation. EIS revealed that $1 \mathrm{M} \mathrm{KFSI/EC} \mathrm{in} \mathrm{diethyl} \mathrm{carbonate} \mathrm{electrolyte} \mathrm{exhibited} \mathrm{constant}$ impedance after 10 cycles and good cycling stability of the K metal, suggesting the formation of a stable and highly potassium ion conductive SEI layer on the RP/C alloying NIBAM.

\section{Advanced characterization techniques}

Although traditional electroanalytical methods provide objective data about the level of performance of the NIBAM for metal-ion batteries, these techniques showed an evident lack of information about internal electrochemical processes during cycling at atomic and/or nanometric scale. Instead, in operando or in situ characterization techniques allow a more detailed analysis by the direct monitoring of the different electrochemical process carried out into the NIBAMs during charge/discharge processes in cell batteries, such as changes in chemical states of constituent components, phase transformations, volume variations, formation of the solid electrolyte interphase (SEI) layers, dendrite generations, side reactions, constituents degradation, etc. One of the more important advantages of these in situ techniques is not only the information at real time of the processes occurring but also the absence of contamination factors induced by ex situ preparations. However, an evident improvement of the technical and technological facilities is still needed for this continuous streaming of data mainly due to the complex nature of the experimental set up, both by the conditions required for the battery cells and by the in situ characterization techniques. For instance, the electrochemical cell used for each in situ characterization method needs to provide not only appropriate windows and environmental conditions but also reproduce exactly the real battery working settings, in order to ensure that the information obtained is valid.

\subsection{X-ray}

X-ray analytical methods are used to research electronic and crystal structure information through scattering, spectroscopy and imaging techniques for investigating the electrochemical mechanism of NIBAMs.

\subsubsection{X-ray diffraction (XRD)}

Operando XRD is considered one of the best tools for investigating at real time structural evolution changes, phase transformations and volume changes of NIBAMs during the electrochemical processes. It is based on the scattering of X-rays by periodically spaced atoms in a crystal, producing a diffraction pattern that yields information about the crystallographic structure. In situ XRD experiments can be performed both on conventional diffractometers and at synchrotron radiation sources. The latter implies not only higher photon flux Xrays but also more complex data analysis and expensive costs than conventional XRD. ${ }^{39}$

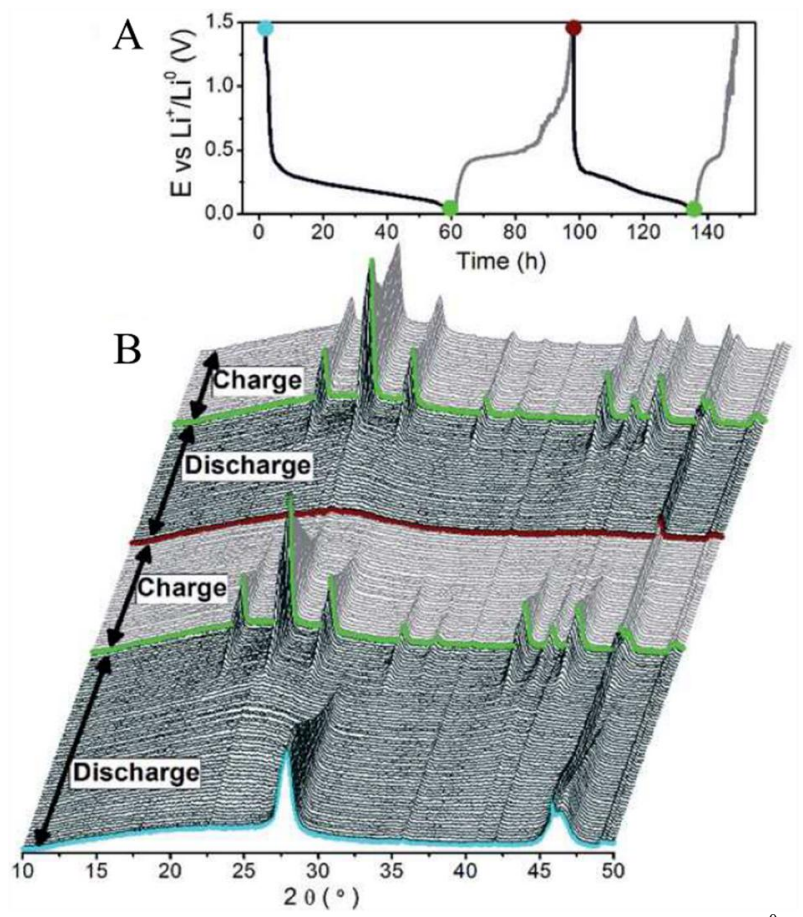

Figure 5. In situ XRD of a $\mathrm{Si}_{0.5} \mathrm{Ge}_{0.5}$ self-supported film cycled vs. $\mathrm{Li}^{0} / \mathrm{Li}^{+}$at the rate of $\mathrm{C} / 14$. Reproduced with permission. ${ }^{40}$ Copyright 2017, Royal Society of Chemistry.

Theoretically, the major limitation of this advanced characterization technique is the difficulty to obtain information about amorphous materials. However, in the bibliography several examples can be found using in situ XRD for monitoring the variations of noncrystalline anodes, such as silicon-based NIBAMs, during charge/discharge cycles (Figure 5). For instance, in 2004, Hatchard and Dahn performed in situ XRD to investigate the reaction mechanism of lithium with amorphous silicon (a-Si). ${ }^{41}$ They confirmed the formation of a new crystalline phase $\left(\mathrm{Li}_{15} \mathrm{Si}_{4}\right)$ only on a-Si films with thickness around $2 \mu \mathrm{m}$, and at potential below of 30 $\mathrm{mV}$ vs. $\mathrm{Li} / \mathrm{Li}^{+}$. Other illustrative example using silicon-based NIBAM is the research work reported by $\mathrm{Li}$ and co-workers in 2007, studying the reaction of lithium with crystalline $\mathrm{Si}$ (c-Si) composite electrode at room temperature. ${ }^{42}$ The provided information allowed them to build a phase diagram showing the phase changes and regions of phase stability during lithiation/delithiation reactions of this c-Si NIBAM. Later, Misra et al. using an X-ray transparent battery cell, performed in situ synchrotron XRD on silicon nanowires (SiNWs) during electrochemical cycling. ${ }^{43}$ The provided insight on the (de)lithiation mechanism and the phase evolution helped them to 
improve the cycling performance of SiNW NIBAMs by modifying its growing temperature.

This advanced electroanalytical method has been successfully used for other NIBAMs, such as composites/hybrid anode structures, alloybased anode materials, etc. For instance, quantitative measurements of elastic strains in a nanostructured silicon-coated nickel inverse opal scaffold anode were performed at different electrochemical cycling rates by in situ XRD monitoring the $\mathrm{Ni}$ peak-position and broadness. ${ }^{44}$ As it was expected, the observed increase in Ni lattice strain broadening during the silicon lithiation indicated that strains and stresses in the nanostructure were non-uniform due to the node and strut geometry of the inverse opal scaffold. In another study, using in situ X-ray diffraction, a high energetic source and an ionic liquid electrolyte was employed to monitor a gold thin-film model anode during the initial Li alloying and dealloying. ${ }^{45}$ The resulting data demonstrated that in these cyclic phase transitions six different crystalline alloy phases were involved. And more recently, this in situ method was used to investigate strain evolution in individual Gemicroparticles during electrochemical reaction with lithium. They observed a shift in position and a decrease in intensity of the crystalline Ge diffraction peak during the process, confirming that the expansion and plastic deformation during Li-reaction agreed with the mechanical model. ${ }^{46}$ Dong et al. also used in situ XRD to investigate the electrochemical reaction mechanism of 3D layered nanoarchitecture network of $\mathrm{Ni}\left(\mathrm{HCO}_{3}\right)_{2} / \mathrm{rGO}$ composites with highly uniform $\mathrm{Ni}\left(\mathrm{HCO}_{3}\right)_{2}$ nanocubes wrapped in $\mathrm{rGO}$ films. ${ }^{47}$ They observed that the 3D architecture provided a good conductivity network and was the responsible of the improvement storage performance of the Li-ions.

Furthermore, in situ XRD has not only been applied for LIBs but also for other novel metal-ion batteries, such as SIBs and PIBs, which are considered promising alternative candidates to the LIBs, especially for applications in large-scale energy storage systems. ${ }^{8}$ One of the first studies reported with SIBs was made in 2013 by $\mathrm{Yu}$ and co-workers, who studied the structure evolution of nanosized $\mathrm{Li}_{4} \mathrm{Ti}_{5} \mathrm{O}_{12}(\sim 44 \mathrm{~nm})$ upon sodium insertion. ${ }^{48}$ They demonstrated that chemical sodiation process was quite different to the well-known two-phase reaction of $\mathrm{Li}_{4} \mathrm{Ti}_{5} \mathrm{O}_{12} / \mathrm{Li}_{7} \mathrm{Ti}_{5} \mathrm{O}_{12}$ system during lithium insertion. In 2016, Hartung et al. employed operando synchrotron XRD to analyse the interplay between $\mathrm{Na}$ electrochemical and structural processes in alkali vanadates, such as $\mathrm{Na}_{2.46} \mathrm{~V}_{6} \mathrm{O}_{16}(\mathrm{NVO})$ and $\mathrm{Li}_{2.55} \mathrm{~V}_{6} \mathrm{O}_{16}$ (LVO). ${ }^{49}$ They concluded that the capacity decay increased with the ionic radius of the alkali ions $(\mathrm{K}+>\mathrm{Na}+>\mathrm{Li}+)$, indicating that smaller ions stabilized the structure. More recently, the operando XRD was used to infer the $\mathrm{Na}$ storage mechanisms of three different Bi-based NIBAMs (sputtered film, nanoporous and commercial) in SIBs. ${ }^{50}$ Surprisingly, despite of the different morphologies and sizes, all the Bi anodes followed the same two-step reversible alloying/dealloying mechanisms ( $\mathrm{Bi} \leftrightarrow \mathrm{NaBi} \leftrightarrow \mathrm{Na}_{3} \mathrm{Bi}$ ) during the discharge/charge processes, associated with two voltage plateaus. Similarly, for metalbased NIBAMs, Gao et al. proposed a novel channel-enhanced strategy, which was based on a nanoporous $\mathrm{Bi}_{50} \mathrm{Ni}_{50}$ alloy that contained intra-lattice straight ion channels and exhibited a superior electrochemical performance (i.e. specific capacity, rate capability, and cycling stability) as compared to nanoporous $\mathrm{Bi}_{75} \mathrm{Ni}_{2}$. Despite of the latter mentioned differences, both Bi-based NIBAMs share a similar $\mathrm{Na}$ storage mechanism. ${ }^{51}$ On the other hand, operando synchrotron-based X-ray powder diffraction (XRPD) was used by Xie and co-workers to verify that multi-shell antimony sulphide $\left(\mathrm{Sb}_{2} \mathrm{~S}_{3}\right)$ NIBAM presented much higher electrochemical activity (i.e. both volumetric energy density and durability) than pristine $\mathrm{Sb}_{2} \mathrm{~S}_{3}$ for SIBs. ${ }^{52}$ They suggested that the reported multi-shell $\mathrm{Sb}_{2} \mathrm{~S}_{3}$ approach may be generalized for the development of high-performance hollow structured NIBAMs to preserve high energy density.

Whilst as recent examples for PIBs, in 2018, Liu et al. used operando XRD to demonstrate that the few-layered antimony sulphide $\left(\mathrm{Sb}_{2} \mathrm{~S}_{3}\right)$ /carbon sheet composite NIBAM provided higher performance than the commercial bulk $\mathrm{Sb}_{2} \mathrm{~S}_{3}{ }^{53}$ The authors concluded that the $\mathrm{Sb}_{2} \mathrm{~S}_{3}$ minimized the volume expansion and enhanced the rate capability, whilst the carbon sheets provided electrical conductivity and maintained the stability of the whole composite. And, in 2019, Xie et al. combined operando synchrotronbased XRPD and ex situ X-ray absorption near-edge structure spectroscopy to understand the origin of the improved reversible capacity of a double-shell structured bismuth boxes for PIBs. ${ }^{54}$ They demonstrated that the larger surface area of this nanostructured alloybased NIBAM offered more sites to electrochemically alloy with potassium, resulting in a lower average oxidation state of bismuth.

\subsubsection{Coherent X-ray diffraction imaging (CXDI)}

Phase contrast and Bragg diffraction are combined in CXDI, which is a lens-less technique for $2 \mathrm{D}$ or $3 \mathrm{D}$ imaging reconstruction of nanostructures. ${ }^{55}$ In this technique a highly coherent beam of X-rays strikes on a sample and the beam scattered produces a diffraction pattern that is used to reconstruct an image via an iterative feedback algorithm. CXDI is a powerful, elegant, and rapidly developing technique that reconstructs $3 \mathrm{D}$ internal structure of microscopic objects with nanometric resolution from the fine structure of diffraction peaks, i.e. only by the wavelength of the X-rays and largest scattering angles measured. ${ }^{56} \mathrm{~A}$ great schematic representation of this $\mathrm{X}$-ray modality was reported by Cherukara et al. for measuring the strain state of an individual grain of copper $(\mathrm{Cu})$ in a polycristalline film during tensile loading (Figure 6). ${ }^{57}$ To analysis further, the resulting data were imported into an atomistic model, obtaining detailed information about the complex amplitude and strain variation in the crystal.

For LIBs, this technique in operando mode was previously applied to battery cathode materials based on a single $\mathrm{LiNi}_{0.5} \mathrm{Mn}_{1.5} \mathrm{O}_{4} \mathrm{NP}$ for monitoring both stripe morphologies and coherency strain at the nanoscale. ${ }^{58}$ They observed that $50 \mathrm{~nm}$ was the critical size for stripe formation. More recently, Quintero Cortes and co-workers combined synchrotron in situ XRD with CXDI to investigate the electrochemical reaction of individual Ge microparticles with $\mathrm{Li}^{46}$ They performed the direct measurement of the crystallographic strain evolution in individual particles, showing that the diffraction peak was shifted in position and diminished in intensity during reaction, suggesting the disappearance of the crystalline Ge phase.

Unfortunately, despite the enormous efforts made to date, in order to be accessible to all the scientific research community, this technique still needs a great technical, data analysis and computation 
developments for a high-quality image reconstruction. And, especially in in-situ environments, uncertainty due to thermal drift and vibrations can affect not only the position of particle/beam but also the incident angle, providing detrimental effects on the final reconstructed image.

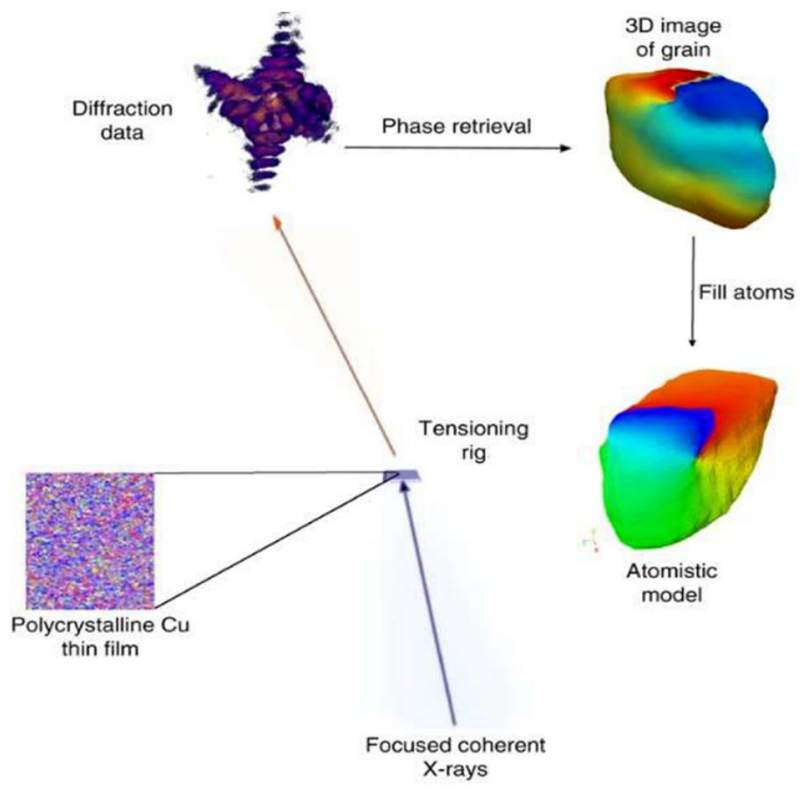

Figure 6. Coherent X-ray diffraction imaging in Bragg geometry. Focused coherent X-ray pulses were incident on the polycrystalline $\mathrm{Cu}$ sample. Diffracted X-ray pulses were recorded in the far-field by an ASI Timepix detector. The 3D speckle pattern at the (111) Bragg peak was recorded from 2$\mathrm{D}$ diffraction slices obtained by rocking the sample stage through small angles $(\sim 10)$. Reproduced with permission. ${ }^{57}$ Copyright 2018, Springer Nature.

\subsubsection{X-ray absorption spectroscopy (XAS)}

XAS is a synchrotron-based method that provides a direct measure of the chemical state of individual elements in a sample and can be used to track the oxidation state of the electrochemically active species of a wide range of components in a battery, such as NIMAMs, liquid electrolytes in addition to crystalline cathode electrodes. In a XAS experiment, the X-ray absorption coefficient of an element is determined when the photon energy is scanned below and above the absorption edge of an element associated with electrochemical reactions in batteries. ${ }^{59}$ XAS can be performed using hard and soft Xrays, $\mathrm{K}$ and $\mathrm{L}$ absorbance edges, full-field and scanning microscopes, and in 2D and tomographic imaging modes. ${ }^{55}$ In the XAS spectra two parts can be observed: X-ray near-edge spectroscopy (XANES) that covers the energy range up to $50-100 \mathrm{eV}$ near the absorption edge and gives the oxidation states and local symmetry changes of the absorbing element, and extended X-ray absorption fine structure (EXAFS) that covers several hundred $\mathrm{eVs}$, starting about $30 \mathrm{eV}$ above the absorption edge, and provides quantitative local structure information..$^{59}$

As representative examples for LIBs, in 2015, Lim et al. combined in situ XAS with XRD to study the Li-reaction pathways, phase transformations and local structural changes of crystalline Ge anodes on two different carbon-based conductive additives. ${ }^{60}$ They showed that Ge NIBAM on carbon nanotubes (CNTs) exhibited higher structural and electrochemical reversibility, as well as better stability in cycling, than those on carbon black. In 2016, Permien and coworkers used the same combination of X-ray methods to study the conversion reaction of $\mathrm{CoMnFeO}_{4} \mathrm{NPs}_{\text {in }} \mathrm{LIBs}^{61}{ }^{61}$ The combination of in situ XRD and XAS with other ex-situ techniques (such as TEM, ${ }^{7} \mathrm{Li}$ NMR, and molecular dynamics simulation) allowed them the identification of significant electronic and structural changes on different length scales. The finding indicated that the spinel NPs suffered a successive phase transition into a mixed monoxide due to the movement of the reduced cations from tetrahedral to octahedral positions. The same research group investigated the Li-reaction mechanisms of $\mathrm{CoFe}_{2} \mathrm{O}_{4}$ spinel NPs on reduced graphite oxide (rGO). ${ }^{62}$ They concluded that rGO acted as support for the NPs, preventing the electrical contact loss. In 2017, Loaiza et al. also used a combination of in situ techniques, such as XRD, XAS, Raman spectroscopy and ${ }^{7} \mathrm{Li} \mathrm{NMR}$, to explore the electrochemical mechanism of a $\mathrm{Si}_{0.5} \mathrm{Ge} 0.5$ alloy during lithiation/delithiation cycles. ${ }^{40}$ These complementary measurements gave them a whole view of the structural modifications induced by the charge-discharge processes. They concluded that $\mathrm{Si}$ and $\mathrm{Ge}$ were collectively active along the cycling process, with the formation of a ternary $\mathrm{Li}_{15}\left(\mathrm{Si}_{0.5} \mathrm{Ge}_{0.5}\right)_{4}$ phase (with a step of over lithiation) and with the recovery of the a-Si-Ge phase upon discharge and charge processes, respectively. Whilst Muller-Bouvet et al. used operando Manganese K-edge XAS spectra to study the redox mechanism of the $\mathrm{Li}_{7} \mathrm{MnN}_{4}$ NIBAM during $\mathrm{Li}$ extraction-insertion cycles. ${ }^{63}$ They used linear chemometric method, such as Principal Component Analysis (PCA) and Multivariate Curve Resolution-Alternating Least Squares (MCR-ALS), to quantitatively infer the profile evolutions of redox Mn species concentrations. The excellent reversibility of $\mathrm{Li}_{7} \mathrm{MnO}_{4}$ was also demonstrated at the local scale. The latter study pointed out the important need for data processing due to the large datasets provided for in situ spectroscopy methods (i.e. not only for XAS but also for Mössbauer spectroscopy and transmission soft X-ray microscopy). In this line, Fehse et al. have recently reported a review focus on the use of chemometric methods for the comprehensive analysis of these complex operando datasets from spectroscopy methods in battery materials. ${ }^{64}$

On the other hand, it is also necessary to highlight the research study reported by Fehse and co-workers, who measured operando TripleEdge XAS (i.e. at three different absorption edges simultaneously: Ti, $\mathrm{Sn}$, and $\mathrm{Sb}$ K-edge) to understand the electrochemical lithiation/delithiation mechanism of the ternary intermetallic $\mathrm{TiSnSb}$ NIBAM. ${ }^{65}$ The evolution of the XANES fingerprint and of the EXAFS spectra confirmed the reversibility of the conversion mechanism, revealing that Ti formed metallic NPs and binds back to both $\mathrm{Sn}$ and $\mathrm{Sb}$ upon lithiation and the following delithiation, respectively. Similarly, although in two different studies, Schmidt et al. required the information provided by $\mathrm{Fe}$ and $\mathrm{P}$ K-edges XAS to fully understand the high specific charge values obtained with diphosphonate-based organic-inorganic hybrid materials as NIBAM (i.e. lithium iron methylenediphosphonate, $\left.\mathrm{Li}_{1.4} \mathrm{Fe}_{6.8}\left[\mathrm{CH}_{2}\left(\mathrm{PO}_{3}\right)_{2}\right]_{3}\left[\mathrm{CH}_{2}\left(\mathrm{PO}_{3}\right)\left(\mathrm{PO}_{3} \mathrm{H}\right)\right] \cdot 4 \mathrm{H}_{2} \mathrm{O}, \quad \mathrm{LiFeMeDP}\right)$ that exceeded the theoretical maximum values. ${ }^{66,67}$ They demonstrated an additional contribution of reversible ligand co-cycling based on the $\mathrm{P}$ atoms of the diphosphonate ligands, upon delithiation occurring during the first potential plateau. ${ }^{67}$ 
Another remarkable and recent study was reported by $\mathrm{Yu}$ and coworkers, who used operando X-ray absorption imaging (XRI) to reveal the complex mechanistic steps of Li plating on Li-metal anodes that are considered an ideal NIBAM for Li rechargeable batteries. ${ }^{68}$ They showed the evolution of Li plating/stripping under operando and practical conditions, providing detailed insights into morphology evolution during Li plating. They also reported the effects of critical battery operating parameters on $\mathrm{Li}$ plating/stripping, such as concentration of Li salts, current density, ionic strength, and various electrolytes and additives.
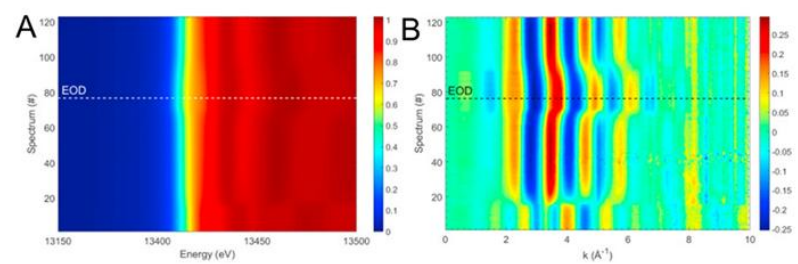

Figure 7. (A) XANES and (B) EXAFS operando BiL3-edge XAS spectra measured during the first electrochemical cycles of BiSb electrode vs. Na. The horizontal line labelled EOD represents the end of the first discharge. Reproduced with permission. ${ }^{69}$ Copyright 2019, Elsevier.

As representative examples for SIBs, in 2018, Fehse and co-workers combined simultaneously operando $\mathrm{Sn}$ and $\mathrm{Sb}$ K-edge XAS with ${ }^{119} \mathrm{Sn}$ Mössbauer spectroscopy to elucidate the better performance (i.e. enhanced cycle life and capacity retention) of SnSb in SIB than in LIB, in spite of its even larger volume expansion. ${ }^{70}$ After exhaustive data analysis using chemometric tools (PCA and MCR-ALS), the finding indicated that the sodiation reaction of $\mathrm{SnSb}$ was performed by a two-step process, clearly distinct from the Li-reaction, which was attributed to the gradual formation of amorphous, nano-confined intermediate phases and correlated elastic softening of highly sodiated $\mathrm{Sn}$ and $\mathrm{Sb}$ phases. More recently, the same research group combined operando $\mathrm{Sb}$ K-edge XAS and ${ }^{57} \mathrm{Fe}$ Synchrotron Mössbauer spectroscopy to clarify the role of $\mathrm{Sb}$ and $\mathrm{Fe}$ in the electrochemical sodiation mechanism of $\mathrm{FeSb}_{2}$ NIBAM. ${ }^{71}$ After analysing both datasets using an innovative chemometric approach, they concluded that Fe NPs played a key role in the reduction and stabilization of the polarization, as well as in the reversibility of the electrochemical sodiation of Sb. Using other Sb-based alloy, Darwiche at al. employed operando $\mathrm{Sb}$ K-edge and Bi L3-edge XAS to study the cycling sodiation mechanism of $\mathrm{Bi}_{0.50} \mathrm{Sb}_{0.50}$ (Figure 7). ${ }^{69,72}$ They showed that synchrotron beamline feasibilities allowed continuous and alternate measurements of XAS spectra of two elements with different energies of absorption edges (i.e. Sb K-edge at $30491 \mathrm{eV}$ and Bi L3-edge at $13418 \mathrm{eV}$ ). Chemometric methods were also required for the analysis of the collected data. Dixon et al. combined in operando and ex situ XAS to understand the difference in electrochemical mechanism in $\mathrm{SIB}$ and in LIB of bare $\mathrm{SnO}_{2}$ NIBAM. ${ }^{73}$ The poor electrochemical performance of this NIBAM in SIB was attributed to the incomplete conversion and the alloying reactions in the $\mathrm{Na} / \mathrm{SnO}_{2}$ half-cell.

\subsubsection{Transmission X-ray microscopy (TXM)}

TXM is a non-destructive 3D imaging approach to obtain a complete visual understanding of the electrochemical mechanisms on the NIBAMs during the electrochemical processes, such as structural evolution, morphological changes, volume expansion, dendrite formation, etc. In TXM a relatively large X-ray beam is focused on to the sample and the transmitted X-rays are collected and concentrated to a detector by an objective lens (Figure 8). ${ }^{39}$ One of the major advantages of TXM is that it does not require vacuum conditions, and thus, aqueous electrolytes are feasible..$^{74}$ In addition, it can image thicker samples than TEM because X-rays penetrate through most samples, and TXM overcome in situ TEM's limitations of high spatial resolution within a small field of vision. ${ }^{75}$

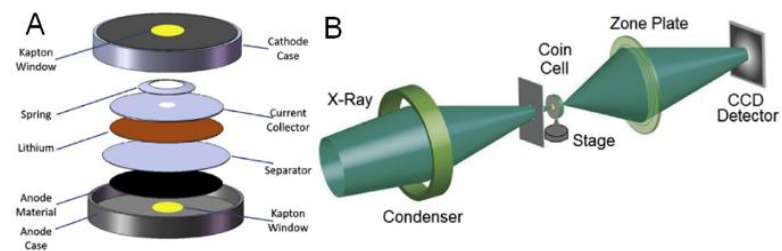

Figure 8. Representative schematic representations of the in operando coin cell assembly (A) and the synchroton transmission X-ray microscopy setup (B). Reproduced with permission. ${ }^{76}$ Copyright 2019, Elsevier.

For LIBs, the first study was reported in 2010 by Chao et al., who used in situ TXM to study the evolution of the interior structure of a Sn particle, as working alloy anode, which provides a high X-ray contrast. ${ }^{77}$ After the first lithiation cycle, they observed the formation of porous Sn particles due to metal recrystallization. They concluded that the use of alloying particles with smaller size and ductile nature was beneficial for dimensionally stable LIBs (i.e. it reduced the expansion volume, increased the kinetic and minimized the particle pulverization problem). One year later, this same research group used in situ TXM to investigate the evolution of the interior microstructures of SnO during lithiation/de-lithiation processes. ${ }^{78}$ They observed the formation of a porous secondary $\mathrm{SnO}$ particle, which suffered reversible deformations during subsequent cycles, providing better electrochemical performances than with dense Sn particle. These authors also reported the use of in situ TXM and XRD in combination by synchrotron sources to study the evolution of interior microstructures of $\mathrm{Sn}$ and $\mathrm{SnSb}$ particles during the first lithiation/delithiation cycles. ${ }^{79}$ They showed that both particles exhibited different classes of electrochemical behaviours, concluding that microstructures and deformation rates were dependent on particle composition, size, and alloy stoichiometry with Li. Also, in 2011, Meirer and co-workers proposed a new X-ray imaging set-up combining full-field TXM with XANES (named TXM-XANES) to follow $2 \mathrm{D}$ and $3 \mathrm{D}$ morphological and chemical changes in large volumes at nanometric resolution. ${ }^{80}$ Using a $\mathrm{NiO}$ thin-film anode as a proof-of-concept, they demonstrated that TXN-XANES provided direct information about the chemical composition, morphology and porosity of this NIBAM, converting this combination as an ideal tool for investigating nanoscale reactions and phase transformations in complex systems.

In 2014, Weker et al. combined in operando TXM and nanotomography to understand the origin, dynamics, and failure mechanisms of Ge NIBAM during cycling. ${ }^{81}$ They observed a clear size-dependent behaviour of Ge particles during lithiation, the large particles ( $>$ microns) displayed cracks whilst the small ones suffered a rapid loss of electrical contact. In 2017, Cook and co-workers employed operando TXM to investigate the mechanisms that control the enhanced cycling stability of nanoporous $\mathrm{Sn}^{82}$ They noted that 
this nanoporous material showed a 6-fold smaller areal expansion after lithiation than non-porous one, which was associated to the internal porosity and unique nanostructure. In the same year, Cheng et al. used operando TXM to visualize the lithium dendrite growth dynamics on $\mathrm{Cu}$ surface. ${ }^{83}$ They observed that the aspect ratio (height/width) of the Li-coating was dependent on the applied charge and that a non-homogeneous current distribution induced a serious dendritic formation. More recently, $\mathrm{Li}$ and co-workers employed operando synchrotron transmission TXM to visualize the morphological evolution of Sn particles with different sizes during the first lithiation/delithiation cycles. ${ }^{76}$ The obtained results showed that the morphology of Sn particles with different sizes changed simultaneously, whilst the total expanded quantity of irregular-shaped Sn particles was almost identical due to the regions with higher curvatures started first their volume expansion.

To date and to best of our knowledge, there are no representative studies on the use of this technique for characterization of NIBAMs for SIBs and/or PIBs.

\subsubsection{X-ray tomographic microscopy}

This is a powerful and non-destructive technique that allows rapid high resolution quantitative volumetric investigations on diverse samples. Using penetrating X-rays, 3D images of different sections of a sample can be produced by mathematical procedure tomographic reconstruction. This technique provides detailed morphological information on a sample and can be very useful for direct monitoring of the morphological, structural and chemical changes of NIBAMs during degradation. ${ }^{39}$

In 2013, Ebner et al. employed for the first time in operando X-ray tomography to visualize and quantify the origins and evolution of electrochemical and mechanical degradation of $\mathrm{SnO}_{2}$, which was used as NIBAM model, during lithiation processes (Figure 9). ${ }^{84}$ They demonstrated that this technique can provide time-resolved 3D chemical composition and morphology information within individual particles and throughout the electrode. In 2014, Wang and co-workers developed synchrotron X-ray nanotomography for the 3D quantitative analysis and nanoscale visualization of the microstructural evolutions of a Sn based NIBAM during electrochemical cycling in LIBs. ${ }^{85}$ They observed that the most important microstructural changes occurred during the two first cycles, without further significant morphological changes in subsequent series. These finding demonstrates that the initial delithiation-lithiation processes played a key role in the longterm stability and mechanical degradation of this NIBAM.

In 2014, Tariq et al. developed an in-operando X-ray tomography approach to investigate the induced-stress cracking and subsequent delamination during lithiation of a composite $\mathrm{Si}$ based NIBAM. ${ }^{86}$ They demonstrated that this technique was ideal to explore evolutionary processes and quantify them with precision, at real time, and furthermore to study induced failure mechanisms on silicon anodes and in LIBs. In 2017, Vanpeene and co-workers used in-situ synchrotron X-ray computed tomography (XRCT) to investigate the 3D morphological changes of a Si/C paper composite NIBAM during cycling in LIBs. $^{87}$ They quantified the volume fraction, size distribution and connectivity, and also the 3D changes of the NIBAM during operation. They concluded that the Si expansion/contraction due to its volume change was partially irreversible, and it was especially important in both the transverse direction and the bottom part. More recently, in 2019, Tippens et al. used XRCT to reveal the evolution of mechanical damage of Li-metal anodes at the growth interface, in solid-state batteries (SSBs), during electrochemical cycling. ${ }^{88}$ They concluded that the impedance increase and the capacity decay in the SSBs were mainly attributed to the growth of an interface with expanded volume, which fractures the material and its subsequent extent of fracture during cycling. In the same year, Finegan et al. combined high-speed XRD and XRCT to capture temporal descriptions of chemical and morphological heterogeneities (in 3D with high spatial resolution) of silicon-graphite composite NIBAM during lithiation-delithiation processes. ${ }^{89}$ The finding showed that, during lithiation, the shell was constituted of an amalgamation of metastable lithium silicide $\left(\mathrm{Li}_{\mathrm{x}} \mathrm{Si}\right)$ phases, whilst during delithiation, the core acquired a mixed state of $\mathrm{c}-\mathrm{Si}$ and metastable $\mathrm{Lix}_{\mathrm{x}} \mathrm{Si}$. They also observed subparticle lithiation heterogeneities in both $\mathrm{Si}$ and graphite constituents.

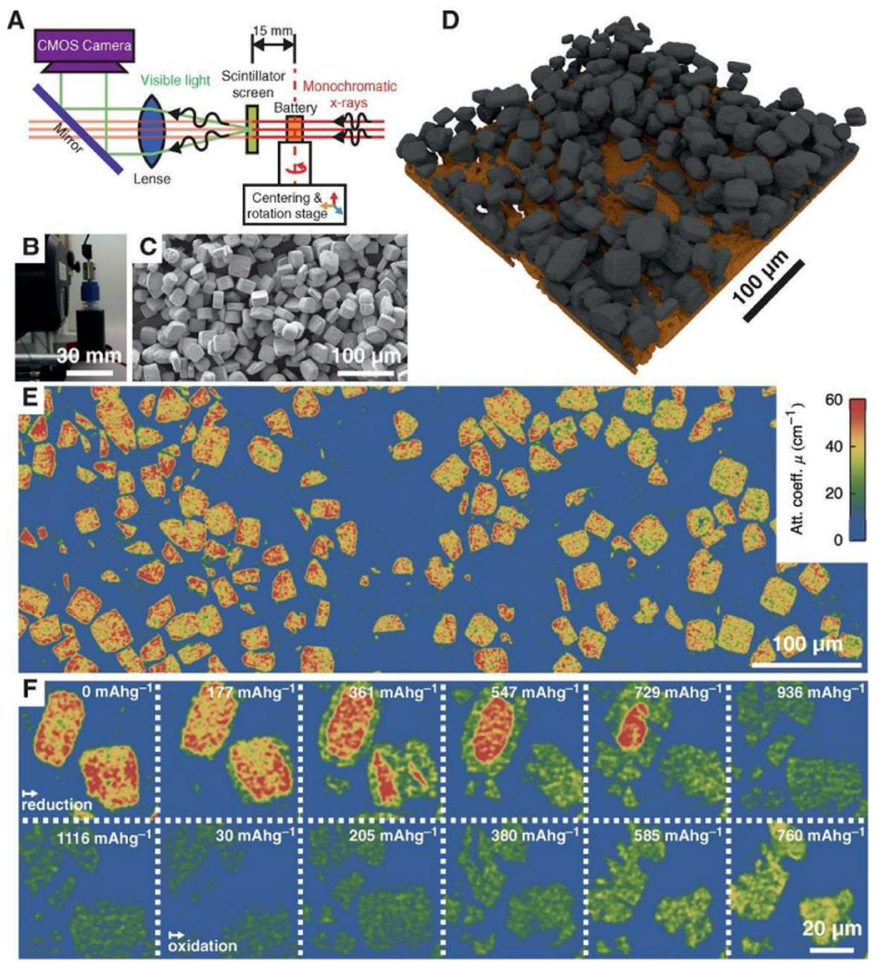

Figure 9. (A) Sketch and (B) photograph of the x-ray tomography setup (CMOS, complementary metal-oxide semiconductor); (C) Scanning electron micrograph of $\mathrm{SnO}$ particles; (D) 3D visualization of x-ray tomograms recorded during battery operation; (E) Unprocessed cross-sectional tomogram showing individual $\mathrm{SnO}$ particles in the electrode with high resolution and good contrast against a low-attenuating carbon black, binder, and electrolyte phase (Att. coeff, attenuation coefficient); (F) A series of cross sections through two particles demonstrates a core-shell process, volume expansion, and particle fracture during the initial reduction and particle redensification during subsequent oxidation $\left(\mathrm{mAhg}^{-1}\right.$, milliampere hours per gram). Reproduced with permission. ${ }^{84}$ Copyright 2013, American Association for the Advancement of Science. 


\subsubsection{X-ray photoelectron microscopy (XPS)}

XPS is a surface-sensitive quantitative spectroscopic technique that measures the elemental composition, chemical and electronic states of the elements within a sample. XPS can detect all the chemical elements with an atomic number greater than or equal to 3 (Li). XPS not only shows the elemental composition but also how they are bonded to. In XPS a sample is irradiated with a beam of X-rays, whilst the kinetic energy and number of escaped electrons are measured (surface analysis depth $\sim 10 \mathrm{~nm}$ ). Although ambient-pressure XPS mode is in development, XPS usually requires high or ultrahigh vacuum conditions

XPS can be very useful to analyse the change of the chemical state of the elements during charge-discharge processes. However, most of the chemical phases relevant for battery interfaces are poor electronic conductors, making it difficult for the phase identification, which is mainly inferred from the absolute XPS core level binding-energies (BEs). For instance, in LIBs many SEI phases, such as $\mathrm{Li}_{2} \mathrm{O}, \mathrm{Li}_{2} \mathrm{O}_{2}$, $\mathrm{LiOH}$, and $\mathrm{Li}_{2} \mathrm{CO}_{3}$, contain both $\mathrm{Li}$ and $\mathrm{O}$, and in some of these phases the Li-to-O ratio is almost identical. In addition, BEs shift during charge process and overlap XPS core levels by the coexistence of multiple Li containing phases in the SEI intensify this obstacle..$^{90}$ Although straightforward approaches for removing charging effects from XPS data sets are being carried out, all these inconveniences make that XPS in operando mode is not one of the most widely used electroanalytical techniques for real time monitoring of NIBAM.

In LIBs, as remarkable study, in 2016, Tang et al. combined in situ XPS with Auger electron spectroscopy to characterize the evolution of bonding and surface chemistry of $\mathrm{CuO}$ NPs NIBAM during cycling. ${ }^{91}$ They showed the elucidation of the changes in $\mathrm{Cu}$ oxidation state during the initial $\mathrm{Li}$ insertion, without the introduction of classical artefacts provided by ex situ measurements. In 2018, combing operando XPS with Auger electron spectroscopy mapping, Wood et al. investigated the formation and evolution of the $\mathrm{Li} / \mathrm{Li}_{2} \mathrm{~S}-$ $\mathrm{P}_{2} \mathrm{~S}_{5}$ SEI on Li-metal anode during cycling, measuring individual overpotentials associated with specific interphase constituents (Figure 10). ${ }^{92}$ More recently, Madec and co-workers used operando XPS with advanced Auger electron spectroscopy to direct evidence the conversion type reactions on TiSnSb-based NIBAM crosssections over long-term cycling. ${ }^{93}$ They highlighted both the role of $\mathrm{Ti}$ as inactive element on the conversion reaction and the excellent long-term stabilitity of the TiSnSb NIBAM after 400 cycles.

\subsubsection{X-ray fluorescence (XRF)}

This technique is based on the detection of secondary X-rays (i.e. emitted or fluorescent) from a sample which was previously excited by high-energy X-rays. For the element of interest, the X-ray energy can be set just above the absorption edge. If the incoming X-ray energy is set above the absorption edge for several elements in a sample, they will all fluoresce at different energies. Therefore, X-ray fluorescence imaging can be advantageous over X-ray absorption for gathering elemental distribution, especially in dilute samples. If the energy is scanned below and through the edge, fluorescence detectors can also be used to produce X-ray absorption spectra in a manner that is compatible with spectromicroscopy. ${ }^{55}$
The first study using operando total reflection XRF was reported in 2001 by Terada et al. to study $\mathrm{Mn}$ dissolution from $\mathrm{LiMn}_{2} \mathrm{O}_{4}$ spinel cathodes in lithium secondary batteries. ${ }^{94}$ In 2012, Robert et al. illustrated the beneficial effects of combining spatially resolved XRF with XRD under operando conditions to detect and correlate the distribution of $\mathrm{Cu}$ and $\mathrm{Mn}$ in layered oxysulfide $\mathrm{Sr}_{2} \mathrm{MnO}_{2} \mathrm{Cu}_{3.5} \mathrm{~S}_{3}$ particles as cathode material..$^{95}$ In 2014, Yamamoto and co-workers used in situ total-reflection XRF to compare the effects of the electronic structure at the electrode/electrolyte interface on the cyclic performance of the $\mathrm{LiCoO}_{2}$ and $\mathrm{LiFePO}_{4}$ thin-film cathode materials. ${ }^{96}$ They concluded that the increased stability of the electronic structure at the $\mathrm{LiFePO}_{4} /$ electrolyte interface affected positively to its cycling performance.

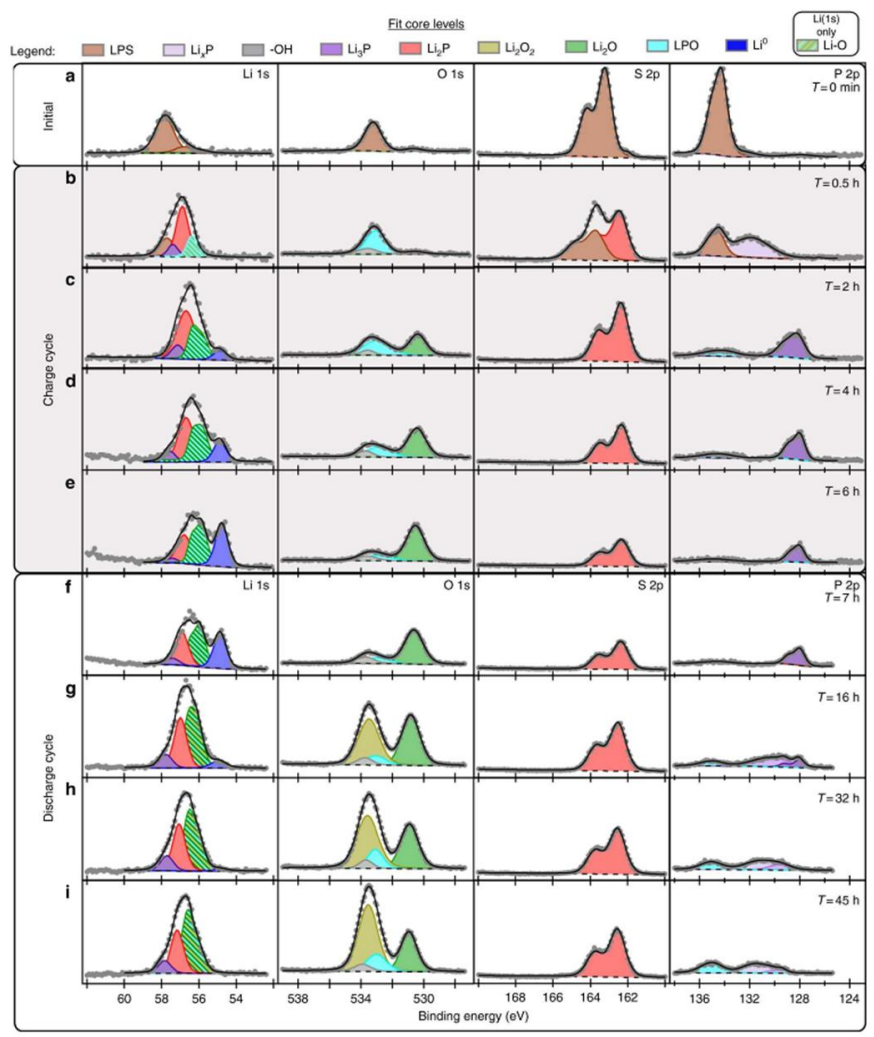

Figure 10. Operando X-ray photoelectron spectroscopy of solid electrolyte interphase formation and evolution in $\mathrm{Li}_{2} \mathrm{~S}-\mathrm{P}_{2} \mathrm{~S}_{5}$ solid-state electrolytes. Reproduced with permission. ${ }^{92}$ Copyright 2018, Springer Nature.

Later, in 2015, Li et al. developed an operando liquid fluorescenceyield X-ray microscopy (FY-XRM) platform, operating in the soft Xray regime, to dynamically follow lithium migration in $\mathrm{LiFePO}_{4}$ cathode electrodes cycling in an organic electrolyte (using Li-metal as anode). ${ }^{97}$ Using two type of particles (ellipsoidal and platelet), they tried to elucidate the Li-intercalation pathway in order to improve the battery performance. They showed a clear dichotomy in the intercalation pathway, i.e. ellipsoidal particles intercalated sequentially and concentrated the current in a small number of particles, whilst platelet ones intercalated simultaneously and guided to a significantly more uniform current distribution. In the same year Boesenberg and co-workers used fast micro-XRF spectroscopy to visualize the effects of the cycling rate and state-of-charge on the elemental distribution ( $\mathrm{Ni}$ and $\mathrm{Mn}$ ) for $\mathrm{LiNi}_{0.5} \mathrm{Mn}_{1.5} \mathrm{O}_{4} /$ carbon composite cathodes in LNMO/Li cells. ${ }^{98}$ They observed significant 
effects on the cathode morphology and elemental distribution, such as the formation of elemental hot-spots and material erosion. More recently, in 2017, Durham et al. combined synchrotron XRD with high-energy XRF nanoprobe mapping, under in situ conditions, to investigate both the lithiation-delithiation mechanisms and the structural evolution of pristine of silver ferrite $\left(\mathrm{AgFeO}_{2}\right)$ and the $\mathrm{Ag}_{0.2} \mathrm{FeO}_{1.6}$ composite in a $\mathrm{Li} / \mathrm{AgFeO}_{2}$ cell. ${ }^{99} \mathrm{XRF}$ was mainly applied to spatially resolve the progression of discharge process (Figure 11). To the best of our knowledge, XRF under operando conditions has not been yet rarely used to the date for the characterization study of NIBAMs in metal-ion batteries.

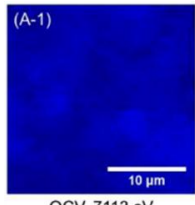

$\mathrm{OCV}, 7113 \mathrm{eV}$

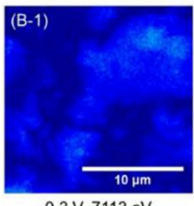

$0.3 \mathrm{~V}, 7113 \mathrm{eV}$

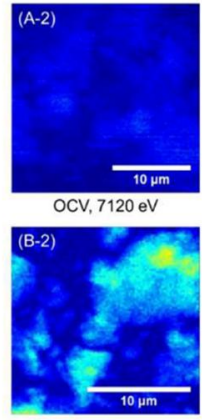

$0.3 \mathrm{~V}, 7120 \mathrm{eV}$

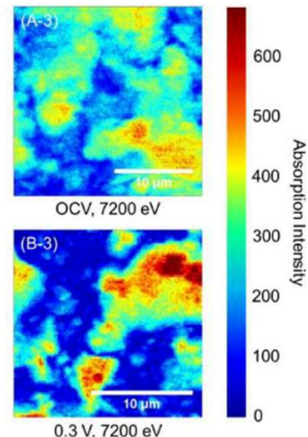

$0.3 \mathrm{~V}, 7200 \mathrm{eV}$
Figure 11. Selected X-ray fluorescence maps at $7113 \mathrm{eV}, 7120 \mathrm{eV}$, and 7200 $\mathrm{eV}$ collected from in-situ cells at open circuit voltage (Cell A) and discharged to $0.3 \mathrm{~V}$ (Cell B). Reproduced with permission. ${ }^{99}$ Copyright 2017, Royal Society of Chemistry.

\subsubsection{X-ray scattering}

$\mathrm{X}$-ray scattering is based on observing the scattered intensity of an Xray beam hitting a crystalline material as a function of incident and scattered angle, polarization, and wavelength or energy. This is a nondestructive electroanalytical technique that provides information about the crystal structure, chemical composition, and physical properties of battery materials under synthesis and operational conditions. ${ }^{100}$

In 2015, Silberstein et al. used operando X-ray scattering, XRD and XAS to determine the structural and bonding changes of Ge-NW NIBAM in LIBs, as a function of the lithium content and state of charge. ${ }^{101}$ They observed that lithium incorporation within the Ge nanostructure took place heterogeneously, preferentially into amorphous regions over crystalline domains. In 2017, Bhaway and co-workers combined in situ grazing-incidence small-angle X-ray scattering and X-ray diffraction (GISAXS/GIXD) to assess the structural evolution of a family of model ordered mesoporous $\mathrm{NiCo}_{2} \mathrm{O}_{4}$ films as NIBAM during battery operation. ${ }^{102}$ They showed that the pore sizes affected the stability of the ordered nanostructure during the cycling, observing a failure of the ordered nanostructure with small mesopores $(\approx 9 \mathrm{~nm})$ during the first two charge-discharge cycles. They demonstrated that these operando measurements provided insight into how changes at the atomic scale were transferred to the nanostructure during battery operation. Later, in 2018, Möhl and co-workers used operando small-angle X-ray scattering (SAXS) to investigate the morphological evolution of a nanostructured polymer electrolyte for LIBs. ${ }^{103}$ They showed that the block copolymer electrolyte seemed morphologically more favourable for the cell performance, and it was independent of the choice of the NIBAM material due to better electrode/electrolyte compatibility.
And more recently, in 2019, Berhaut et al. employed simultaneous operando synchrotron wide-angle (WAXS) and SAXS to investigate multiscale multiphase lithiation-delithiation mechanisms in an a-Si/c$\mathrm{FeSi}_{2} /$ graphite composite NIBAM after prolonged cycling. ${ }^{104}$ They showed that, simultaneously and independently, WAXS supplied the information of the local crystalline structure, whilst SAXS provided information on the silicon state of (de)lithiation and nanoscale morphology. They concluded that the SAXS-WAXS combination provided a complete picture of the C-rate-dependent sequential (de)lithiation mechanism of this composite NIBAM under operation conditions.

\subsection{Electron microscopy}

Electron microscopy uses a beam of accelerated electrons, as a source of illumination, providing very short wavelengths and obtaining high resolution images. There are different electron microscopy techniques, such as Transmission electron microscopy (TEM), Scanning electron microscopy (SEM) and Electron holography (EH), which can provide a combination of nanoscale down to atomic level spatial resolution and millisecond temporal resolution that is not achieved by any other technique.

\subsubsection{Transmission electron microscopy (TEM)}

In TEM a beam of electrons is transmitted through a sample supported on a grid to form an image. This microscopy technique provides much higher resolution than light microscopes, owing to the smaller de Broglie wavelength of electrons. An enormous number of operating modes are possible by TEM, such as conventional imaging, scanning TEM imaging (STEM), diffraction, spectroscopy, and their combinations. Overall, TEM reveals not only morphological features of NIBAMs but also accuracy information about its structure, chemical composition and uniformity. ${ }^{105}$

For LIBs, at the beginning, in situ TEM studies used an open-cell configuration, which provided fundamental real-time insights into the structural, morphological and chemical evolution of the NIBAMs during lithiation. Greatly, in 2011, Liu et al. created the first nanobattery inside a TEM for allowing real time and atomic scale observations of battery cycling. ${ }^{105}$ Two types of nanobattery cells were proposed, one based on room temperature ionic liquid electrolytes (ILEs) and the other based on all solid components. Using ILE cell-based, they performed in situ TEM analysis of different NIBAMs, such as $\mathrm{SnO}_{2} \mathrm{NWs},<112>$ oriented Si NWs, carbon coated $\mathrm{SnO}_{2} \mathrm{NWs}$, and individual Si NPs. They demonstrated that in situ TEM was a powerful tool to investigate key parameters which modulates the electrochemical behaviour of NIBAMs such as size and crystallographic orientation. In 2013, McDowell et al. applied this technique to compare the lithiation/delithiation process of a-Si and cSi nanospheres. ${ }^{106}$ They concluded that, for the use in LIBs, a-Si spheres had more favourable lithiation kinetics and fracture behaviour than c-Si ones (e. g. $870 \mathrm{~nm}$ a-Si spheres did not fracture upon lithiation that was much larger size than the critical fracture diameter of c-Si ones, $150 \mathrm{~nm}$ ). Later, using the same type of NIBAM, Gu and co-workers developed an operando TEM electrochemical liquid cell, which provided a more realistic configuration in comparison with real batteries, allowing the use of relevant liquid electrolytes (i.e. it was 
not limited to solid lithium oxide or an ionic liquid ones). ${ }^{107}$ They demonstrated that this configuration provided quantitative information about the SEI layer formation and its structural and chemical evolution.

TEM technique not only can be applied for silicon-based NIBAM but also for different types. For instance, in 2015, Xu and co-workers employed in situ TEM to provide direct insights of the evolution and conversion reactions onto a composite NIBAM which was composed of $\mathrm{Fe}_{3} \mathrm{O}_{4}$ NPs uniformly dispersed in carbon nanofiber (CNF) matrix. ${ }^{108}$ They observed that, upon initial lithiation, the $\mathrm{Fe}_{3} \mathrm{O}_{4} \mathrm{NPs}$ in the CNF matrix were gradually reduced to $\mathrm{Fe}$ nanograins along the $\mathrm{Li}$ ion diffusion direction, whilst after delithiation $\mathrm{FeO}$ emerged as new oxidation product. In 2016, Su et al. used in situ TEM to investigate the fundamental lithiation/delithiation mechanism of $\mathrm{ZnFe}_{2} \mathrm{O}_{4} \mathrm{NPs}$, a type of mixed transition-metal oxides (MTMOs). ${ }^{109}$ In this case, they observed that during the first lithiation the $\mathrm{ZnFe}_{2} \mathrm{O}_{4}$ NPs generated a composite structure of $\mathrm{Fe}$ and $\mathrm{Zn}$ nanograins within $\mathrm{Li}_{2} \mathrm{O}$ matrix, whilst during the delithiation the complete conversion to $\mathrm{Fe}_{2} \mathrm{O}_{3}$ and $\mathrm{ZnO}$ took place. They demonstrated that the finding can be useful to optimize the composition of this type of NIBAM for the improvement of its electrochemical performance.

On the other hand, High-resolution TEMs (HR-TEMs) can provide real-time and atomic-scale tracking of fully reversible lithium insertion and extraction processes in NIBAMs (i.e. with a resolution of $0.08 \mathrm{~nm}$ ). HR-TEM is a suitable in situ technique for understanding the functional and failure mechanisms of these anodes. For instance, Gao and co-workers used HR-TEM to investigate the reversible phase transitions in $\mathrm{SnS}_{2}$ nanostructures during lithiation and delithiation cycles. ${ }^{110}$ The finding revealed the dynamics and kinetics of lithium insertion and extraction in the van der Waals interactions dominated $\mathrm{SnS}_{2}$, inferring insights to improve the energy efficiency of LIBs. In 2017, He and co-workers used this operando technique to study the structural, morphological, and chemical evolutions of ultrathin hexagonal copper sulfide (CuS) nanoflakes during charge-discharge cycling. ${ }^{111}$ They reported evidences of the dynamical morphological and structural evolution during lithiation, both in plan-view and in side-view, by in situ annular dark-field (ADF)-STEM mode (Figure 12). The difference in the scattering of the electrons between different regions or phases gives rise to contrast by ADF-STEM. For this, a focused electron beam is scanned across the sample, and the transmitted electrons scattered from each point of the raster are recorded by an annular detector to form a dark-field STEM image. ${ }^{112}$ The obtained results indicated a two-step lithiation pathway through $\mathrm{Li}$ intercalation and $\mathrm{Cu}$ extrusion.

Whilst for SIBs, in situ TEM has been recently used by Huang et al. to understand the structure and phase transitions of few-layer bismuth nanosheets (NSs) during sodiation/desodiation processes. ${ }^{113}$ Comparing to the lithiation process, the sodiation one on metal sulfides may suffer different mechanism, depending on the metal and structural type. In situ HR-TEM imaging was successful used to identify the multistep phase transition stages from $\mathrm{Bi} \rightarrow \mathrm{NaBi} \rightarrow \mathrm{c}-$ $\mathrm{Na}_{3} \mathrm{Bi}$ (cubic) $\rightarrow$ h-Na $\mathrm{Bi}$ (hexagonal). Finally, the good structural stability of this Bi based NIBAM after sodiation/desodiation cycles was demonstrated. Wang et al. also used this technique to investigate the sodiation mechanism of $\mathrm{TiS}_{2}$ nanoflakes. ${ }^{114}$ They observed multiple intermediate phase transitions, which were different from its lithiation counterpart, with varied sodium occupation sites and interlayer stacking sequences. They suggested that the reversible $\mathrm{Na}$ accommodation and extraction into the $\mathrm{TiS}_{2}$ NIBAM was mainly due to its weak interlayer van der Waals interactions.
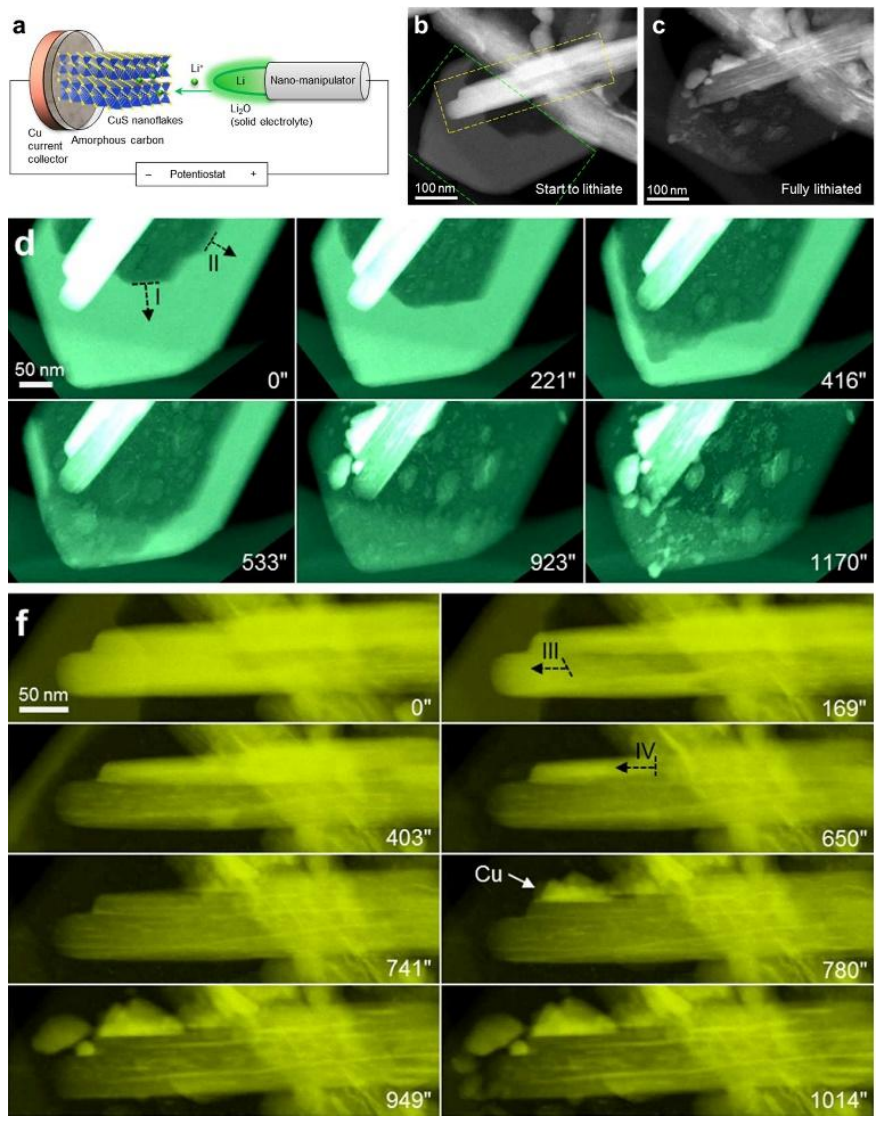

Figure 12. (a) Schematic representation showing in situ battery setup for STEM observation. STEM images of CuS nanoflakes before (b) and after (c) lithiation. Time sequential STEM images during in situ lithiation showing reaction in plan-view (d) and side-view (e), corresponding to the areas of green and yellow dashed boxes in (b), respectively. Reproduced with permission. ${ }^{111}$ Copyright 2017, American Chemical Society.

\subsubsection{Scanning electron microscopy (SEM)}

SEM produces images of samples on a conductive support by scanning their surfaces with a focused beam of electrons. These subatomic particles interact with atoms in the sample, providing characteristic signals collected by an Everhart-Thornley detector about its composition and surface morphology. Conventional SEM achieves resolution better than $1 \mathrm{~nm}$ but requires high vacuum conditions, which is an important drawback for the use in real-time analysis of NIBAMs (i.e. limited to non-volatile electrolytes).

For LIBs, Chen et al. used in situ SEM to study the lithiation/delithiation behaviour of silicon based NIBAMs with different sizes and shapes (i.e. microparticles, nanoparticle aggregates, and thin flakes). ${ }^{115}$ They demonstrated that the surface structural changes of these NIBAMs during cycles were strongly dependent on their particle-size and shape. They concluded that operando SEM can simultaneously provide useful information about the morphological variations and elemental distributions of NIBAMs 
during charge-discharge cycles. Later, this same research group investigated the morphological changes on a polyimide-binder Simicroparticle NIBAM during the lithiation-delithiation processes, using a specially modified coin cell for in situ SEM. ${ }^{116}$ Although this approach was only applicable for non-volatile electrolytes, it provides visual information about the interaction between the $\mathrm{Si}$ active material, the binder, and the electrolyte. They concluded that the free space on the anode was a critical factor in enhancing the electrochemical properties of this type of NIBAM. Recently, there is a growing interest for mass production of Li-based anodes, which can be used in high energy density batteries. ${ }^{117}$ Nevertheless, the formation of a robust and stable SEI layer is crucial to protect the $\mathrm{Li}$ metal surface and suppress the detrimental dendritic growth. In this direction, Hwang et al. used SEM analysis at various current densities to investigate the effect of the $\mathrm{LiDFOB}$ additive and $\mathrm{LiNO}_{3}$ treated Li-based anode. ${ }^{118}$ After assessment the morphology of the deposited Li metal using coin-type $\mathrm{Li} / \mathrm{Li}$ half-cells, they concluded that, as the current density increases, the deposited $\mathrm{Li}$ particle became progressively smaller and elongated. The different Li morphologies observed by SEM together with the Sand's time relationship also allowed the estimation of the dendrite nucleation for the electrolytes.

On the other hand, Zhou and co-workers used a focused-ion beamscanning electron microscope (FIB-SEM) approach, which was previously developed by Miller et al. for the real-time monitoring of cathodes in LIBs, ${ }^{119}$ to monitor the microstructural evolution of a single Sn microparticle during cycling. ${ }^{120}$ The FIB-SEM setup uses a focused beam of ions instead of a beam of electrons. The obtained results showed clear evidences of the mechanical degradation and deformation mechanism of this type of NIBAM during the LIBs electrochemical reactions (i.e. formation and evolution of cracks and porous structures at different states of charge) (Figure 13).

\subsubsection{Electron Holography (EH)}

$\mathrm{EH}$ is an electron-microscopy-based interferometric technique, where the sample is exposed to an electron beam of which the phase will be modulated by the electrostatic potential and magnetic field across the samples. The signal coming from the sample and a reference signal are let to interfere with each other, which results in a hologram, i.e. an interference fringe pattern. The phase modulation extracted from the hologram is used to reconstruct the electrostatic and/or magnetic fields in the samples, e.g. the inner mean potential. In summary, EH requires

(a)
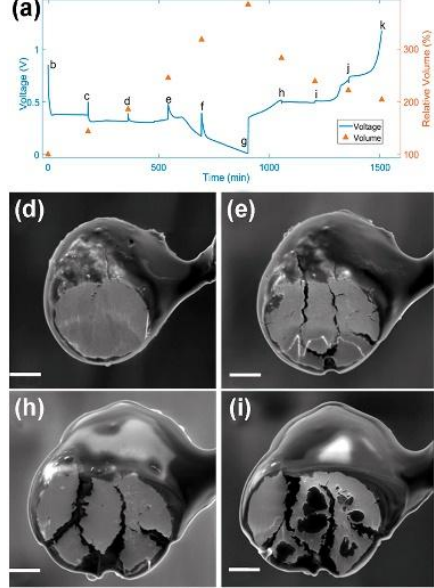

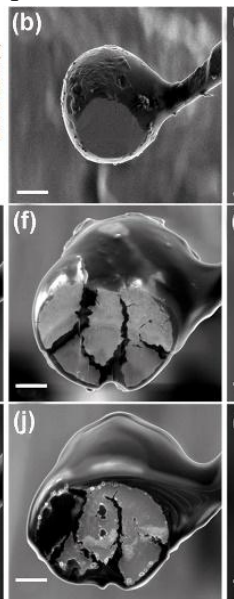

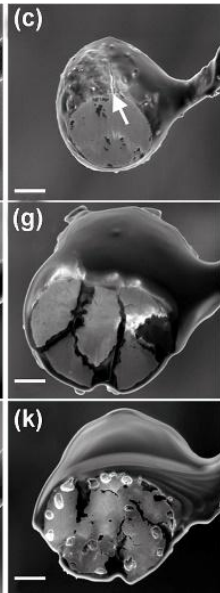

two imaging processes to display the electromagnetic fields. An excellent schematic representation of the EH principle can be observed in Figure 3 from the paper reported by Yamamoto et al. ${ }^{121}$

Figure 13. Sn particle fully discharged/charged at $0.3 \mathrm{nA}$. (a) Voltage profile and relative volume of the first cycle. (b) SEM image of as-prepared tin particle. (c-g) SEM images of the same particle at $20,40,60,77$, and $100 \%$ of discharge states, respectively. (h-k) SEM images at 25, 50, 75, and $100 \%$ of charge states, respectively. Scale bar is $3 \mu \mathrm{m}$. Reproduced with permission. ${ }^{120}$ Copyright 2019, American Chemical Society.

Gan et al. have used off-axis electron holography to measure charge distribution during lithium ion insertion into a Ge nanowire (NW) under dynamic operating conditions. They discovered that the surface region of the Ge core is negatively charged during the core-shell lithiation of the Ge NW, which is counterbalanced by positive charge on the inner surface of the lithiated $\mathrm{Li}_{\mathrm{x}} \mathrm{Ge}$ shell (Figure 14). The remainder of the lithiated $\mathrm{Li}_{x} \mathrm{Ge}$ shell is free from net charge, consistent with its metallic characteristics. The work provided a vivid picture of charge distribution and dynamic evolution during Ge NW lithiation, and should form the basis for tackling the response of these and related materials under real electrochemical conditions. ${ }^{122}$ Recently, Wen et al. used in-situ EH to study the lithiation mechanism of $\mathrm{Li}_{4} \mathrm{Ti}_{5} \mathrm{O}_{12}$ (LTO), they found that double charge layers are formed at the interface of the insulating LTO (Li4) phase and the semiconducting $\mathrm{Li}_{7} \mathrm{Ti}_{5} \mathrm{O}_{12}$ ( $\mathrm{Li7}$ ) phase, and can greatly boost the lithiation kinetics. The electron wave phase of the LTO particle is found to gradually shrink with the interface movement, leaving a positive electric field from Li7 to Li4 phase. Once the capacitive interface charges are formed, the lithiation of the core/shell particle could be established within 10s. The ultrafast kinetics is attributed to the built-in interface potential and the mixed $\mathrm{Ti}^{3+} / \mathrm{Ti}^{4+}$ sites at the interface that could be maximally lowering the thermodynamic barrier for Li ion migration. ${ }^{123}$
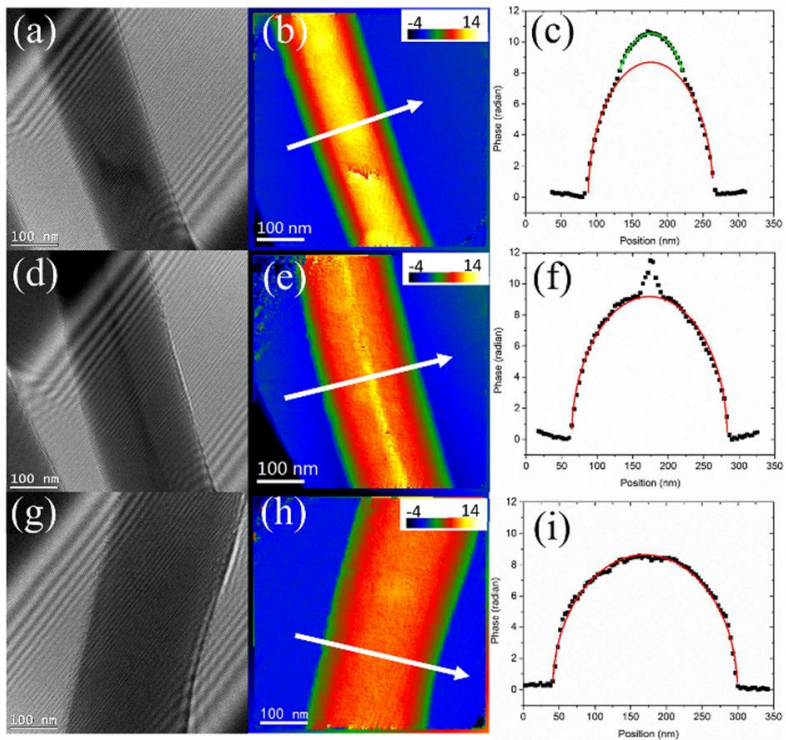

Figure 14. Electron holography observations of Ge/Li x Ge core/shell NW during lithiation: (a), (d) and (g) Holograms of NW; (b), (e) and (h) Corresponding reconstructed phase images, shown in pseudo-color (scale bar shown at top right in units of radian); (c), (f) and (i) Phase profiles along the white arrows in (b), (e) and (h), respectively. Reproduced with permission. ${ }^{122}$ Copyright 2016, American Chemical Society. 


\subsection{Scanning probe microscopy}

Scanning probe microscopy (SPM) is a branch of the microscopy that creates images of a sample by scanning its surface with a physical probe. SPM can monitor the interfacial topography/morphology and electrochemical properties at nanoscale resolution. There are different modes varying the manner of using these interactions to obtain an image, such as atomic force microscopy (AFM), scanning electrochemical microscopy (SECM), scanning ion conductance microscopy (SICM), electrochemical strain microscopy (ESM), etc.

\subsubsection{Atomic Force Microscopy (AFM)}

AFM is a scanning probe microscopy technique which provides information by touching the surface with a mechanical probe (i.e. by a cantilever tip). There are at least three different modalities of AFM: Non-contact (force measurement), tapping (imaging), and lithography (surface manipulation) modes. Compared with TEM, in situ AFM can provide information about the interfacial structure of NIBAMs and its topography evolution with the SEI layer formation during cycles. The major limitation is the technical difficulties to allow the access of the AFM cantilever into the battery cell whilst maintain the inert atmosphere required for the electrochemical measurements.

Recently, in LIBs, Breitung et al. used in situ AFM to analyse the surface topography of a high-capacity nano-silicon NIBAM during charge-discharge cycles, containing a polymer binder and a carbon black additive. ${ }^{124}$ This work showed that AFM provided high resolution images at real-time about the changes in particle size as well as the formation and evolution of the SEI thickness. They concluded that this was a powerful technique which could be applied to important battery materials without restriction to thin film geometries. Similarly, Yoon and co-workers measured by in situ AFM both the $\mathrm{Si}$ expansion ratio and the charge capacity versus the equilibrium lithiation/delithiation potential, in order to investigate the influence of electrolyte composition on SEI evolution. ${ }^{125}$ They demonstrated that these in situ measurements allowed real-time monitoring of the SEI layer growth and the volume changes of these thin film a-Si NIBAMs.

In the case of SIBs, Lacey et al. used in situ AFM to investigate the intercalation model on NIBAMs composed of mechanically exfoliated molybdenum disulfide $\left(\mathrm{MoS}_{2}\right)$ flakes. ${ }^{126}$ This operando technique provided the quantitative data on the SEI thickness into $\mathrm{MoS}_{2}$ electrodes, showing the evidences of their structural changes at the nanoscale. On the other hand, Han et al. reported an operando AFM study of the sodiation and desodiation on Sn NPs with different sizes which were used as probing NIBAMs. ${ }^{127}$ The measurements were performed in an aprotic liquid electrolyte akin to reproduce the environment of SIBs cells. They concluded that, after desodiation, the smaller Sn NPs showed a better shape maintenance but a lower rate of irreversible volume change than the larger ones.

To the date, no bibliography has been found on the use in situ AFM for NIBAMs in PIBs.

\subsubsection{Scanning Electrochemical Microscopy (SECM)}

SECM provides spatially resolved information about redox activities, thus it has been employed in electrocatalysis, corrosion, elucidation of charge transfer kinetics and mechanisms. ${ }^{128}$ Recently, SECM was successfully employed in the field of batteries, demonstrating to be a unique and powerful analytical tool for the investigation of several processes occurring at battery electrodes. ${ }^{129}$ Several SECM modes of operation have been developed, including the feedback (positive and negative) mode, the alternating current mode, the tip generation/substrate collection (TG/SC) mode, the substrate generation/tip collection mode (SG/TC), the penetration mode, and the ion transfer mode.

Zampardi et al. applied the feedback mode of SECM to monitor SEI formation on porous $\mathrm{TiO}_{2}$ electrodes used in Li-ion batteries. ${ }^{130}$ They demonstrated SEI formation starting at potentials as high as $1.3 \mathrm{~V}$ vs. $\mathrm{Li} / \mathrm{Li}^{+}$on anatase $\mathrm{TiO}_{2}$ nanoparticles, and observed a significant increase in the feedback current upon intercalation of $\mathrm{Li}^{+}$in $\mathrm{TiO}_{2}$. Later, they further investigated the kinetics of SEI formation on the surface of titania. ${ }^{131}$ Recently, FB-SECM and ac-SECM were applied for in situ visualization of SEI evolution and stability on the $\mathrm{TiO}_{2}$ anode in a concentrated aqueous during cycling. They found that the overall feedback current first increases before becoming relatively stable with cycling, which demonstrates that the conductivity of the substrate, with the SEI-covered and active material exposed areas, increases with the number of cycles due to $\mathrm{Ti}^{3+}$ and Li residuals. The scattered distribution of SEI components on $\mathrm{TiO}_{2}$ surface is composed of $\mathrm{LiF}, \mathrm{Li}_{2} \mathrm{CO}_{3}$, and $\mathrm{Li}_{2} \mathrm{O}$ formed during cycling. For the resting period after SEI formation, an inverse process was observed. Such SEI dissolution with resting time and the stabilization of the substrate toward the equilibrium condition with decreasing conductivity could be understood from the change in the area scan, substrate OCV, and EIS measurement (Figure 15). ${ }^{132}$
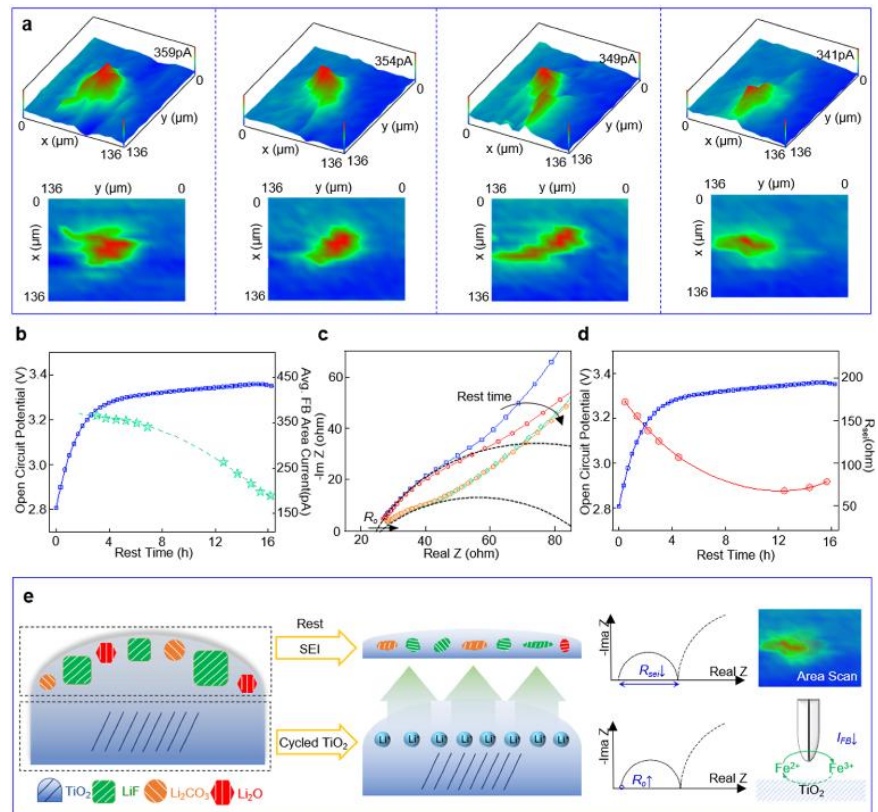

Figure 15. (a) SECM feedback image of an identical region of cycled $\mathrm{TiO}_{2}$ after 3-6 h. (b) Change of OCV and average area feedback current density with the rest time. (c) Nyquist plots of the cycled $\mathrm{TiO}_{2}$ with the rest time; the intercept of impedance plot with real axis is Ro. (d) Change of OCV and the interface resistance R Sei with the rest time. (e) Schematic illustration of the 
interface structure and its evolution with the rest time. Reproduced with permission. ${ }^{132}$ Copyright 2019, American Chemical Society.

\subsubsection{Scanning Ion Conductance Microscopy (SICM)}

SICM is a non-contact technique for nanoscale characterization in aqueous electrolytes which can provide distinct advantages for topographical imaging and electrochemical capabilities of battery electrode at the nanoscale. ${ }^{133}$ The probe of this technique is made of an electrolyte-filled nanocapillary of diameter $50-100 \mathrm{~nm}$. The resolution of SICM depends on the tip geometry and sample-tip distance. Typically, the vertical resolution of SICM is $10 \mathrm{~nm}$ and the lateral resolution is about $50 \mathrm{~nm}$. A resolution of 3-6 nm can be obtained by using a $13 \mathrm{~mm}$ diameter pipette. Generally, the resolution is 3 times the inner diameter of the pipette. ${ }^{134}$ Smaller pipettes have been achieved with quartz, but the increased resistance reduces the signal-to-noise ratio of the ionic current. Nevertheless, the current sensitivity of SICM can reach sub-pA levels, implying that it is wellsuited for the characterization of subtle electrochemical phenomena at the nanometer scale. ${ }^{135}$

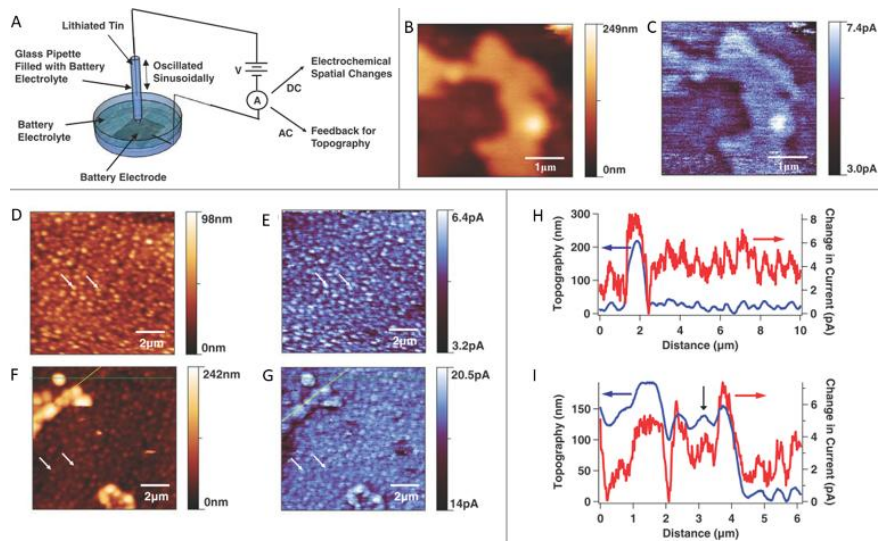

Figure 16. (A) Schematic diagram of the in situ Li-ion battery SICM configuration. The entire apparatus is housed inside of an argon filled glovebox, which permits the use of electrolytes that are employed in actual Liion batteries. SICM (B) topography image and (C) DC current map of silicon nanoparticles and PVDF deposited via drop-casting on copper foil. SICM (D, F) topography and $(E, G)$ DC current images of a $60 \mathrm{~nm}$ thick tin thin film deposited on a $60 \mathrm{~nm}$ thick copper thin film on glass $(\mathrm{D}, \mathrm{E})$ before lithiation and $(\mathrm{F}, \mathrm{G})$ after $24 \mu \mathrm{Ah} \mathrm{cm}^{-2}$ lithiation. Extracted profiles averaged over 8 pixels wide from Figures $\mathrm{F}$ and $\mathrm{G}$ for the $(\mathrm{H})$ green line and $(\mathrm{I})$ yellow line. Reproduced with permission ${ }^{136}$. Copyright 2018, American Chemical Society.

Lipson et al. modified SICM and demonstrated that it is an effective in situ technique for studying both the topography and electrochemistry of battery electrode materials at the nanoscale (Figure 16A). Correlated topographic and current mapping of test structures reveals a number of factors that lead to spatial inhomogeneities including the thickness of the active battery material and barriers to lithiation such as the evolution of the SEI (Figure 16B, C). Subsequent measurements on tin electrodes provide a nanoscale picture of catalytic decomposition of the electrolyte where an SEI is observed to form around electrode regions that rapidly grow during lithiation (Figure 16D-G). In addition, the lithiation process can induce local film growth to the point where further electrolyte decomposition is suppressed (Figure $\mathbf{1 6 H}$, I). It should be noted that SICM is compatible with temporal and voltage spectroscopies, suggesting further possibilities for this technique. Overall, their work established the unique capabilities of SICM as an in situ tool for probing spatial inhomogeneities in topography and electrochemistry in battery electrodes, thus providing new characterization data that can inform ongoing efforts to understand and improve the capacity, lifetime, and safety of lithium ion battery technology. ${ }^{136}$ They demonstrated ultrathin $\mathrm{Al}_{2} \mathrm{O}_{3}$ film on $\mathrm{MnO}$ electrode by atomic layer deposition (ALD) can impact the formation of SEI, and further improve the cyclability performance. ${ }^{137}$

\subsubsection{Electrochemical Strain Microscopy (ESM)}

ESM is a novel scanning probe microscopy technique with unprecedented resolution that emerged in recent years. ${ }^{138}$ The ESM utilizes the strong coupling between ionic concentration and strains in ionic conductors, to deduce the information on ionic flow within the materials, either due to electrode polarization or surface electrochemical processes. This approach allows effectively separating ionic and electronic currents, and hence allows highveracity measurements of the former. The main advantage of this technique is the ability to monitor the electrochemical phenomena in solids at the nanometer scale by measuring dynamic strain, rather than current. $^{139}$

Bake et al. extended ESM to time-voltage spectroscopy for the spatially resolved mapping of Li-ion dynamics in Si-anode materials. They investigated the electrical bias driven Li-ion motion in silicon anode materials in thin film battery heterostructures. ${ }^{138}$ ESM utilizes the intrinsic link between bias-controlled Li-ion concentration and molar volume of electrode materials, providing the capability for studies on the sub-20 nm scale, and allows the relationship between Li-ion flow and microstructure to be established. Thus, the evolution of Li-ion transport during the battery charging is directly observed. Later, the same group further demonstrated that Li-ion flow mediated coupling between bias and strain allows mapping of local mobility and electrochemical reactivity in $\mathrm{Si}$ anode material in battery devices. ${ }^{140}$ They directly quantitatively measured and spatially resolved mapped diffusion times in nanometer scale volumes, exceeding the detection limits of classical electrochemical methods by 6-8 orders of magnitude. ${ }^{141}$

\subsection{Optical techniques}

Optical techniques are particularly useful for the morphological analysis of NIBAMs. They are non-invasive, non-destructive, and non-vacuum analysis, and less restrictive on the design of in situ and/or operando cells. Depending on the length and class of light source, they can be classified as follows: Raman spectroscopy, Fourier transform infrared spectroscopy (FTIR), optical microscopy, multi-beam optical stress sensor, valence-differential absorption spectroscopy, and nonlinear coherent vibrational spectroscopy.

\subsubsection{Raman spectroscopy}

Raman spectroscopy is a powerful characterization technique commonly used to study the relationship between structural and electrochemical data during electrode cycling. The information is obtained through vibrational, rotational and low-frequency modes of the molecules, which are dependent of its crystal symmetry, bond or 
structural (dis)order, and strain. For this, a source of monochromatic light must interact with molecular vibrations, phonons or other excitations whilst the shift in energy is measured. Although Raman spectroscopy typically refers to vibrational mode, there are many other modalities, such as surface-enhanced (SERS), resonance, tipenhanced, polarized, stimulated, transmission, spatially offset, and hyper Raman mode.

Raman spectroscopy is very useful to investigate lithiation mechanisms and the nature of electrode/electrolyte interfaces during cycles (i.e. SEI formation, anode stress, etc.). The major limitation is the difficultly to interpret the resulting spectra. The development of confocal Raman microscopy has been an important advance on this research field, allowing concurrent analysis of both the electrodes and electrolyte by measuring a specified spot of a sample and obtaining a mapping image. ${ }^{142}$ An extensive review focused on the use of in situ Raman for the analysis of electrode materials in LIBs has been recently reported by Julien et al.. ${ }^{143}$

Some remarkable examples of research works using this technique for investigate different types of NIBAMs in LIBs are introduced below. In 2016, Zheng et al. reported the stress induced by the lithiation in nanostructured Si anodes using micro-Raman spectroscopy mode. ${ }^{144}$ They concluded that tensile stress in the a-Si/Lix layer of the SiNPs explained the cracks developed during cycles. One year later, Jana and co-workers studied the evolution of the stress generated in a single cSi during lithiation which was the main cause of failure and fracture of these NIBAMs. ${ }^{145}$ They demonstrated that in situ Raman was an ideal tool to understand the progressive failure mechanism of single c-Si anodes. Whilst Cabo-Fernandez and co-workers used Raman to investigate the (de)lithiation mechanism on carbon-coated $\mathrm{ZnFe}_{2} \mathrm{O}_{4}$ (ZFO) anode ${ }^{146}$ which is a TMO-based NIBAM type. The resulting Raman spectra showed that the lithiation occurred both in ZFO nanoparticles and in the carbon coating, and a clear increment of the disorder of the coating after the first (de)lithiation process.

Despite of all efforts made to date, in situ Raman spectroscopy needs to be combined with others operando techniques to further understand phenomenon that occurring. One of the most promising in situ combination is Raman plus XRD because the latter technique provides complementary information about the structural evolution and phase transformation of the anode materials, whilst the former one measures the stress and strain as well as the local disorder. For LIBs, an excellent example is the work reported by Tardif et al. (Figure 17). ${ }^{147}$ They investigated the internal structure of NIBAMs based on silicon nanoparticle during two discharge/charge cycles by analysing the intensity and position of Si diffraction peaks and Raman phonons. They showed that different lithiation processes occurred in the first and the second cycle. More recently, Krause et al. reported this in situ combination for studying the lithiation and delithiation of uncoated and carbon-coated silicon nanowires. ${ }^{148}$ The provided information about the different species in the battery cell, allowing to explain the relationship between the resulting structural and electrochemical data.

For SIBs, an example of this same combination was reported by Li el al., ${ }^{149}$ who obtained insights into the structural evolution and sodiation dynamics by investigating the ether-induced interfacial electrochemical characteristics of anatase $\mathrm{TiO}_{2}$ coupled with a diglyme-based electrolyte. They concluded that the use of a diglymebased electrolyte could improve the electrochemical performance of the different anodes for SIBs.
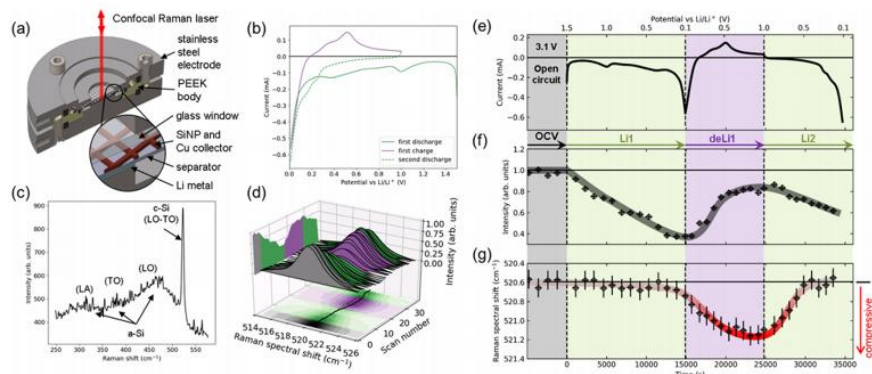

Figure 17. (a) Experimental operando Raman setup and electrochemical cell. (b) Linear sweep cyclic voltammograms. (c) Typical Raman spectra including crystalline and amorphous silicon peaks. (d) Evolution of the c-Si TO-LO peak as a function of the scan number during the open-circuit (OCV), discharge (green), and charge (purple) cycles. The shadow on the left panel shows the variation of the Raman peak intensity over cycling time, and the intensity map on the lower panel indicates the shift of the peak position (highlighted by the black line). Evolution of the (e) current, (f) Raman peak intensity, and (g) spectral position as a function of time and applied voltage. Dashed lines indicate the end of the (de)lithiation processes. Reproduced with permission. ${ }^{147}$ Copyright 2017, American Chemical Society.

\subsubsection{Fourier transform infrared (FTIR) spectroscopy}

A priori, in situ infrared spectroscopy would be an ideal method for real-time monitoring the chemical modification of the NIBAMs surface into the metal-ion batteries during the charge-discharge process, mainly due to its fast data acquisition and high molecular specificity. This spectroscopic technique is valid to identify the presence of characteristic functional groups and chemical bonds both for organic and inorganic materials. ${ }^{8}$ Despite that this technique provides qualitative and quantitative information, it has at least two major inconveniences that must be considered in order to design an appropriate cell battery for in situ FTIR measurements. Firstly, the absorption of the electrolyte implies the use of thin-layer external reflectance or internal reflectance techniques. Secondly, sensitivity problems due not only to the low absorption from the generated species but also to high signal of the previously present ones, which requires subtraction of the spectra recorded at different potentials and/or times.

An excellent example of in situ FTIR analysis for LIBs was reported by Shi et al., using attenuated total reflection (ATR) mode, to analyse the SEI formation on a silicon based-NIBAM under different surface conditions and potentials. ${ }^{150}$ The obtained results demonstrated that the loss of capacity and the passivation with the successive cycles of this NIBAM were attributed to the formation of decomposition products from diethyl carbonate (an electrolyte component) on the Sioxide layer (i.e. SEI layer). This operando ATR-FTIR spectroscopy was also applied to study the dynamic interfacial properties of $\mathrm{NiSb}_{2}$, a representative alloy-based NIBAM for LIBs, during cycling and in the presence of a commercial electrolyte $\left(\mathrm{LiPF}_{6}\right)$ dissolved in a mixture of additives, such as ethylene carbonate (EC) and dimethyl carbonate (DMC). ${ }^{151}$ They demonstrated that this technique allowed the real-time monitoring of the lithium content in the electrolyte solution during cycling. 


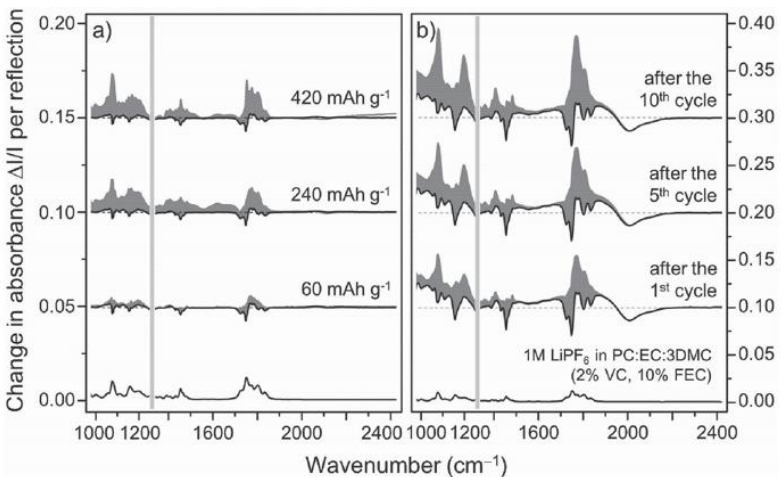

Figure 18. Infrared spectra of the reconstructed Solid/Electrolyte interphase, after correcting for the electrolyte-displacement effects, calculated a) at several steps of the first lithiation and b) along cycle life. Black lines are the measured spectra and the gray areas represent the reconstructed spectra. Reproduced with permission. ${ }^{152}$ Copyright 2016 , Wiley.

An important enhance in sensitivity of in situ FTIR was obtained by Dalla Corte and co-workers using a multiple-internal-reflection configuration to study thin-film a-Si anodes in typical LIBs conditions, something that was not possible with the previous configurations (Figure 18). ${ }^{152}$ The finding provided real-time and quantitative information on the NIBAM surface and bulk phenomena. Later, Koo et al. used this same configuration to investigate the lithiation mechanism of methylated a-Si anodes (i. e. a-Si $i_{1-x}\left(\mathrm{CH}_{3}\right)_{\mathrm{x}}: \mathrm{H}$ with several methyl contents). ${ }^{153}$ Although the conductivity reduction of this NIBAM limited the range investigated, they observed that a better electrochemical performance was obtained for higher methyl content.

Other in situ FTIR configuration, which was named diffuse reflectance infrared Fourier transform spectroscopy (DRIFTS), was used by Yohannes et al. to investigate the effect of fluoroethylene carbonate additive on the SEI formation over Si-based NIBAM into LIBs. ${ }^{154}$ DRIFTS provided information about the SEI and the reductive decomposition species formed during several cycles.

A novel technique, related with FTIR and complementary, is the called IR-visible Sum Frequency Generation (SFG) vibrational spectroscopy. It uses two laser beams that spatially and temporally overlap at a surface of a material or the interface between two media. This modality is especially useful to infer the composition, orientation distributions, and structural information of molecules at different interfaces. In LIBs, this technique has been recently reported by Horowitz et al. to optimize the electrolyte composition. For this, they tested several fluorine-based additives in order to produce stable formation of SEI on silicon-based NIBAM during successive chargedischarge cycles. ${ }^{155,}{ }^{156}$ In addition, Olson and co-workers have applied this interface-sensitive operando spectroelectrochemical SFG characterization technique to identify electrolyte redox products in LIBs using an anode based on active Si-NPs. ${ }^{157}$ They concluded that this advance electroanalytical method provides precise insight on the stability of high-capacity anodes.

To the date and to the best of our knowledge, related bibliography about the use of in situ FTIR for the investigation of different types of NIBAMs over SIBs and KIBs has not been reported yet. And despite of all efforts made to date, in situ FTIR alone needs to be supported with other operando imaging techniques to further understand phenomenon that occurring.

\subsubsection{Optical Microscopy}

Optical microscopy is not sufficient to monitor microstructural changes because the resolution of optical microscopy is fundamentally restricted by the diffraction limit of visible light. But it can investigate electrochemically induced macroscopic structural changes of electrodes and dendritic lithium formation. Direct observation of the particles of NIBAMs as they react in situ is necessary to build an understanding of the growth characteristics in large-volume-change active materials. Although researchers have devised suitable analytical techniques to study this lithiation/delithiation process through in situ methods, such as in situ $\mathrm{AFM},{ }^{158}$ in situ $\mathrm{TEM},{ }^{78}$ and so on. However, these analytical techniques are very expensive, and may not resolve the whole picture of NIBAM volume expansion.

By introducing the capacity of optical microscopy above, considerable interest has been devoted to understanding volume change and dendritic lithium formation during electrochemical lithiation of NIBAMs by using in situ optical microscopy. Duay and co-workers have utilized optical microscope equipped with a Bertrand lens to monitor of the diffraction efficiency and the volume expansion/contraction process of amorphous silicon (a-Si) thin-film anodes during the lithiation/delithiation process based on photolithographic methods. ${ }^{159}$ They found when the diffraction efficiency along with optical constants obtained from in situ spectroscopic ellipsometry is utilized, volume changes of the active materials can be deduced. The method allows for real-time monitoring of the volume change at charge/discharge cycles. They demonstrated the technique can provide needed insight into understanding the lithium alloying reaction and subsequent induced capacity fade during the cycling of alloying anodes in lithium-ion batteries.

More recently, Feng et al. utilized the operando color microscopy to investigate the lithiation process in pure (a-Si:H) and methylated (a$\left.\mathrm{Si}_{1-\mathrm{x}}\left(\mathrm{CH}_{3}\right)_{\mathrm{x}}: \mathrm{H}\right)$ amorphous silicon thin layers. They found the first lithiation of a-Si $i_{1-x}\left(\mathrm{CH}_{3}\right)_{x}: \mathrm{H}$ is spatially non-uniform. The lithiation starts at a limited number of locations then expands radially, forming circular lithiation spots because of the high resistivity of methylated silicon and the existence of low-resistance point defects. ${ }^{160}$

The optical microscopy can also measure other NIBAMs. The isotropic expansion as a function of lithium content of an amorphous particle can be measured with confidence through in situ observations of a single particle of active material in plain view, which agrees with the value as measured by in situ AFM. ${ }^{161}$ Using in-situ optical microscopy, it was revealed that self-healing of cracks in Si-Al electrodes occurred in two steps by arresting of micro-cracks at the $\mathrm{Si} / \mathrm{Al}$ interfaces and closure of the cracks at the end of the electrochemical cycle. ${ }^{162}$ Furthermore, the optical microscopy can record the evolution of the lithium plating between the electrodes positioned in co-planar configuration (Figure 19). ${ }^{163}$ 

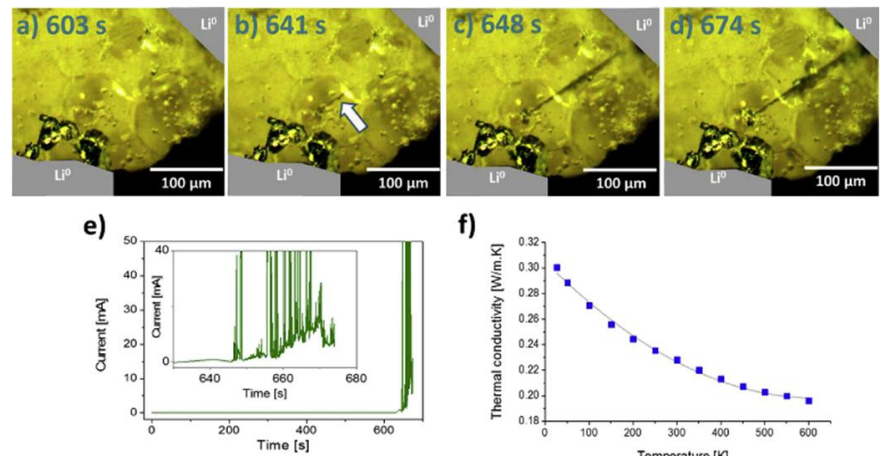

f)

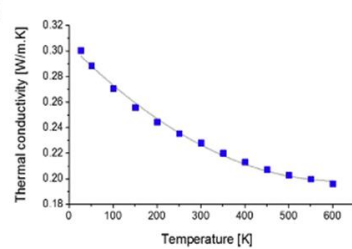

Figure 19. In-operando characterization of the garnet surface after $5 \mathrm{~V}$ bias polarization in co-planar symmetric configuration. In-situ optical microscopy of a Ga-doped LLZO ceramic pellet with Li foil contacts (colored in grey for guidance), for which snapshots were taken at 603s (a), 641s (b), 648s (c) and $674 \mathrm{~s}$ (d) (The complete video can be found in SI). The crack begins at $641 \mathrm{~s}$ (white arrow) and short-circuits the two Li metal electrodes at $648 \mathrm{~s} \mathrm{e}$ ) Current evolution during the polarization experiment. f) Thermal conductivity data for a Ga-doped LLZO ceramic pellet from 25 to $600{ }^{\circ} \mathrm{C}$. Reproduced with permission $^{163}$. Copyright 2019, Elsevier.

\subsubsection{Multi-beam Optical Stress Sensor (MOSS)}

In MOSS a laser beam is reflected off the substrate surface; when the substrate acquires a curvature due to film stress, the position of the reflected beam on a photo-detector changes. By measuring the translation of the beam position, the substrate curvature is calculated, which can be converted into stress thickness values based on Stoney's equation. This technique provides direct correlations among the potential, current and stress, and is capable of measuring the reversible (generated by alloying de-alloying or intercalation de-intercalation processes) and irreversible stresses (generated by irreversible SEI, inorganic residues and dead lithium formation) generated during electrochemical processes.

Sethuraman and co-workers researched real-time measurements of stress evolution in a silicon thin-film electrode during electrochemical lithiation and delithiation through the MOS technique to demonstrate that such stress measurements can be used to calculate mechanical dissipation in the electrode during lithiation/delithiation, and compare it to polarization losses. ${ }^{164}$ They reported in situ measurements of stress evolution in a silicon thin-film electrode during electrochemical lithiation and delithiation by using the Multi-beam Optical Sensor (MOS) technique. Upon lithiation, due to substrate constraint, the silicon electrode initially undergoes elastic deformation, resulting in rapid rise of compressive stress. The electrode begins to deform plastically at a compressive stress of ca. $-1.75 \mathrm{GPa}$; subsequent lithiation results in continued plastic strain, dissipating mechanical energy. Upon delithiation, the electrode first undergoes elastic straining in the opposite direction, leading to a tensile stress of ca. 1 $\mathrm{GPa}$; subsequently, it deforms plastically during the rest of delithiation. The plastic flow stress evolves continuously with lithium concentration. Thus, mechanical energy is dissipated in plastic deformation during both lithiation and delithiation, and it can be calculated from the stress measurements; they showed that it is comparable to the polarization loss. Upon current interrupt, both the film stress and the electrode potential relax with similar timeconstants, suggesting that stress contributes significantly to the chemical potential of lithiated-silicon. Then they measured the change in the biaxial modulus of $\mathrm{Si}$ anodes as a function of $\mathrm{Li}$ concentration with in situ MOSS analysis. ${ }^{165}$ They found the biaxial modulus drops substantially from ca. $70 \mathrm{GPa}$ for $\mathrm{Li}_{0.32} \mathrm{Si}$ to ca. $35 \mathrm{GPa}$ for $\mathrm{Li}_{3.0} \mathrm{Si}$. A simple rule of mixtures was seen to agree well with the measurements. Hence, they reasoned that other relevant mechanical properties (e.g., ductility and fracture toughness) would also change substantially, which has implications for realistic modelling of the mechanics of $\mathrm{Si}$ anodes to predict their cycle life.

Recently, Chen et al. presented an in situ system to study the evolution of both morphology and stress in the silicon thin film electrode during lithiation and delithiation by MOSS (Figure 20). ${ }^{166}$ They designed special in situ cell with two observation windows, thus both the curvature and color of the silicon thin-film electrodes upon lithiation and delithiation processes can be measured by MOSS (Figure 20E). Combining in situ characterization, they obtained the accurate stress of the $\mathrm{Li}_{\mathrm{x}} \mathrm{Si}$ film (Figure 20F). The tendency of the stress evolution is similar to the curvature of cell voltage. The thickness of the $\mathrm{Lix}_{\mathrm{x}} \mathrm{Si}$ film in the traditional theory was overestimated result from the neglect of the side reaction in the in situ batteries. Thus, the stress calculated from the modified thickness is higher than that calculated from the theoretical expansion factor, and the max compressive stress is 1.53 GPa which is increased $19.5 \%$ than that calculated from the traditional model. Furthermore, the max tensile stress is also increased $24.0 \% \%$ to $0.88 \mathrm{GPa}$ during delitiation process.
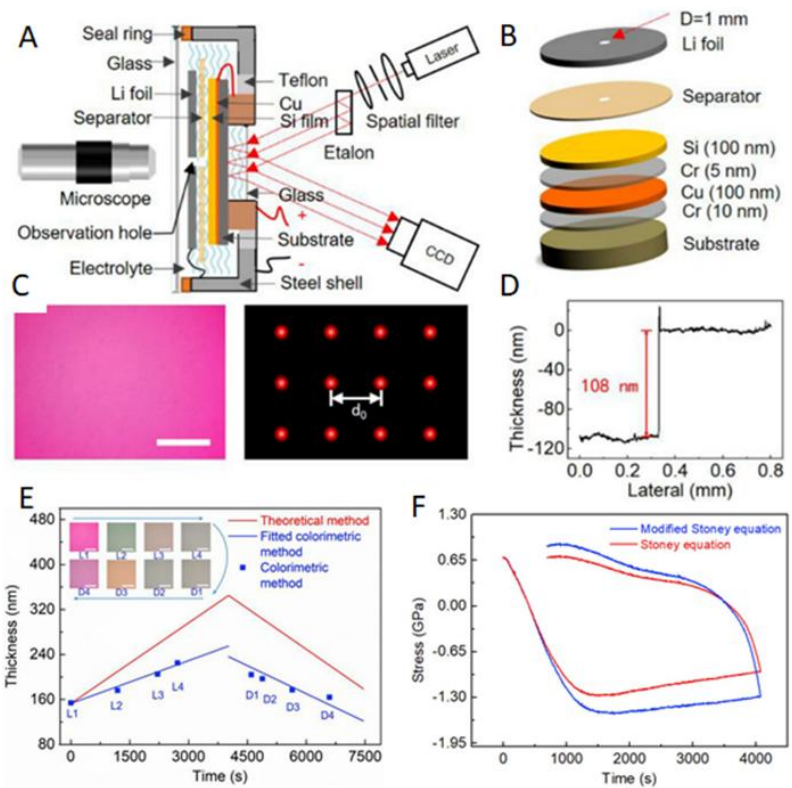

Figure 20. (A) In situ stress measurement/optical microscopy system; (B) Schematic of the layered structure of the silicon film, the observation hole diameter is $1 \mathrm{~mm}$; (C) Optical microscope records color evolution and MOS setup monitors the relative change in the laser-spot spacing; (D) The silicon film thickness is about $108 \mathrm{~nm}$. The scale bar is $50 \mu \mathrm{m}$. (E) Thickness evolution of LixSi calculated from theory (red line) and measured by colorimetric method (blue dots), and the key points color evolution. The scale bar is $30 \mu \mathrm{m}$. (F) Stress evolution of LixSi calculated from Stoney equation (red line) and modified Stoney equation (blue line). Reproduced with permission $^{166}$. Copyright 2019, Elsevier.

MOSS can be also applied to study the stress in various alloys as well as in intercalation-based anodes, such as tin, ${ }^{167}$ aluminium, ${ }^{168}$ germanium, ${ }^{169,}{ }^{170}$ and $\mathrm{Li}_{4} \mathrm{Ti}_{5} \mathrm{O}_{12} .{ }^{171}$ 


\subsubsection{Sum Frequency Generation-Vibrational Spectroscopy (SFG-VS)}

SFG-VS is the coherent second-order nonlinear optical process where a signal at the frequency of the sum of a visible photon and an infrared (IR) photon is generated from the simultaneous interaction of the two photons with a nonlinear optical medium. SFG-VS is intrinsically surface/interface selective and essentially background free, which can be used to probe chemically specific information of the molecular surface or interface, and obtain quantitative information about the molecular (group) number density, molecular (group) orientation and conformation molecular vibrational and electronic spectroscopy, and kinetics and dynamics of chemical changes and relaxations, etc. of molecular surfaces and interfaces.

For LIBs, in situ SFG-VS has previously been used to study SEI formation. ${ }^{172}$ The cyclability of silicon anodes in LIBs is affected by the reduction of the electrolyte on the anode surface to produce SEI. One of the key steps for a major improvement of LIBs is unravelling the SEI's structure-related diffusion properties as charge and discharge rates of LIBs are diffusion-limited. Horowitz et al. ${ }^{173}$ utilized the method to probe the molecular composition of the SEI surface species under various applied potentials. They found that even at open circuit potential (OCP) DEC decomposes on the silicon (100)hydrogen surfaces to form Si-ethoxy bonds. Later, they employed the technique combining with XRR to explore the first monolayer and to probe the first several layers of electrolyte, respectively. The results suggested that the addition of FEC to EC solution causes the first monolayer to rearrange itself more perpendicular to the anode surface, while subsequent layers are less affected and tend to maintain their surface-parallel arrangements (Figure 21). ${ }^{174}$

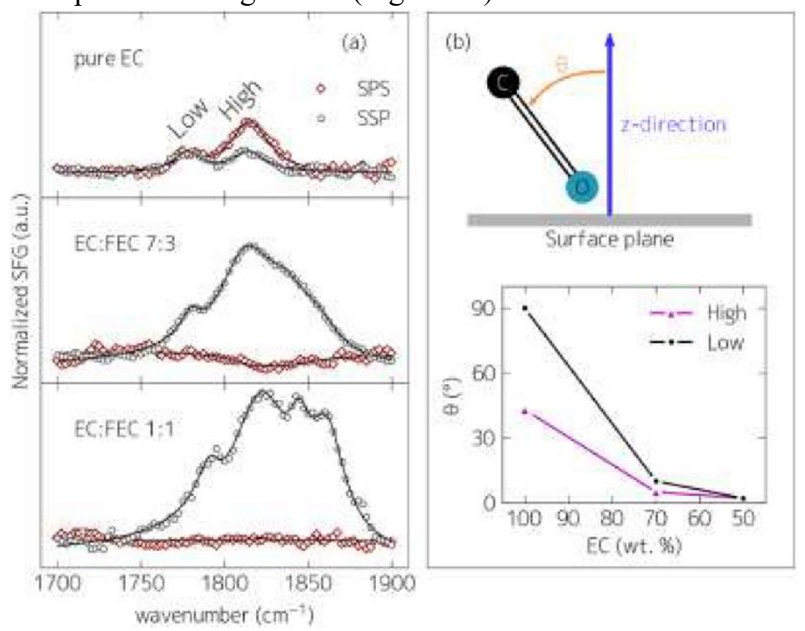

Figure 21. (a) SFG spectra of pure EC and EC/FEC mixtures at open circuit potential probed at SSP (open circles), sensitive to perpendicular aligned adsorbates and SPS (open diamonds), sensitive to parallel aligned adsorbates. For clarification, we assign low and high to EC carbonyl group vibrations. The addition of FEC produces two more peaks at $\sim 1840 \mathrm{~cm}^{-1}$ and $\sim 1855 \mathrm{~cm}^{-1}$. (b) Top, an illustration showing the $\mathrm{C}=\mathrm{O}$ vector and its angle $(\theta)$ from the normal to the surface $(Z)$. Note that $\theta=0$ when the $C=O$ is perpendicular to the $\mathrm{X}-\mathrm{Y}$ surface. Bottom, the $\mathrm{C}=\mathrm{O}$ bond angle with respect to the surface normal as a function of FEC wt \% content. Reproduced with permission ${ }^{174}$. Copyright 2018, American Chemical Society.
These techniques are non-invasive arising from their advantages of high sample penetration, sensitivity to low atomic number elements and low neutron energy. Furthermore, being a zero charge particle, neutron interact with nuclei, while X-ray and gamma ray interact with electrons of an atom, which makes neutron techniques specific to isotopes, especially lighter ones. ${ }^{175}$ Therefore, some neutron techniques are essential for NIBAM research, such as small angle neutron scattering, neutron diffraction, neutron reflectometry, neutron radiography/tomography, neutron depth profiling.

\subsubsection{Small angle neutron scattering (SANS)}

SANS provides the changes of the nanostructure (such as size, volume, and shape of particles) via using high penetration depth. ${ }^{176}$ Thus, the combination of SANS and electrochemical testing is a noninvasive method for monitoring morphology changes of NIBAM during electrochemical cycling, including the volumetric expansion and the generation of fractures.

He and co-authors applied in situ SANS combining with SEM and XRD to evaluate the electrochemical swelling and recovery of the individual nanoscale grains for $\mathrm{Cu}_{3} \mathrm{P}$ and $\mathrm{Sn}_{4} \mathrm{P}_{3} .{ }^{177}$ The scattering model reads as Equation (1)

$$
\begin{aligned}
\frac{\mathrm{d} \sum}{\mathrm{d} \Omega}(Q)= & A_{\text {Porod }} Q^{-4}+A_{\text {globule }} \\
& \times\left(\exp \left(-\frac{1}{3} Q^{2} R_{\mathrm{g}}^{2}\right)+\frac{8 \cdot \operatorname{erf}^{12}\left(1.06 Q R_{\mathrm{g}} / \sqrt{6}\right)}{Q^{4} R_{\mathrm{g}}^{4}}\right)
\end{aligned}
$$

Since the latter two effects are well below the incoherent background level, they focused the interpretation on the two amplitudes $\mathrm{A}_{\text {Porod }}$ and Aglobule. As shown in Figure 22A, B, during the lithiation of $\mathrm{Cu}_{3} \mathrm{P}$, no structure changes and new phase can be seen before $1.2 \mathrm{~V}$. When the cell is lithiated further to $0.01 \mathrm{~V}$, the Aporod value decreases, which indicates an overall surface area reduction of the active material particles. This suggests a uniform coating of the SEI on the surface of the active material particles which may also partly fill the void space between each small grain. They found that a smoother surface is formed possibly because a mixture of compounds of SEI and the products from active material at fully lithiated state (including $\mathrm{Li}_{\mathrm{x}} \mathrm{P}$ and $\mathrm{Cu}^{0}$ ) are formed on the particles surface in the individual wellseparated small grains. $\mathrm{Li}^{+}$ions are fully extracted from the $\mathrm{Li}_{x} \mathrm{Cu}_{y} \mathrm{P}$ intermediate phase, resulting in $\mathrm{Cu}_{3} \mathrm{P}$ at the end of delithiation process. However, in contrast with the $\mathrm{Cu}_{3} \mathrm{P}$ electrode, $\mathrm{Li}^{+}$ions can further react with $\mathrm{Sn}$ to form an intermetallic phase (such as $\mathrm{Li}_{4.4} \mathrm{Sn}$ ), resulting in more than $420 \%$ volume expansion (Figure 22C, black rectangles). The mechanical failure of $\mathrm{Sn}_{4} \mathrm{P}_{3}$ material during the lithiation process is expected to arise as a result of such large volume expansion. No small particles form on the surface after the lithiation process, thus further demonstrating that the particles for both $\mathrm{Li}_{x} \mathrm{Sn}$ and $\mathrm{Li}_{3} \mathrm{P}$ were largely expanded (Figure 22D, black rectangles). During delithiation, $\mathrm{Li}^{+}$ions are extracted from the swollen structures and this process creates additional detached domains that contribute to a considerable larger surface (Figure 22C, red circles) and also explain the formation of small grains (Figure 22D, red circles).

\subsection{Neutron techniques}



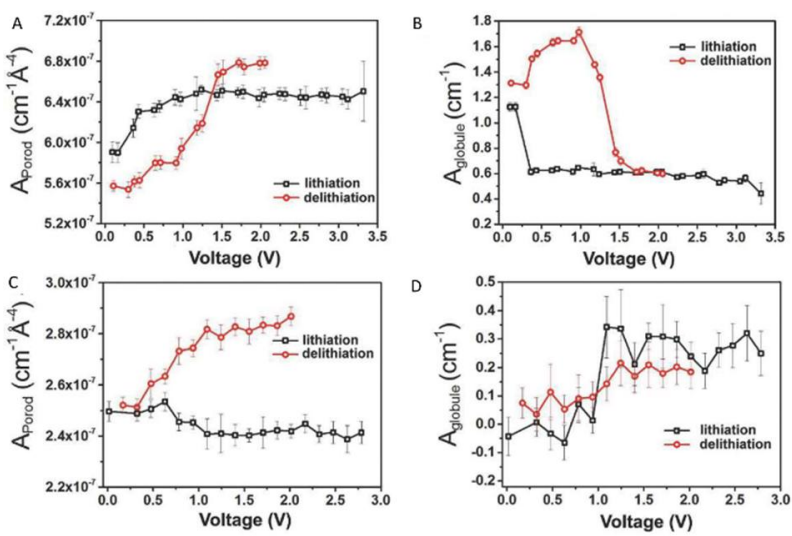

Figure 22. The fitting parameters of the SANS curves of $\mathrm{Cu}_{3} \mathrm{P}$ electrode: $(\mathrm{A})$ $\mathrm{A}_{\text {Porord }}$ and (B) $\mathrm{A}_{\text {globule }}$ as a function in the voltage range of $0.01-2 \mathrm{~V}$ at a sweep rate of $0.2 \mathrm{mV} \mathrm{s}^{-1}$. The essential fitting parameters of the SANS curves of $\mathrm{Sn}_{4} \mathrm{P}_{3}$ electrode: (C) $\mathrm{A}_{\text {Porord }}$ and (D) $\mathrm{A}_{\text {globule }}$ as a function in the voltage range of 0.01$2 \mathrm{~V}$ at a sweep rate of $0.2 \mathrm{mV} \mathrm{s}^{-1}$. Reproduced with permission. Copyright 2017, American Chemical Society.Although in situ SANS is useful techniques to analyse new insights of the nanostructural changes of NIBAMs reacting with lithium via conversion and alloying, we can't nearly find more examples.

\subsubsection{Neutron diffraction}

In situ neutron diffraction has been used to determine the structure changes, phases transitions, and the formation of SEI layers during battery cycling. Comparing to in situ XRD technology, in situ neutron diffraction can obtain unique information in detecting the structural evolution of electrode materials due to neutrons are sensitive to the light elements and can easily distinguish elements with similar atomic number. Another important advantage of neutron diffraction over $\mathrm{XRD}$ is the higher penetration depth of neutrons in comparison to Xrays, making neutron diffraction truly bulk sensitive and suitable for large cell formats, whereas XRD is only suitable for thin samples. Furthermore, magnetic properties can be investigated.

Colin et al. first developed the in situ electrochemical cell for neutron diffraction of electrode materials. They selected $\mathrm{Li}_{4} \mathrm{Ti}_{5} \mathrm{O}_{12}$ as model material and followed the evolution of the structural parameters of $\mathrm{Li}_{4} \mathrm{Ti}_{5} \mathrm{O}_{12}$ during the entire cycle. A small decrease of the cell parameter from $8.3636 \AA$ to $8.3580 \AA$ accompanied by an increase of the Ti-O bond length was observed. The $\mathrm{Li}$ occupation during its migration from the $8 \mathrm{a}$ to the $16 \mathrm{c}$ site in $\mathrm{Li}_{7} \mathrm{Ti}_{5} \mathrm{O}_{12}$ by examining the evolution of the $\mathrm{Li}_{7} \mathrm{Ti}_{5} \mathrm{O}_{12} 773$ and 666 reflection intensities with battery charge. ${ }^{178}$ Additionally, Pang and co-workers ${ }^{179}$ elucidated the simultaneous structural phase evolution of both the cathode and anode by the time-resolved in-situ neutron diffraction in the $\mathrm{LiNi}_{0.5} \mathrm{Mn}_{1.5} \mathrm{O}_{4}|| \mathrm{Li}_{4} \mathrm{Ti}_{5} \mathrm{O}_{12}$ full cell. They found the Ti oxidation state corralled to the intensity in the $\mathrm{O}$ positional parameter during charge/discharge cycling. Then they demonstrated an increase in the rate of $\mathrm{Li}$ incorporation into the anode rather than a change in the migration pathway as a result of the particle size reduction of $\mathrm{Li}_{4} \mathrm{Ti}_{5} \mathrm{O}_{12}$ anode. ${ }^{180}$ The lattice of different anodes during continuous lithiation undergoes expansion followed by a gradual contraction and then expansion again. $\mathrm{Li}$ at the $8 \mathrm{a}$ site is replenished faster than the rate of transfer of Li to the $16 \mathrm{c}$ site. Fourier-difference nuclear density maps of both anodes suggest that $32 \mathrm{e}$ sites are involved in the diffusion pathway of Li. They also found that while the initial expansion of the lattice upon lithiation is not reflected in the trend of $\mathrm{TiO}_{6}$ octahedral distortion, the gradual lattice-contraction experienced during further lithiation is strongly correlated to the trend of the $\mathrm{TiO}_{6}$ distortion, and associated with the repopulation of $\mathrm{Li}$ at the $16 \mathrm{c}$ site in the anode. ${ }^{181}$

Du et al. ${ }^{182}$ demonstrated that anatase $\mathrm{TiO}_{2}$ is electrochemically active when they explored a composite anatase $\mathrm{TiO}_{2} / \mathrm{Li}_{4} \mathrm{Ti}_{5} \mathrm{O}_{12}$ electrode by in situ neutron diffraction and found that $\mathrm{Li}$ can be selectively intercalated at $1.7 \mathrm{~V}$ to form $\mathrm{Li}_{\mathrm{x}} \mathrm{TiO}_{2}$. $\mathrm{Li}$ insertion/extraction in anatase $\mathrm{TiO}_{2} / \mathrm{Li}_{\mathrm{x}} \mathrm{TiO}_{2}$ occurs on the order of 15 min with applied currents of $2 \mathrm{~mA}$. The process is a two-phase reaction, with increasing quantities of lithiated $\mathrm{TiO}_{2}$ forming during discharge.

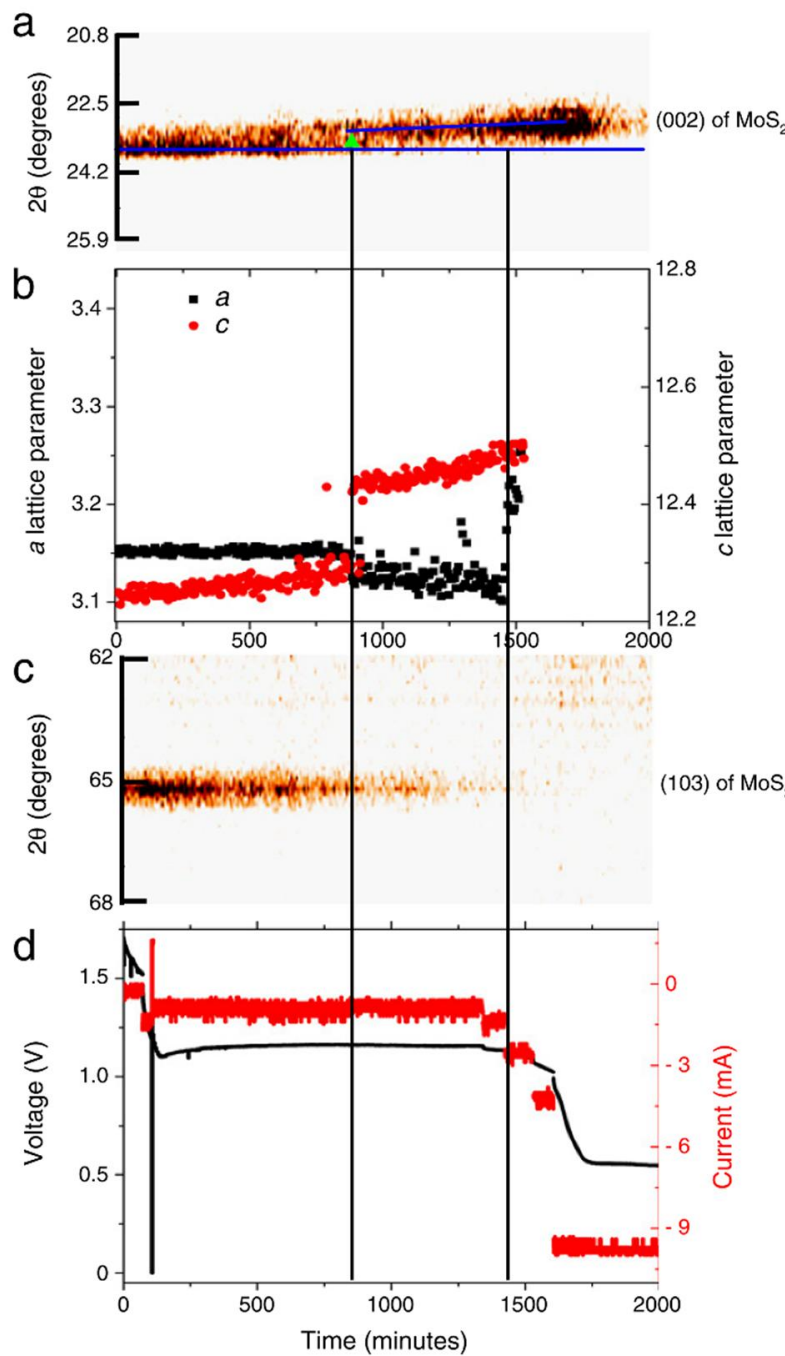

Figure 23. Selected regions $\left(20.8^{\circ} \leq 2 \theta \leq 25.9^{\circ}\right.$ and $\left.62^{\circ} \leq 2 \theta \leq 68^{\circ}\right)$ of the in-situ ND data obtained from the $\mathrm{MoS}_{2}$ cell as a function of time showing discharge evolution of: (a) The (002) peak with a reference line (guide to the eye) showing the $2 \theta$ shift. (b) Rietveld-refined a (black squares) and c (red circles) lattice parameters of $\mathrm{MoS}_{2}$. (c) The (103) $\mathrm{MoS}_{2}$ peak. (d) Measured voltage (black line) and applied current (red) correlated to the ND data. The reflections intensities are scaled. Reproduced with permission ${ }^{183}$. Copyright 2011, Elsevier.

Furthermore, in situ neutron diffraction can be applied to study other NIBAMs. Sharma and co-workers ${ }^{183}$ optimised the electrochemical 
cells for in situ neutron diffraction study of the $\mathrm{MoS}_{2}$ anode electrode during discharge(Figure 23). They found Li-insertion into $\mathrm{MoS}_{2}$ via disappearance of the (103) reflection and increase in the d-spacing of the (002) reflection (Figure 23a). The changes in the diffraction patterns begin at the $1.1 \mathrm{~V}$ plateau and are complete during the $0.5 \mathrm{~V}$ plateau (Figure 23c). Sequential Rietveld-refinement indicates the presence of an intermediate lithiated phase $\left(\mathrm{Li}_{x} \mathrm{MoS}_{2}\right)$ between $\mathrm{MoS}_{2}$ and $\mathrm{LiMoS}_{2}$. They observed the disappearance of all reflections for the $\mathrm{MoS}_{2}$, corresponding to the loss of long-range order, during the $0.5 \mathrm{~V}$ plateau and no new diffraction peaks appear with further electrochemical cycling. Their results showed a transformation from long-range ordered $\mathrm{MoS}_{2}$ to short-range ordered $\mathrm{LiMoS}_{2}$, which were confirmed using ex-situ synchrotron X-ray and neutron diffraction.

\subsubsection{Neutron reflectometry (NR)}

NR is a scattering technique that can be used conveniently to in-situ monitor thin films, interfaces and surfaces, especially explore SEI formation process and composition of NIBAM electrodes. ${ }^{184}$ In NR, the specular reflection of neutrons from the surface or interface is measured as a function of the wave vector transfer perpendicular to the sample surface. It allows quantitative determination of the SEI properties as a function of electrolyte composition during charging/discharging processes and provides direct insight of the intercalation kinetics and intercalation pathways with high spatial and temporal resolution. Additionally, NR requires very smooth surfaces/interfaces.

Jerliu and co-workers investigated of the intercalation of lithium into amorphous silicon electrodes in lithium-ion batteries by in situ NR. ${ }^{185-}$ 188 They found that the penetration of lithium into amorphous silicon can effectively be monitored during charging and discharging by changes in the scattering length density. The results indicate a constant $\mathrm{Li}$ concentration inside the electrode. The measurements allow us to detect the volume expansion/reduction of amorphous silicon during lithiation/de-lithiation caused by the modification of the silicon host by lithium. Additionally, during de-lithiation the formation of a several nanometer thin lithium rich surface layer can be observed, which might be identified with the SEI. Finally, irreversible charge losses after complete de-intercalation were found in the experiments, which are caused by the existence of the lithium rich surface layer and small amounts of residual lithium in the silicon electrode.

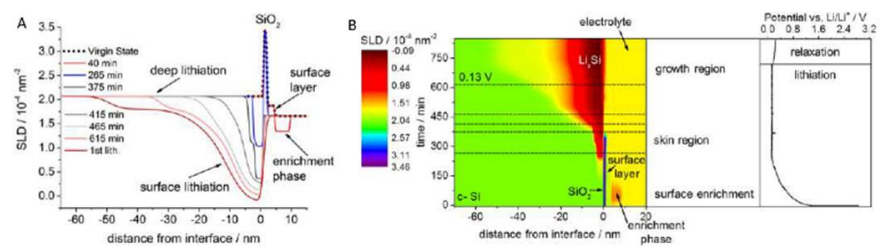

Figure 24. Characteristic SLD profiles of the virgin state (black dots), taken during lithiation and at the end of first lithiation (dark red) corresponding to the fits to the NR data. After $\sim 370 \mathrm{~min}$, the native $\mathrm{SiO}_{2}$ vanishes and the SLD further decreases with time inside the silicon electrode (dark gray). SLD values between $\sim 1.80 \times 10^{-4}$ and $-0.13 \times 10^{-4} \mathrm{~nm}^{-2}$ are reached, equivalent to the deep and surface lithiation, respectively. (B) SLD profiles of the first lithiation as a function of time and distance from the interface. The right-hand side of the diagram displays the potential of the working electrode vs $\mathrm{Li} / \mathrm{Li}^{+}$. At the beginning of lithiation, four regions are visible: c-Si (green), the native $\mathrm{SiO}_{2}$ layer (violet), a surface layer (light green), and the electrolyte (yellow). The following processes can be divided into three phases: surface enrichment (orange), surface lithiation (red/dark-red), and deep lithiation (red-yellow). Black dotted lines depict the example SLD curves shown in Figure (A) at a

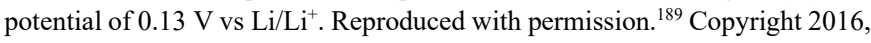
American Chemical Society.

Seidlhofer et al. studied on the electrochemical incorporation of lithium into crystalline silicon by an operando NR from the $\langle 100\rangle$ surface of a silicon single crystal used in an electrochemical cell. ${ }^{190}$ The strong scattering contrast between $\mathrm{Si}$ and $\mathrm{Li}$ due to the negative scattering length of $\mathrm{Li}$ leads to a precise depth profile of $\mathrm{Li}$ within the $\mathrm{Si}$ anode as a function of time (Figure 24). The operando cell can be used to study the uptake and the release of Li over several cycles. Lithiation starts with the formation of a lithium enrichment zone during the first charge step (Figure 24). The total depth of penetration was less than $100 \mathrm{~nm}$ in all experiments. The thickness of the highly lithiated zone is the same for the first and second cycle, whereas the thickness of the less lithiated zone is larger for the second lithiation (Figure 25). A surface layer of lithium $(\mathrm{x} \sim 1.1)$ remains in the silicon electrode after delithiation (Figure $25 \mathrm{~b}$ ). The uptake of $\mathrm{Li}$ can be divided into a highly lithiated zone at the surface (skin region) $(\mathrm{x} \sim 2.5$ in $\mathrm{Li}_{\mathrm{x}} \mathrm{Si}$ ) and a much less lithiated zone deep into the crystal (growth region) ( $\mathrm{x} \sim 0.1$ in $\mathrm{Li}_{\mathrm{x}} \mathrm{Si}$ ) (Figure $25 \mathrm{~b}$ ). Moreover, a SEI is formed and dissolved during the entire cycling. They demonstrated that neutron reflectivity allows the tracking of the kinetics of lithiation and delithiation of silicon with high spatial and temporal resolution.

(a)

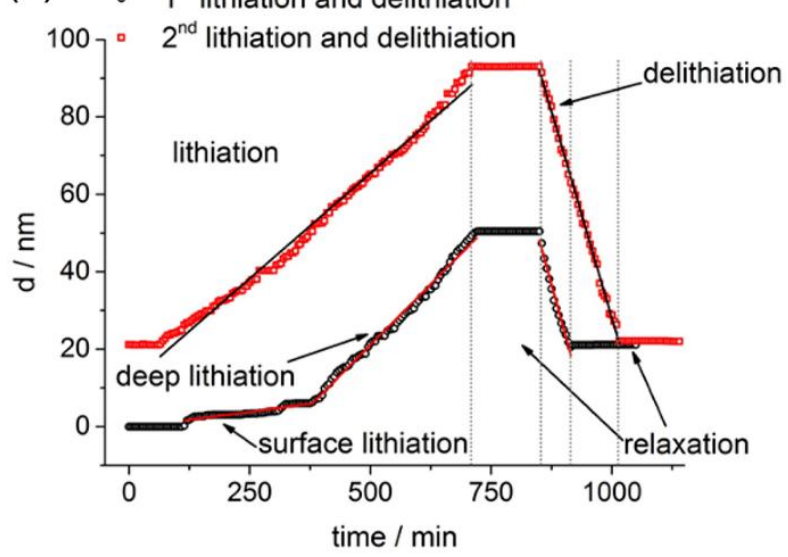

(b) $\longrightarrow-1$ st lithiation and delithiation

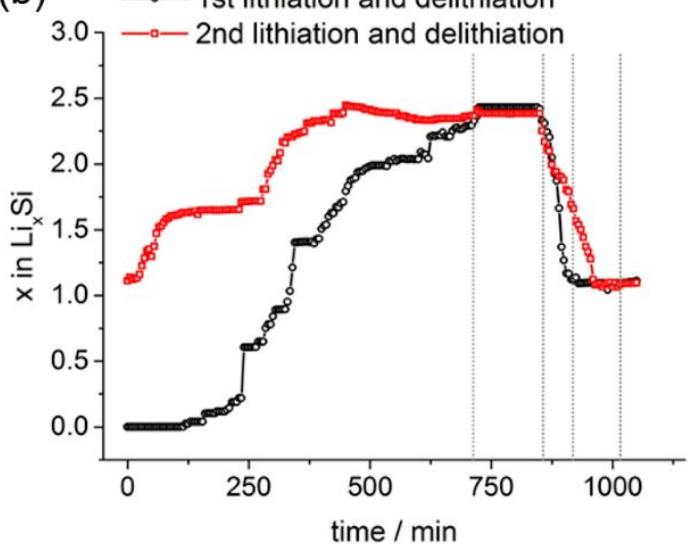

Figure 25. (A) Changes of the complete $\mathrm{Li}_{x} \mathrm{Si}$ layer thickness $\mathrm{d}_{\mathrm{s}+\mathrm{g}}$ with time for the first and second lithiation and delithiation. The surface and deep lithiation are displayed by different slope kinetics of electrode penetration by lithium. 
(B) Changes of the lithium content $\mathrm{x}$ in $\mathrm{Li}_{\mathrm{x}} \mathrm{Si}$ in the skin region of the first and second lithiation. A theoretical error of $5 \%$ was calculated from the average of 10 SLD values. Reproduced with permission ${ }^{189}$. Copyright 2016, American Chemical Society.

\subsubsection{Neutron Radiography/Tomography}

Neutron radiography technique is a valuable tool, which has been applied to image liquid in gas flow channels and ion transport in porous materials. In neutron radiography, a detector is placed behind an illuminated object to measure the change in neutron transmission through that object because the neutron interactions with the battery reduce the number of transmitted neutrons. The neutron cross-sections of various materials represent their attenuation which are different due to neutron scattering and absorption. In 2D neutron radiography images, the denser parts appear as white and the lighter parts appear darker on a photographic plate due to the different levels of scattering and absorption of radiation. The $3 \mathrm{D}$ reconstruction of the radiographic images is known as tomography. The methods can be used to investigate macroscopic changes of batteries, such as volume changes, ion diffusion and distribution, and so on.

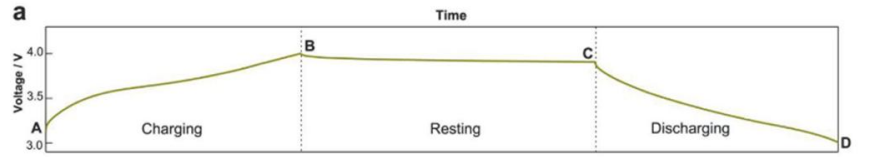

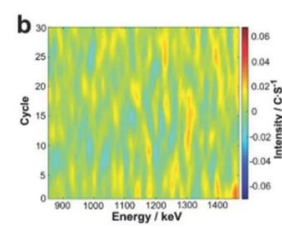

e

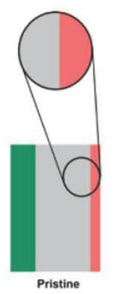

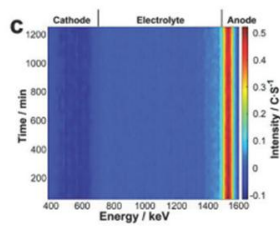
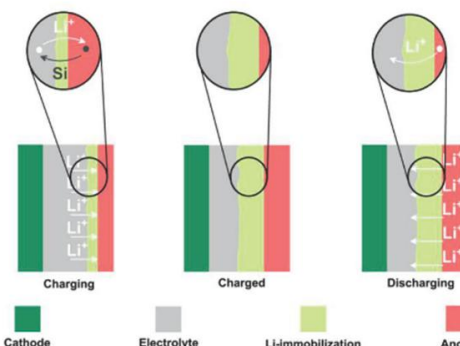

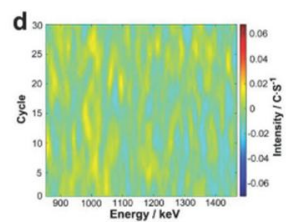

Figure 26. Snapshots from an in operando neutron radiography series taken during the first discharge: The sequence of letters from A to F refers to elapsing time (see also the time label). The region encircled in yellow is the original area that covers the initial electrode; from a to $\mathrm{h}$, different states of the $\mathrm{Si}$ particle during first lithiation as marked in the $3^{\text {rd }}$ red circular region in $\mathrm{A}, \mathrm{G}$, attenuation changes of regions 1-3 defined in $\mathrm{A}$ as s function of slice number (discharge time). Note that the values in $\mathrm{G}$ are the relative change of the neutron attenuation coefficient. The scale bar in $\mathrm{A}$ is $400 \mu \mathrm{m}$, the scale bar in a $200 \mu \mathrm{m}$ long. Reproduced with permission ${ }^{191}$. Copyright 2017, American Chemical Society.

It is obvious that in operando and nondestructive diagnostic techniques with the ability to temporally and spatially visualize ion de/insertion processes inside batteries may open up new opportunities for high-capacity and high-power electrode materials for nextgeneration energy storage systems. By complementary in operando Xray radiography and neutron radiography measurements, Sun et al. investigated and visualized the initial lithiation in silicon-electrode lithium-ion batteries. ${ }^{191}$ The in operando neutron radiography confirmed the results of X-ray radiography, which is significant volume expansion of Si particles and the Si electrode during the first discharge, and many $\mathrm{Si}$ particles never undergo electrochemical reactions (Figure 26). It firstly showed the process of Li alloying with the Si electrode during initial lithiation. Furthermore, these results demonstrated that complementary X-ray and neutron radiography is a powerful tool to investigate the lithiation mechanisms inside Sielectrode based lithium-ion batteries.

\subsubsection{Neutron Depth Profiling (NDP)}

NDP is a low-energy neutron-based technique and can easily detect the real-time information of ion density as a function of electrode depth during battery cycling. It initiates the nuclear reaction of the constituent elements in the analyzed surface or interface through generating $4 \mathrm{He}$ as alpha and $3 \mathrm{H}$ as triton particles with well-defined energies of $2055 \mathrm{keV}$ and $2727 \mathrm{keV}$, respectively. ${ }^{192}$ These generated particles lose their energy through the electrode layers before surfacing to the detector. The distribution of the atoms can be explored by detecting their loss of energy. Therefore, it can help in getting a better signal strength during NDP through using an isotopic ionlabelled electrode material and electrolytes.
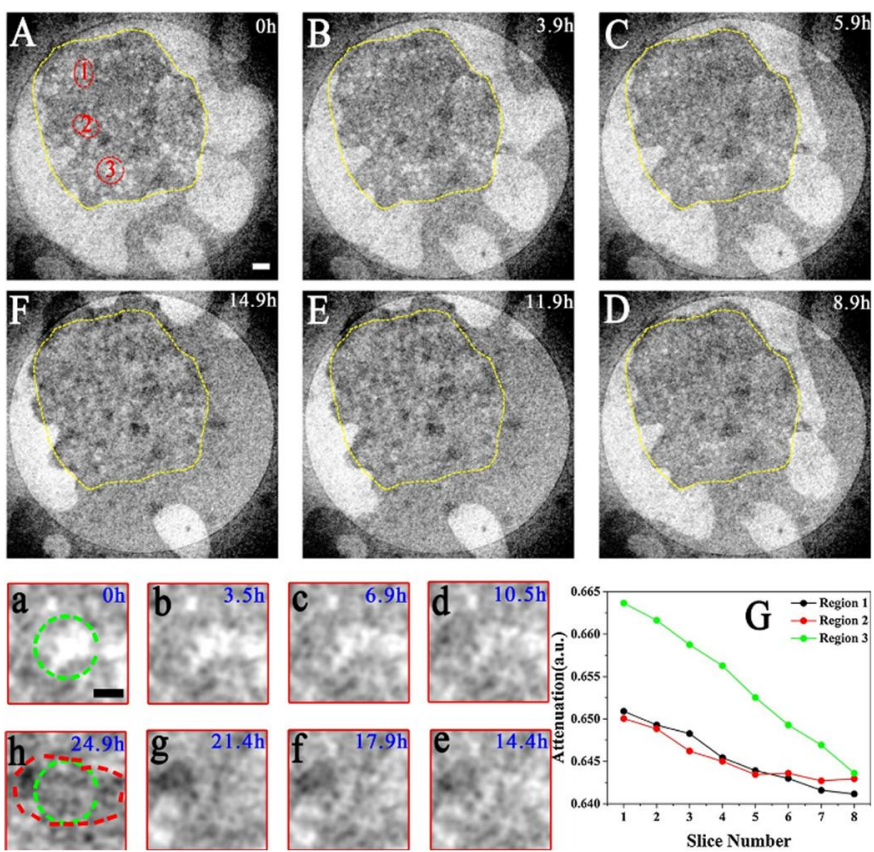

Figure 27. a) Voltage charging, resting, and discharging curve of a $\mathrm{Si}-\mathrm{Li}_{3} \mathrm{PO}_{4}$ $\mathrm{LiCoO}_{2}$ solid-state, thin film battery during which the NDP spectra has been continuously collected. c) In operando spatial energy profiles during the 1260 min of resting after the first charging process. The increment spatial images for b) fully charged and d) fully discharged batteries during the first 30 cycles, of which the images were obtained by subtracting the spectra for each cycle before the charging (B-A) and discharging (D-C) processes have started, respectively. e) Schematic representation of the evolution of the degradation mechanism of a $\mathrm{Si}-\mathrm{Li}_{3} \mathrm{PO}_{4}-\mathrm{LiCoO}_{2}$ thin film battery upon cycling, involving the formation of the Li-immobilization interlayer, originating from the $\mathrm{Si} / \mathrm{Li}_{3} \mathrm{PO}_{4}$ interface. Reproduced with permission. ${ }^{193}$ Copyright 2018 , WILEY.

By utilizing in situ NDP, Liu et al. probed the onset of lithiation in a high-capacity Sn anode and visualized the enrichment of Li atoms on the surface followed by their propagation into the bulk. ${ }^{194}$ The delithiation process shows the removal of Li near the surface, which leads to a decreased coulombic efficiency, likely because of trapped $\mathrm{Li}$ within the intermetallic material. The developed in situ NDP provides exceptional sensitivity in the temporal and spatial 
measurement of $\mathrm{Li}$ transport within the battery material. This diagnostic tool opens up possibilities to understand rates of $\mathrm{Li}$ transport and their distribution to guide materials development for efficient storage mechanisms. ${ }^{195}$ Additionally, Liu et al. confirmed the preferential lithiation of Sn nanoparticles as anode material when they demonstrated the feasibility of $\mathrm{Al}$ as anode current collector in LIBs by in situ NDP. ${ }^{196}$

For SSBs, like all rechargeable battery systems, they inevitably suffer from capacity losses during operation. Chen et al. used in operando NDP method to investigate the degradation mechanism of SSBs, thin film $\mathrm{Si}-\mathrm{Li}_{3} \mathrm{PO}_{4}-\mathrm{LiCoO}_{2}$ batteries. ${ }^{193}$ As shown in figure 27, they found that the capacity losses in these thin film batteries are mainly related to lithium immobilization in the solid-state electrolyte, starting to grow at the anode/electrolyte interface during initial charging. The Li-immobilization layer in the electrolyte is induced by silicon penetration from the anode into the solid-state electrolyte and continues to grow at a lower rate during subsequent cycling. Therefore, the amount of free moveable lithium ions is reduced, leading to the pronounced storage capacity decay.
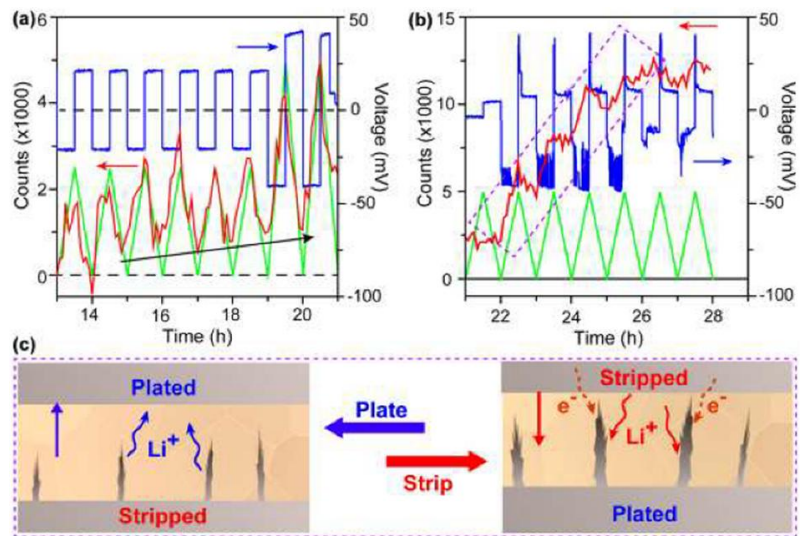

Figure 28. In situ NDP measurement for diagnosing short-circuit in a $\mathrm{Li} /$ garnet/Li symmetric cell during $\mathrm{Li}$ plating and stripping. The zoom-in images of the voltage pro fi le (blue), charge curve (green), and the integrated NDP count curve (red) of symmetric cell cycling at (a) the predicted and (b) the "dynamic short-circuit" stages, respectively. (c) Schematic to illustrate the "dynamic short-circuit" mechanism during Li plating and stripping. Reproduced with permission. ${ }^{197}$ Copyright 2017, American Chemical Society.

The garnet-based solid state electrolyte (SSE) is considered a promising candidate to realize all solid state lithium (Li) metal batteries. However, critical issues require additional investigation before practical applications become possible, among which high interfacial impedance and low interfacial stability remain the most challenging. Therefore, Wang et al. used in situ NDP to monitor the $\mathrm{Li}$ distribution and transport of garnet-based solid state cells during cycling. ${ }^{197}$ They observed several interesting $\mathrm{Li}$ morphologies including the 3D porous lithiated CNT, Li chunks, and mushroomlike Li structures in the Li plated Li/garnet/CNT asymmetric cell due to the poor point contact between a conventional Li metal host and solid state electrolyte. To explain this phenomenon, a reversible layer is distinguished near the CNT/garnet interface where Li can be plated and stripped reversibly. When Li plates beyond the reversible layer, it becomes "dead" Li and starts to accumulate, which agrees well with NDP measurements and corresponding voltage profiles of asymmetric cells. For $\mathrm{Li} /$ garnet/Li symmetric cells, both the voltage profiles and
NDP measurements show better reversibility with higher maximum current densities because of the conformal contact between $\mathrm{Li}$ and garnet SSE. The diagnostic capability of in situ NDP measurement was demonstrated in a short-circuit prediction (Figure 28). A unilateral "dynamic short-circuit" mechanism is proposed according to the increasing NDP count in symmetric cells prior to apparent short-circuiting in the voltage profile. Therefore, ionically conductive materials are necessary for the implementation of a solid state battery. A 3D mixed electron-ion conductive framework is preferred as a $\mathrm{Li}$ metal host to increase the contact area, shorten the Li diffusion distance, and overcome the anticipated volume change.

\subsection{Magnetic techniques}

Magnetic techniques can be used to probe the chemical environment in electrodes in situ. ${ }^{198}$ The most commonly researched battery system contains either lithium $\left({ }^{7} \mathrm{Li}\right)$ or sodium $\left({ }^{23} \mathrm{Na}\right)$ chemical species, which are paramagnetic in nature and responsive to an applied magnetic field. Nowadays, the commonly used magnetic techniques are nuclear magnetic resonance spectroscopy, magnetic resonance imaging, Mössbauer spectroscopy, electron paramagnetic resonance spectroscopy and electron magnetic measurements. ${ }^{199,200}$

\subsubsection{Nuclear Magnetic Resonance (NMR) Spectroscopy}

NMR occurs when the nuclei of an atom aligned to an applied field are induced to change their spin orientation with respect to the applied field due to the absorption of energy in the form of a radio frequency wave. NMR is helpful in monitoring the structural changes over an electrode, electrolyte and their interfaces. ${ }^{175}$ The applied magnetic field causes the precession of spinning nuclei, which generates an oscillating electric field with a definite frequency. If a similar radio frequency is supplied, then it gets absorbed by the processing nuclei, resulting in changes of the spin. This condition is called resonance, and spinning nuclei are said to be in resonance with the applied electromagnetic waves. The energy absorbed during this transition is a function of the nucleus type and its chemical environment in the molecule. In situ NMR studies of batteries are performed on the entire battery, by using a coin cell design, a flat sealed plastic bag, or a cylindrical cell. The battery is placed inside the NMR coil, leads are connected to a potentiostat, and the NMR spectra are recorded as a function of state of charge. The in situ NMR technique can be traced back to fifty years ago. ${ }^{201}$ However, the application of in situ NMR in lithium-ion batteries started long back after the commercialization of the lithium-ion battery in 1991 .

For LIBs, in situ NMR was initially used to research the intercalation of carbon-based materials, such as graphite, corannulene, porous carbon, and so on. ${ }^{202-204}$ Then, Key and co-workers monitored/identified the changes in the short-range order that occur during the first discharge of crystalline silicon in an actual working LIB by in situ ${ }^{7} \mathrm{Li}$ and ${ }^{29} \mathrm{Si}$ NMR spectroscopy combining with MAS and ex situ NMR spectroscopy. ${ }^{205}$ They demonstrated that in situ ${ }^{7} \mathrm{Li}$ NMR can be used to capture changes that cannot be readily obtained through ex situ methods. The first discharge occurs via the formation of isolated $\mathrm{Si}$ atoms and smaller $\mathrm{Si}-\mathrm{Si}$ clusters embedded in a $\mathrm{Li}$ matrix; the latter are broken apart at the end of the discharge, forming isolated Si atoms. They observed that a spontaneous reaction of the lithium silicide with the electrolyte, which results in self-discharge and potential capacity loss. The rate of this self-discharge process is 
much slower when CMC (carboxymethylcellulose) is used as the binder.

Ogata et al. developed a strategy for performing in situ, in operando ${ }^{7} \mathrm{Li}$ NMR spectroscopy, which can be used to study in unprecedented detail the kinetics of the electrochemical lithiation and delithiation reactions that occur in technologically relevant $\mathrm{nm}$-sized Si based anodes under realistic cycling conditions (with careful voltage/current controls) over multiple cycles. ${ }^{206}$ Combining with systematic electrochemical characterization, ex situ XRD, ex situ NMR and DFT, they found that the phases formed on delithiation strongly depend on the rate of lithiation and allow us to rationalize the large voltage hysteresis seen in these systems. Such understanding is of high relevance to practical cycling strategies. Very recently, Freytag et al. utilized ${ }^{7} \mathrm{Li}$ NMR to study both $\mathrm{Si}$ and a-SiO using jelly-roll-type battery, showing the intrinsic difference between the lithiation of those two materials. ${ }^{207}$ They also found no crystalline $\mathrm{Li}_{15} \mathrm{Si}_{4}$ phase at the end of lithiation, which contrasts with the lithiation of $\mathrm{Si}$, where the $\mathrm{Li}_{15} \mathrm{Si}_{4}$ phase disrupts the structure in the high lithiation regime, leading to long-term capacity loss.

Bhattacharyya et al. first monitored metallic lithium deposited on the lithium metal anode by using in situ NMR in combining with electrochemical cycling and were able to resolve distinct signals from bulk $\mathrm{Li}$ metal in the electrodes and from the $\mathrm{Li}$ metal microstructures. ${ }^{208}$ Using simple calculations based on the skin depth of metallic structures under radiofrequency excitations, they found that it is possible to quantify the amount of mossy/dendritic lithium formed. Later, Chang et al. found the deposition of microstructure of one $\mathrm{Li}$ isotope onto the bulk metal strip of the other isotope, which can definitively assign the signals of the microstructure and explore the skin depth effect. ${ }^{209}$

Furthermore, Wang et al. first used in situ ${ }^{7} \mathrm{Li}$ NMR spectroscopy to investigate lithium sulfur electrochemistry, which is capable of quantitatively capturing the evolution of the soluble and precipitated lithium(poly)sulfides during electrochemical cycling. Through deconvolution and quantification, every lithium bearing species was closely tracked and a four-step soluble lithium polysulfide mediated lithium sulfur electrochemistry was demonstrated in a never seen before detail. Significant irreversible accumulation of $\mathrm{Li}_{2} \mathrm{~S}$ is observed on the Li metal anode after 4 cycles due to sulfur shuttling.

For SIBs, Bayley et al. ${ }^{210}$ first used in situ ${ }^{23} \mathrm{Na}$ NMR to quantitatively investigate $\mathrm{Na}$ metal deposits with a morphology associated with an extremely high surface area, the deposits continually accumulating, even in the case of galvanostatic cycling (Figure 29). Two regimes for the electrochemical cycling of $\mathrm{Na}$ metal are apparent that have implications for the use of $\mathrm{Na}$ anodes: at low currents, the $\mathrm{Na}$ deposits are partially removed on reversing the current, while at high currents, there is essentially no removal of the deposits in the initial stages. At longer times, high currents show a significantly greater accumulation of deposits during cycling, again indicating a much lower efficiency of removal of these structures when the current is reversed. Stratford et al. first used operando ssNMR revealed the possibility of further sodium storage within the $\mathrm{Na}_{15} \mathrm{Sn}_{4}$ structure, formed toward the end of discharge, as an off-stoichiometry phase $\mathrm{Na}_{15+\times} \mathrm{Sn}_{4}$, in a process analogous to that observed in the lithium-silicon system. ${ }^{211}$

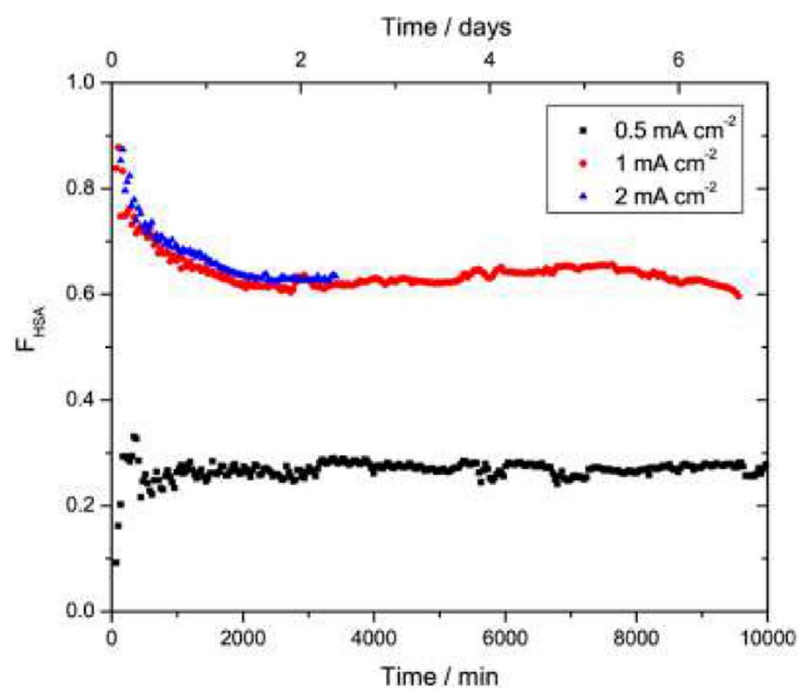

Figure 29. Fraction of high surface area $\left(\mathrm{F}_{\mathrm{HSA}}\right) \mathrm{Na}$ during galvanostatic cycling at various current densities. A ratio of 1 indicates completely rough deposition while 0 indicates smooth deposition. Reproduced with permission. ${ }^{210}$ Copyright 2016, American Chemical Society.

\subsubsection{Magnetic Resonance Imaging (MRI)}

MRI is a natural extension of NMR and provides spatial information about tagged NMR-active nuclei. MRI generates a 3D matrix image of a cell on the time scale of the electrochemical reaction using specific chemical shift points, which provide sufficient temporal resolution to analyze the ongoing electrochemical process. Therefore, it can measure the spatial distribution of a particular concentration of species that are NMR active.

The first application of the in situ MRI technique for the real-time observation of lithium-ion batteries was performed by Nakayama et al. in 2010 using a solid-state electrode Swagelok-type cell assembly. ${ }^{212}$ In 2012 Chandrashekar et al. demonstrated that ${ }^{7} \mathrm{Li}$ MRI method enabled a completely non-invasive visualization and characterization of the changes that occur on battery electrodes and in the electrolyte. ${ }^{213}$ They used ${ }^{7} \mathrm{Li}$ MRI to investigate the locations of the Li-metal deposits. They revealed that the dendritic Li features have a narrow range of chemical shifts near $270 \mathrm{ppm}$, while mossy microstructures have broader peaks covering a large frequency range from 262 to $274 \mathrm{ppm}$. Then, Chang and co-workers found a direct correlation to be made between the growth of dendrites with changes in the electrolyte concentration gradient by ${ }^{7} \mathrm{Li} \mathrm{MRI}$. An analysis is performed to investigate the validity of Chazaviel's model and the accuracy of the theoretical Sand's time and to understand the impact of Sand's time on the dendrite growth in this system. They demonstrated that accurate estimates of the start time of dendrite growth can be obtained using chemical shift images of the ${ }^{7} \mathrm{Li}$ metal signal, which agree with theoretical Sand's time predictions at high current densities. At low currents, dendrites begin to grow more quickly than predicted, suggesting that other mechanisms are dominant in cells charged at low current densities.

Although the method described above is helpful for direct monitoring of metallic microstructure growth, it requires specific NMR-active nuclei of a particular metal for in situ/operando MRI. Ilot et al. ${ }^{214}$ developed a method to overcome the hindrance by the disclosure of an indirect approach to observe the effects of dendrites formed on its 
encompassing electrolyte, rather than monitoring morphological changes of the Li nucleus itself. They monitored the changes in the electrolyte intensity (Figure 30A) and their correlation with volume changes (Figure 30B) in the electrolyte. These electrolyte volume changes can be correlated with the volume of fractal-like growth of metal (lithium) microstructures (Figure 30C) assuming all the deposited lithium takes a dendritic or mossy shape. The volume of the deposited lithium can be estimated from Faraday's law and matched with the NMR data. They revealed that the growth of dendrites is not unidirectional, and after the dendrite growth, the morphology of the already formed structure does not change further throughout the experiment. Furthermore, the indirect MRI approach described above provides a "one nucleus fits all" solution, and can be extended to other electrode material, such as $\mathrm{Zn}, \mathrm{Na}$, and $\mathrm{Mg}$. Effects of advanced electrode designs, electrolytes, and separators on dendrite formation and growth can be assessed in future battery designs using this approach conveniently.
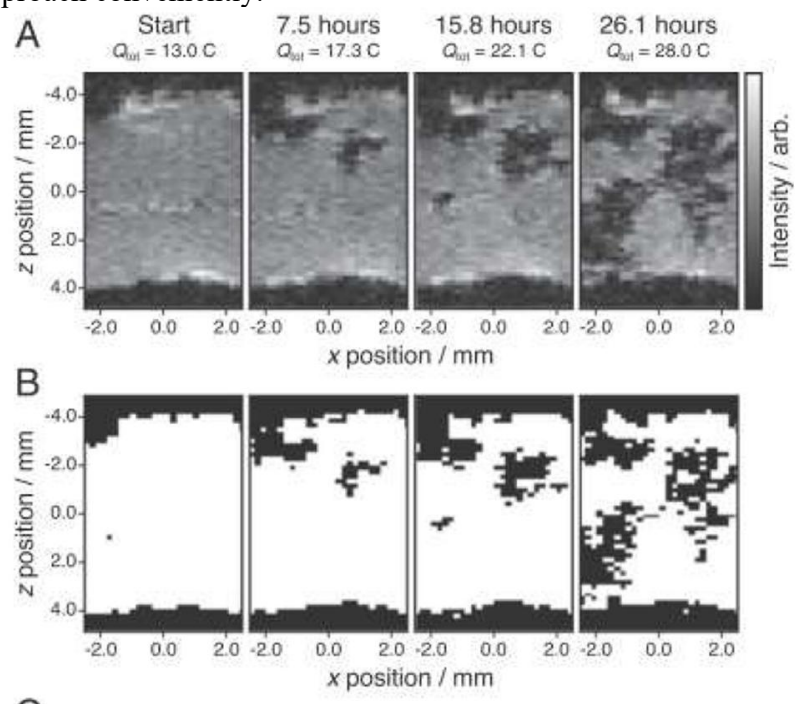

C

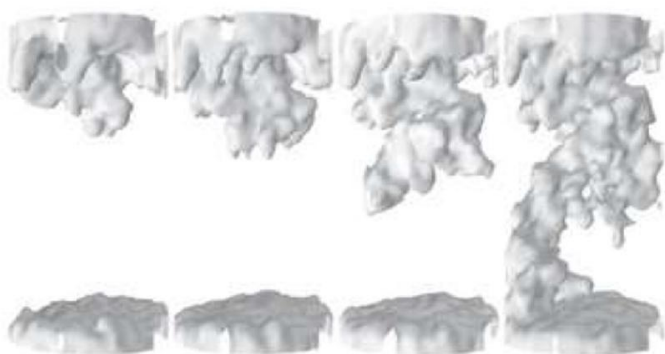

Figure 30. In situ ${ }^{1} \mathrm{H}$ 3D FLASH imaging results from the electrochemical cell, with each 3D image acquired in $16 \min 40 \mathrm{~s}$. The cell was charged at $50 \mu \mathrm{A}$ $\left(0.16 \mathrm{~mA} \mathrm{~cm}^{-2}\right)$ for $72 \mathrm{~h}$ before these measurements and then charged at 160 $\mu \mathrm{A}\left(0.51 \mathrm{~mA} \mathrm{~cm}^{-2}\right)$ for the times specified, with the total charge applied also given in coulombs. (A) Two-dimensional slices from four time points. (B) Segmented images of the results from A, where I threshold $=0.2 \mathrm{I}$ max. (C) Three-dimensional segmented images of the same time points with an additional Gaussian filter applied to smoothen the visualization. Reproduced with permission. ${ }^{214}$ Copyright 2016, Proceedings of the National Academy of Sciences.

\subsubsection{Mössbauer spectroscopy}

Mössbauer spectroscopy is highly sensitive nuclear technique for investigating the local electronic structure. It works on the resonance emission and the absorption of gamma rays from the nucleus of an atom without the loss of energy from nucleus recoil. Thus, Mössbauer spectroscopy can provide information about the locally chemical environment of a nucleus, including valence state changes, the effect of different ligands on a particular atom, and the magnetic environment of the material. Furthermore, Mössbauer spectroscopy can probe the relative concentration of different spin state of metal elements, which cannot be detected by other electron structure techniques, such as XPS and XAS.

As representative examples for LIBs, Sougrati et al. used operando ${ }^{117} \mathrm{Sn}$ Mössbauer spectroscopy and XRD to measure the structural change of the TiSnSb during charging/discharging process. ${ }^{215}$ They found the TiSnSb undergoes a conversion process, leading simultaneously to the formation of $\mathrm{Li}-\mathrm{Sb}$ and $\mathrm{Li}-\mathrm{Sn}$ alloys during the first discharge. At the end of the discharge, $\mathrm{Li}_{3} \mathrm{Sb}$ and $\mathrm{Li}_{7} \mathrm{Sn}_{2}$ were identified. Once the first discharge is achieved, both phases were shown to form $\mathrm{Ti}-\mathrm{Sn}$ or $\mathrm{Ti}-\mathrm{Sb}$ or $\mathrm{Ti}-\mathrm{Sn}-\mathrm{Sb}$ nanocomposites. The good electrochemical performance of TiSnSb (compared to $\mathrm{Sn}$ and $\mathrm{Sb})$ seems to be a consequence of the presence of the non-active metal. They demonstrated that the structural feature of the pristine material clearly impacts both the mechanism involved during the cycling and the corresponding performance.

Some samples for Mössbauer spectroscopy analysis are very expensive to prepare, thus synchrotron Mössbauer pectroscopy (SMS) is necessary because it can offer much higher signal-to-noise ratio and allows for characterization under operando conditions. Fehse et al. first used operando SMS to investigate battery material, $\mathrm{FeSb}_{2}$. Coupled with operando Sb K-edge XAS, they found that sodiation leads not only to the formation of amorphous $\mathrm{Na}_{3} \mathrm{Sb}$ but also to the formation of $\mathrm{Fe}$ nanoparticles which contain a fraction of $\mathrm{Sb}(\leqslant 10 \%)$ dissolved in their lattice. Upon desodiation, the Fe does not bind back to $\mathrm{Sb}$ but remains in the form of metallic nanoparticles while $\mathrm{Sb}$ forms amorphous/nanoconfined $\mathrm{Sb}$ particles, hence $\mathrm{FeSb}_{2}$ is never recovered. Thus, after the first discharge, the observed mechanism should be considered as an alloy-type rather than as a conversion reaction. Even though the iron nanoparticles are electrochemically inactive, the formation of nano-dispersed metallic iron particles promotes cycling stability and enhances cycling performance thanks to their ability to mitigate volume changes and prevent $\mathrm{Sb}$ agglomeration. ${ }^{71}$

\subsubsection{Electron paramagnetic resonance (EPR)/ electron spin resonance (ESR)}

EPR or ESR is an analytical technique that shows paramagnetic behaviour of material due to presence of unpaired electron. ESR is more sensitive than NMR because unpaired electron spin is excited rather than nuclear spin. It has been applied to estimate electrode surface area, tack the initialization of microstructural growth, and estimate the paramagnetic reaction intermediates. It should be noted that materials used for cell construction should be transparent to microwave radiation and inactive for EPR. ${ }^{216}$

As representative examples for LIBs, in 2015, Wandt and coworkers ${ }^{217}$ developed a suitable electrochemical cell setup for EPR spectroscopy for the semi-quantitative determination of mossy or dendritic lithium in real-time and under realistic conditions (Figure 31). As mentioned above, EPR results is more sensitive compared to NMR experiments, thus early stages of dendrite formation can be monitored by EPR, as it is bound to excite a thinner layer on the 
surface of the electrode. The authors used two kind of electrolytes, one is normal ones and the other has fluoroethylene carbonate (FEC) additive for reducing the formation of $\mathrm{Li}$ deposition. The line shape analysis of operando EPR demonstrated that the cell containing FEC additive reversibly cycled better than the cell without additive, which is confirmed by ex situ SEM images. Therefore, it is clearly shows that operando EPR spectroscopy is a powerful diagnostic technique, and can yield valuable information.

\subsection{Other techniques}

\subsubsection{Acoustic emission (AE)}

The AE method is a sensitive and nondestructive technique for detecting elastic waves generated by the fracture of electrode materials. ${ }^{218,219} \mathrm{AE}$ is a piezoelectric sensor used to indicate vibrations emitting from a sample that undergoes mechanical deformations by various stresses. ${ }^{218}$ It can be recognized as a valuable instrument to investigate mechanical degradation in negative electrodes of Li-ion batteries, which are objected to severe stresses during electrochemical cycling. ${ }^{220}$ What's more, comparing to most of in situ/operando analytical techniques above, such X-ray, electron, neutron, it is lowcost.
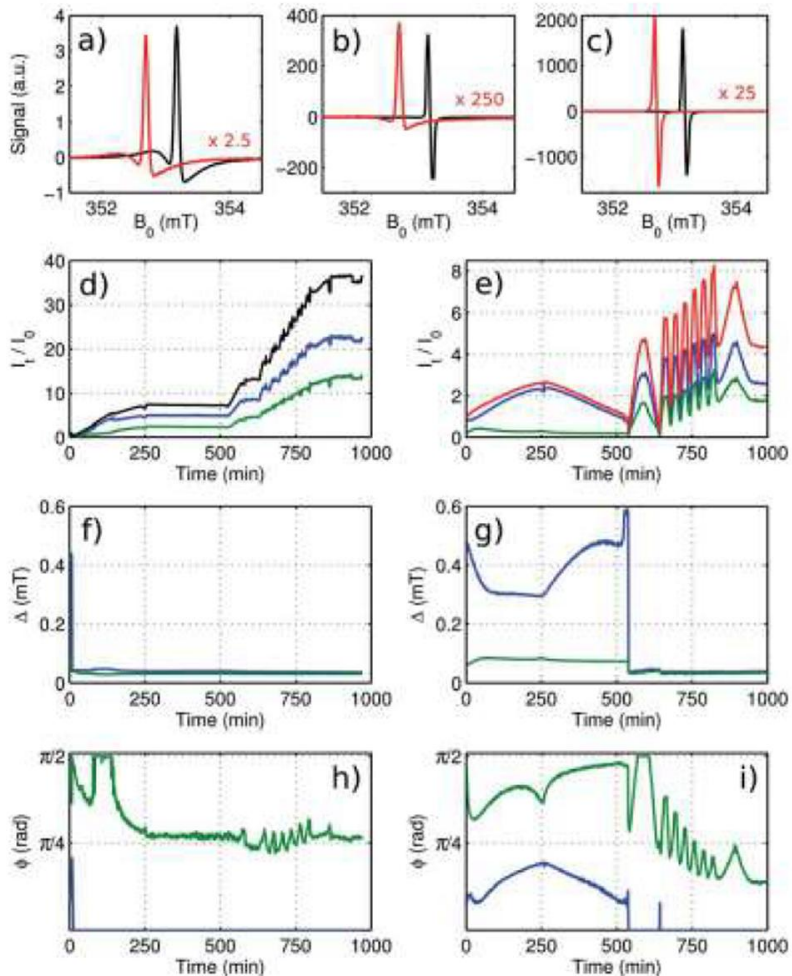

Figure 31. Analysis of time-resolved EPR of metallic Li for cell containing electrolyte without additives (black) and cell containing electrolyte with 10 $w t \%$ FEC (red). The spectra at the beginning of cycling (a), at the end of the first cycle (b), and at the end of the last $3 \mathrm{C}$ cycle (c), are compared. The red spectra are scaled as indicated in each panel. The resonances were fitted using a model with two phase-shifted Lorentzian lines. The amplitudes for the broader (blue) and for the narrower component (green) are shown for the cell without additives (d) and the FEC-containing cell (e). The sum of both components is shown in black and red for each cell. The width and the asymmetry of each component is shown for the cell without additives ( $\mathrm{f}$ and $\mathrm{h}$ ) and for the FEC-containing cell ( $g$ and $\mathrm{i}$ ). Reproduced with permission ${ }^{217}$. Copyright 2015, Royal Society of Chemistry.
AE was first used to monitor particle fracture of positive electrode materials and graphite anodes during charge and discharge. ${ }^{219,}{ }^{221}$ In 2008, AE was utilized to investigate the expansion of $\mathrm{Si}$ based electrodes in Alias' $\mathrm{PhD}$ thesis. ${ }^{222}$ The major source of $\mathrm{AE}$ was identified as the brittle fracture of silicon particles resulting from the alloying reaction that gives rise to $\mathrm{Li}_{\mathrm{x}} \mathrm{Si}$ phases. ${ }^{218}$ The largest number of emissions occurred on the first lithiation, corresponding to surface fracture of the silicon particles, followed by distinct emission bursts on subsequent charge and discharge steps. Furthermore, the AE data well correlated with that calculated following the diffusion equation and equations of elasticity with an appropriate volumetric expansion term. ${ }^{223}$ Recently, the associated mechanical degradation was analyzed via acoustic emission measurements, indicating a higher rate of events during delithiation than lithiation, with most hits detected in the initial cycle (Figure 32). ${ }^{224}$

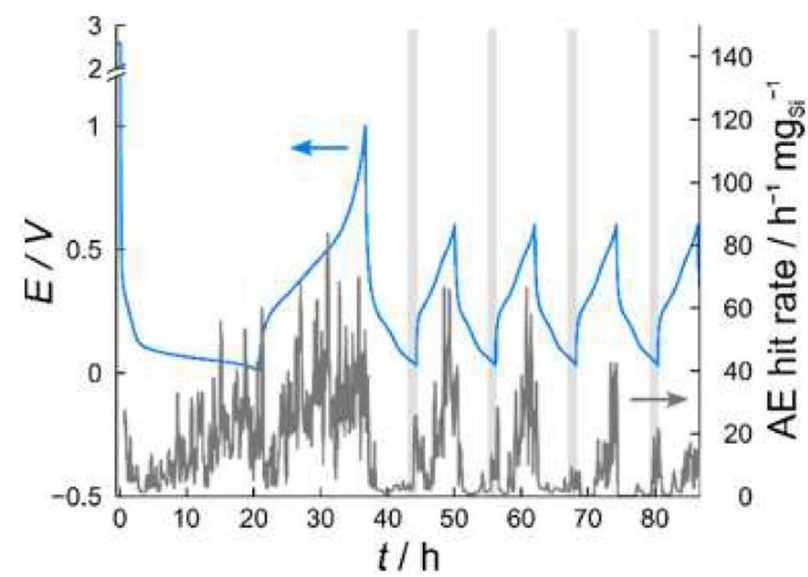

Figure 32. Cell potential (light blue) and $\mathrm{AE}$ hit rate (gray) during $\mathrm{AE}$ measurement on the $\mathrm{Si} / \mathrm{C}$ composite-containing electrode. The cell was assembled with the Li metal and LP57 as the counter electrode and electrolyte, respectively. The region close to the lower cut-off potential is highlighted for clarity. Reproduced with permission ${ }^{224}$. Copyright 2018, American Chemical Society.

\subsubsection{Time-of-flight secondary ion mass spectrometry (ToF- SIMS)}

ToF-SIMS is a highly sensitive surface analytical technique where a pulsed primary ion beam (e.g., Bip) is used to extract secondary ions that are analyzed by time-of-flight spectrometry. Interlaced with a sputtering ion beam (e.g., Csp), compositional depth profiles with excellent depth resolution (monolayer) and high sensitivity (ppb) can be readily obtained. SIMS is able to analyze all the elements in the periodic table, either lighter or heavier. Thus, SIMS is useful in minimizing the cumbersome application of nuclear-based techniques to detect lighter elements, like hydrogen. ToF-SIMS has been applied to cycled electrode materials providing information about the SEI layer and the depth and lateral distribution of species in the bulk electrode materials.

In 2011, Li et al. used ToF-SIMS combing with XPS to characterize the electrode processes induced by lithiation/delithiation of $\mathrm{Sn}-\mathrm{Ni}$ alloy, which is electroplated on a copper substrate, during galvanostatic discharge/charge measurements. ${ }^{225}$ They found that ToF-SIMS depth profiling evidenced an incomplete initial alloying process of lithium ion with $\mathrm{Sn}$ and the resulting partition of the Sn-Ni layer alloy into a fully lithiated outer part and a partially lithiated inner 
layer during the first discharge. After the first cycle, the volume expansion/shrink associated with the alloying/dealloying reaction, also evidenced by ToF-SIMS, irreversibly cracks and divides the Sn$\mathrm{Ni}$ alloy into island-like morphology with gaps filled by the SEI layer. Multicycling (tested up to 9 cycles) amplifies the division of the Sn$\mathrm{Ni}$ alloy layer and the related penetration of the SEI layer as indicated by the increase of trapped lithium and chlorine but with no apparent loss of active material or drop of capacity.
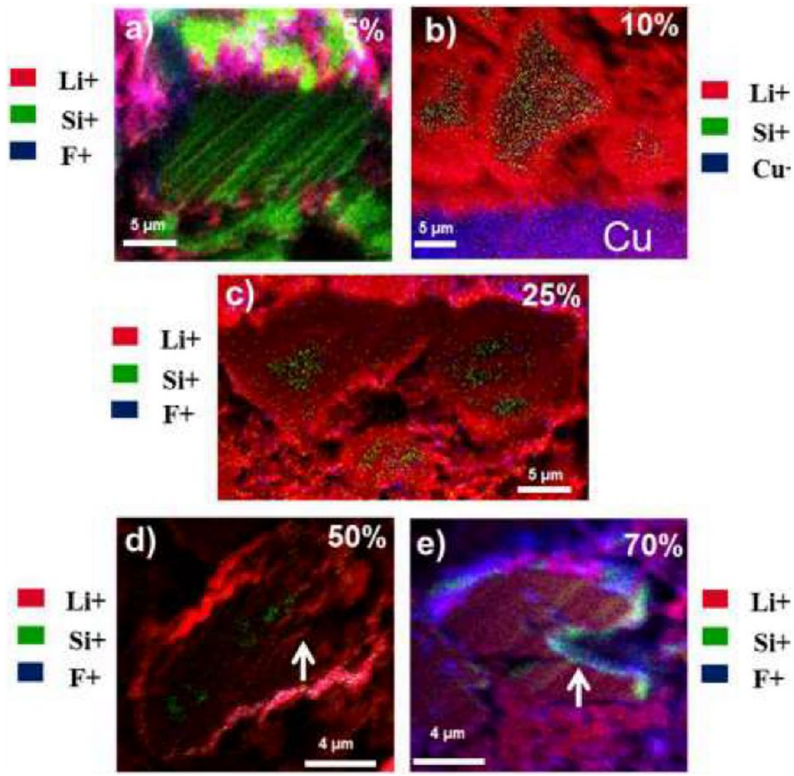

Figure 33. ToF-SIMS chemical mapping of $\mathrm{Li}^{+}$(red), $\mathrm{Si}^{+}$(green) and $\mathrm{F}^{+}$(or $\mathrm{Cu}^{+}$)(blue) of silicon particles cross sections for different states of lithiation: a) surface particle lithiated at $5 \%$ of theoretical capacity; b) particle close to the current collector lithiated at $10 \%$ of theoretical capacity; c) surface particle lithiated at $25 \%$ of theoretical capacity; d) bulk particle lithiated at $50 \%$ of theoretical capacity; e) bulk particle lithiated at $70 \%$ of theoretical capacity Reproduced with permission. ${ }^{227}$ Copyright 2016, American Chemical Society.

For silicon anode, Hüger et al. ${ }^{226}$ utilized SIMS to measure the modification of lithium distribution taking place during galvanostatic lithiation of about $600 \mathrm{~nm}$ thick amorphous silicon film electrodes at low current densities of about $30 \mu \mathrm{A} / \mathrm{cm}^{2}(\sim \mathrm{C} / 14)$, which is confirmed by XPS. They observed a two-step lithiation procedure where the electrode is transformed first into a homogeneously lithiated $\mathrm{Li}_{\mathrm{X}} \mathrm{Si}$ phase with a low Li content of $x \approx 0.3$. During later stages of the lithiation process, the results are in agreement with the penetration of a highly lithiated phase via a moving phase boundary, as also observed for crystalline silicon. Later, a novel approach is developed by using a focused ion beam (FIB) implemented in the analysis chamber of a state-of-the-art ToF-SIMS, where detailed mapping of elements distribution, including lithium inside a silicon particle or in the entire depth of the electrode, can thus be performed to describe precisely the initial lithiation process of silicon particles coupling with Auger electron spectroscopy.227 The samples were transferred directly from a glovebox to the preparation chamber of the TOF-SIMS and then underwent FIB cutting and FIB-TOF-SIMS imaging because lithiated $\mathrm{Si}$ is very sensitive to oxidation. During the first lithiation, the different lithiation states of Si particles were detected (Figure 33). At these early stages of lithiation, lithium does not penetrate in silicon and does not cover the entire silicon surface: all ions have been consumed in parasitic reactions leading to SEI formation (Figure
33A). As far as ToF-SIMS imaging goes, lithiation happens in a single phase (Figure 33E). At $25 \%$ lithiation, the average peak area of $\mathrm{Li}^{+}$ intensity in the TOF-SIMS map was calculated and normalized to that of $\mathrm{Si}^{+}$(Figure 34A), and the evolution of $\mathrm{Lix}_{\mathrm{x}} \mathrm{Si}$ in the shell cross section of a particle was analysed by AES (Figure 34B). The profile contains three different intensity levels. The higher level corresponds to the outer part of the particle and is due to SEI. Then, in the lithiated shell, the intensity remains quite stable before drastically dropping in the core. This profile supports the hypothesis of a constant composition in the lithiated shell, which is separated from the pure silicon core by an interface as sharp as the ToF-SIMS lateral resolution allows to estimate $(\sim 200 \mathrm{~nm})$. In the AES depth profile, there is a lithiation plateau of $\mathrm{Li}_{\mathrm{X}} \mathrm{Si}$ in the shell of a particle, which was in accordance with previous studies. ${ }^{228}$ Based on these profiles and analysis, a first-cycle lithiation model was constructed, providing a reference for quantifying lithiated amorphous Si by employing the TOF-SIMS method (Figure 34C).
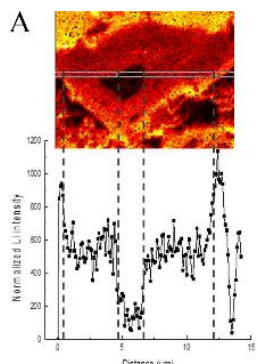

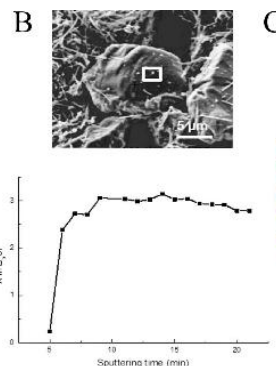

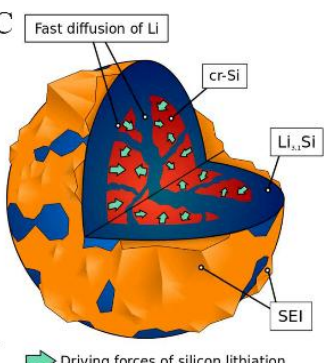

$\rightarrow$ Driving forces of silicon lithiation
Figure 34. (A) ToF-SIMS chemical mapping and intensity of lithium signal normalized to silicon over the width of the cross section for a $25 \%$ lithiated silicon particle. The two horizontal lines on the chemical mapping show the analysis area. (B) Secondary electron imaging and Auger quantification depth profile of the amount of lithium present in the LiSi alloy of the same $25 \%$ lithiated silicon particle. The white rectangle on the secondary electron imaging shows the analysis area. (C) Schematic view of a particle during the first lithiation. Reproduced with permission. ${ }^{227}$ Copyright 2016, American Chemical Society.

\subsubsection{Electrochemical quartz crystal microbalance (EQCM)}

EQCM is a technique used to measure mass changes of model electrodes with a very high accuracy. The investigated electrode is deposited on an oscillating quartz sensor, whose resonance frequency is linearly correlated to the sensor mass according to the well-known Sauerbrey equation in the ideal case of a homogeneous and rigid layer adhered to the sensor surface. The resonance frequency is additionally sensitive to electrolyte viscosity and density changes in vicinity of the sensor. An excellent revision about the development of operando EQCM with dissipation monitoring (EQCM-D) for the systematic characterization of mechanical and morphological properties of electrodes, both for batteries and supercapacitors, has been recently published by Shpigel et al. ${ }^{229}$ Overall, EQCM-D based methodology can be applied for the real-time monitoring of gravimetric, dimensional, and porous structure changes in stiff electrodes and viscoelastic changes in soft electrodes (Figure 35A).

For LIBs, this in situ technique has been initially used to analyse the activation pathway and behaviour of cathodes, ${ }^{230,231}$ and to study the elastic properties of electrolyte additives ${ }^{232}$ and polymeric binders ${ }^{233}$ during lithiation processes. In the latter cases it was successful used 
as a rapid screening method to optimize the electrolyte components for the formation of high-quality SEI. In 2015, Yang et al. employed operando EQCM-D to investigate the SEI formation during charge/discharge cycling onto Sn thin film-based NIBAMs. ${ }^{234}$ From the resulting EQCM-D data (i.e. by the frequency and dissipation results at multiple harmonics) and using the Sauerbrey and Voigt viscoelastic models, they obtained quantitative data about mass, thickness, shear elastic modulus, and shear viscosity. In 2016, Shpigel et al. developed a novel in situ hydrodynamic spectroscopic method for porous electrode structure characterization based on EQCM-D with multiple overtone orders, which was called in situ multiharmonic analysis of EQCM-D. ${ }^{235}$ They validated this novel method using different modified quartz crystal electrodes, such as Au-covered and spray-pyrolyzed porous $\mathrm{LiMn}_{2} \mathrm{O}_{4}$ (LMO) with different geometry and thickness. They finally proposed that this approach could be very useful to investigate both the viscoelastic properties of binder for composite NIBAMs and the intercalation-induced changes for porous electrode structures at a mesoscopic scale. The latter information is not provided by other in situ techniques (i.e. neither by in situ XRD nor operando dilatometry). In 2017, Dargel and co-workers used EQCM-D as gravimetric and viscoelastic probing to perform a realtime study of the SEI formation on a composite $\mathrm{Li}_{4} \mathrm{Ti}_{5} \mathrm{O}_{12}$ (LTO) NIMAM in distinct electrolyte solutions during lithium cycling. ${ }^{236}$ They demonstrated that not only a few fast cycles were required to qualify SEI on LTO anode but also to identify the best the electrolyte solution. More recently, in 2018, Kitz and co-workers have developed an operando EQCM-D with simultaneous in situ EIS to analyse the SEI formation on $\mathrm{Cu}$ layer as current collectors in LIBs. ${ }^{237}$ The evolution of mass and the mechanical properties of the SEI could be directly correlated to the electrode impedance. They concluded that this combination of in situ techniques provided key insights into the evolution of both the physical and electrochemical properties of layers deposited within the LIB during lithiation.
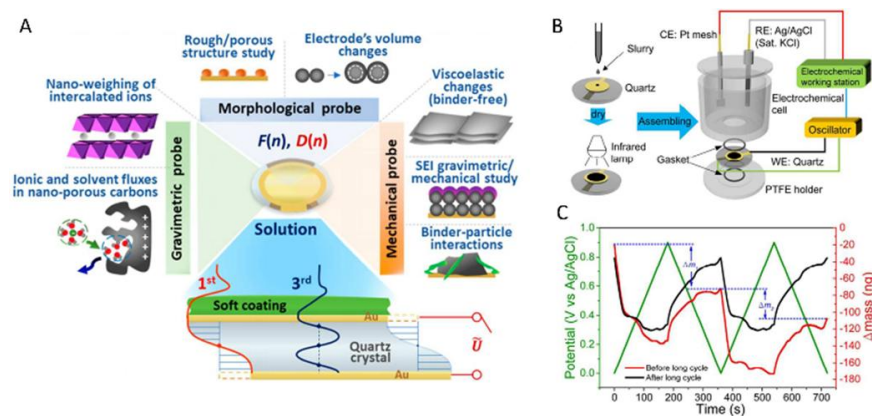

Figure 35. (A) Assembling process of the quartz electrode and construction of the electrochemical quartz crystal microbalance (EQCM) cell. The quartz electrode is placed between two gaskets to seal and to protect the quartz from scratching. (B) In situ EQCM results, mass change trend of the quartz electrode before (red) and after (black) long time cycling upon potential in a cyclic voltammetry cycling at a scan rate of $5 \mathrm{mV} \cdot \mathrm{s}^{-1}$. Reproduced with permission. ${ }^{238}$ Copyright 2017, Royal Society of Chemistry. (C) Gravimetric and non-gravimetric applications of EQCM-D for the characterization of energy-storage electrodes. (bottom panel) Acoustic waves for fundamental frequency and its 3rd overtone. Reproduced with permission. ${ }^{229}$ Copyright 2018, American Chemical Society.

For SIBs, Yao et al. used operando EQCM combined with Raman to find possible solutions towards the poor cycling stability of $\alpha-\mathrm{V}_{2} \mathrm{O}_{5}$ nanowires with a layered structure (Figure 35B,C). ${ }^{238}$ EQCM results showed a continuous mass loss that could be attributed to the slow dissolution of $\mathrm{V}_{2} \mathrm{O}_{5}$, whilst Raman data provided value information about the species involved in the energy storage process. The authors suggested the use of coating or additives in the electrolyte to improve the stability of this type of NIBAMs.

\subsubsection{Electrochemical dilatometry}

In situ electrochemical dilatometry (ECD) is the most suitable technique to investigate the effective height changes of composite electrodes, which has been firstly introduced in the late 1970s. ${ }^{239}$ In situ ECD integrates a displacement sensor within the cell to measure the vertical macroscopic displacement of the electrode to obtain the information related to volume expansion. ${ }^{240}$ In situ ECD has proved to provide important information regarding electrode deformation, electrointercalation of layered materials, and irreversible reaction, such as SEI formation. ${ }^{241} \mathrm{~A}$ combination of XRD and ECD techniques can most likely reveal the contribution of the microscopic crystal structural changes on the macroscopic expansion/shrinkage behavior of the whole composite electrode.

In the past about twenty years, in situ ECD has been widely applied to examine the electrochemical height electrode changes of various NIBAMs gradually. Actually, in situ ECD was usually utilized to investigate the effective changes of Si-based composite anodes due to large particle volume changes during lithiation/de-lithiation up to $400 \%{ }^{242-244}$ Park $^{245}$ and Hwang et al. ${ }^{246}$ studied the expansion of Sicontaining electrodes in the first charge/discharge cycle. Tranchot et al. investigated the long-term expansion and contraction behavior of Si-based composite electrodes for 45 cycles and found a continuous irreversible expansion of the electrode. ${ }^{240}$ Additionally, they reported a large deviation of $\pm 20 \%$ for the irreversible expansion in the first cycle from six individual measurements. Gomez-Camer ${ }^{247}$ and $\mathrm{Yu}$ et al. ${ }^{248}$ investigated the influence of the electrode binder on the expansion behavior of Si-containing electrodes for the first cycles. Gomez-Cramer et al. compared the expansion and contraction behavior of electrodes containing two different electrode binders, namely poly(vinylidene difluoride) (PVdF) and cross-linked sodiumcarboxymethyl cellulose/polyacrylic acid (CMC/PAA). The authors found that $\mathrm{CMC} / \mathrm{PAA}$ binder accommodates volume variations better than PVDF binder. However, it is indicated that the improved performance of CMC/PAA binder-based electrode is not resulted from the ability of inhibiting volume expansion of the binder but the high resistance to deformation attributed by its increased stiffness compared to PVDF (Figure 36a-d). ${ }^{247}$

Additionally, in situ ECD has been applied to explore other anode materials for LIBSs. Yang et al. showed ECD results on $\mathrm{Sn}$ and $\mathrm{SnSb}$ in 1997. ${ }^{251}$ They found dimensional changes of $\mathrm{SnSb}$, electrodes during cycling turned out to be quite small after the first insertion of lithium. By contrast, the 'breathing' of pure Sn was much more drastic. Wang et al. ${ }^{249}$ used in situ ECD combining with ex situ X-ray diffraction and ex situ Raman spectroscopy to study the interactions of $\mathrm{Sb}$ with $\mathrm{S}$ and $\mathrm{Se}$ and to reveal the reasons for the superior cycle stability of $\mathrm{Sb}_{2} \mathrm{Se}_{3}$ electrodes. They revealed that the thickness change of the $\mathrm{Sb}$ electrodes after lithiation to $660 \mathrm{mAh} \mathrm{g}^{-1}$ is about $110 \%$. After delithiation, there is still an electrode thickness change of $+40 \%$ compared to that of the initial $\mathrm{Sb}$ electrode (Figure 36e). This indicates 
that the electrode does not return to its original form after the removal of lithium. In contrast, the thickness changes are smaller for $\mathrm{Sb}_{2} \mathrm{~S}_{3}$ and even more so for the $\mathrm{Sb}_{2} \mathrm{Se}_{3}$ electrodes with a similar discharge capacity (Figure 36e). Specifically, $\mathrm{Sb}_{2} \mathrm{~S}_{3}$ shows a thickness change of $80 \%$ when it is fully lithiated to $850 \mathrm{mAh} \mathrm{g}^{-1}$, and the thickness change is about $+20 \%$ after it is fully charged to $3 \mathrm{~V}$. On the other hand, the $\mathrm{Sb}_{2} \mathrm{Se}_{3}$ electrode shows a thickness change of only around $50 \%$ after full lithiation and the thickness change is completely recovered after delithiation. The authors demonstrated that the improved mechanical properties of $\mathrm{Sb}_{2} \mathrm{Se}_{3}$ are ascribed to an efficient re-formation of the $\mathrm{Sb}_{2} \mathrm{Se}_{3}$ active material during charging, in line with their results of Raman spectroscopy and SEM. Zhang et al. ${ }^{252}$ used operando ECD analysis to investigate the Li metal|polymer electrolyte interface, and found the formation of "dead lithium" and cavities under an areal capacity utilization of $5 \mathrm{mAh} / \mathrm{cm}^{2}$, which was confirmed by post mortem surface analysis with SEM. It is also suggested by the cycling behavior of the $\mathrm{Li} \| \mathrm{Li}$ cell with high areal capacity utilization that lithium surface treatment is required to completely eliminate the formation of "dead lithium" and of cavities.
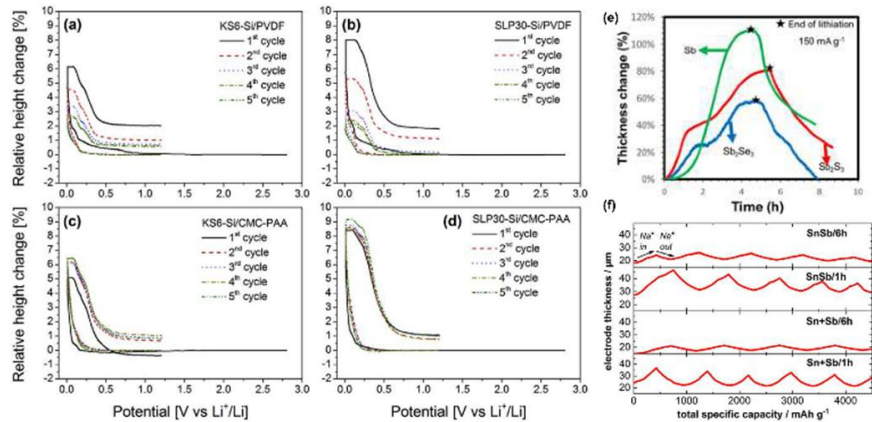

Figure 36. (a-d) Relative height change curves versus electrode potential of the first five cycles for KS6-Si electrodes with a) PVDF and c) CMC/PAA binders and SLP30-Si electrodes with b) PVDF and d) CMC/PAA binders Reproduced with permission. ${ }^{247}$ Copyright 2016, Elsevier. (e) Thickness change of the $\mathrm{Sb}, \mathrm{Sb}_{2} \mathrm{~S}_{3}$, and $\mathrm{Sb}_{2} \mathrm{Se}_{3}$ electrodes during the first lithiation and delithiation at a current rate of $150 \mathrm{~mA} \mathrm{~g}^{-1}$. Reproduced with permission ${ }^{249}$. Copyright 2019, American Chemical Society. (f) Electrode thicknesses change during sodiation/desodiation measured by ECD at $0.13 \mathrm{C}$. Reproduced with permission. ${ }^{250}$ Copyright 2019, Wiley.

For SIBs, Li et al. ${ }^{253}$ used in situ ECD to investigate the capacity fading in nanoporous Ge-based SIB anodes by measuring the corresponding (de)sodiation-induced strains. They found the capacity and strain amplitude exhibit the same decay trend during long-term cycling, which is expected since the capacity fades because of the volume changes arising during cycling. However, viewed from a different perspective, the similarity between capacity fading and strain decay suggests that one way to mitigate this capacity fading might be by preventing the initial strain from decaying, instead of getting rid of this strain. Furthermore, Brehm et al. ${ }^{250}$ applied in situ ECD to study the correlation of ball milling time ( $6 \mathrm{~h}$ vs. $1 \mathrm{~h}$ ) of $\mathrm{Sn}+\mathrm{Sb}$ and $\mathrm{Sn} / \mathrm{Sb}$ abodes for SIBs with their performances. They found that longer milling has only a minor influence on the initial capacity, but it significantly improves the cycle life stability because long milling times reduce the "breathing" of the electrodes during sodiation/desodiation although the electrodes are also more compact (Figure 36f).

\section{Conclusions and prospects}

The current tendency in the field is the development of NIBAMs with composite/hybrid structures, which combine materials with different composition, multidimensionality, porosity and redox features. Tremendous progress has been achieved for NIBAMs, but some serious problems such as capacity degradation, insufficient cycle stability, and safety issue have been hindering their large-scale particle applications. Although traditional electroanalytical methods are always very useful to characterize both quantitative and qualitative parameters of the whole battery (i.e. specific capacity, rate performance, capacity retention, coulombic efficiency, etc.), the development of advanced in situ/operando characterization techniques is key to acquire in-depth insights of the long-term evolution and durability of these NIBAMs into the battery without dissembling or even in operation. These techniques provide new approaches to study the physical and chemical characteristics of the NIBAMs, such as, the structure evolution, redox mechanism, SEI stability, dendrite formation, the impact of additives and nonaditives components, side reaction, and the interfacial interaction issue, constitute an essential knowledge required to evolve them, as well as their correlations with the electrochemical battery performance. Many remarkable discoveries have been made by in situ/operando characterization experiments during electrochemical process of batteries using NIBAMs. A comparison of various in situ/operando characterization techniques is listed in table 2 , including both their capability and shortcoming.

More concretely, in operando mode, X-ray techniques provide electronic and crystal structure information during the electrochemical processes carried out into the NIBAMs in cell batteries. Their major limitation is probable that synchrotron radiation facilities are usually required for a faster data acquisition at real time during the battery operation. In situ electron microscopy techniques offer high resolution images of the NIBAMs, from nano to atomic level, under battery operation conditions. In this case, the electrolyte composition is fundamental for an adequate image acquisition. In operando scanning probe microscopy techniques provide visual information about the interfacial structure of NIBAMs and its topography evolution during cycles. Their major limitation is the technical difficulties to allow the access of the physical probe into the battery cell, whilst maintaining the inert atmosphere required for the electrochemical measurements. In situ optical techniques allow relatively simple real-time monitoring of the chemical modification of NIBAM's surface into the batteries, mainly due to their fast data acquisition and high molecular specificity. The major inconveniences are the appropriate selection of cell batteries and electrolytes as well as the large amount of data analysis required. In addition to their higher penetration depth, in operando neutron techniques provide valuable information about structural changes, phases transitions, and the SEI formation during battery cycling, because neutrons are sensitive to the light elements and can easily distinguish isotopes. The major limitation could be associated with the difficulty of data analysis, linked to the photopeak estimation, nuclear reactions interference and neutron flux measurements. In situ magnetic techniques allow to probe the chemical environment in electrodes by monitoring paramagnetic species (e.g. ${ }^{7} \mathrm{Li}$ and ${ }^{23} \mathrm{Na}$ ), perceiving the 
structural changes over NIBAMs, electrolytes and their interfaces Probably, these are one of the most sensitive and useful technologies, in an operand configuration, for detecting dendrite growth. The limited materials for cell battery fabrication is one of the most important inconveniences. Other in operando techniques, such as, are becoming as interesting alternatives to the most established ones, providing punctual information of battery processes. In this case, an evident technical and knowledge evolution of these techniques is necessary yet.

Obviously, some techniques are still in the early development stage for characterization of NIBAMs, and even some ones haven't been applied to research NIBAMs. Therefore, there is considerable room to improve the state-of-the-art in situ/operando characterization techniques for NIBAM research. (i) It is vital to continue with the development of new analytical techniques under realistic battery assemblies and operation, which should be optimized to probe specific NIBAM physics and chemistries. (ii) Probably, in situ/operando developed for graphite anode and cathodes can be easily extended to exploit the NIBAMs. (iii) Furthermore, the integration of multiple tools can provide multidimension, multimodal, more reliable and valuable information about the insight of electrochemistry of NIBAMs. (iv) It is quite important to develop advanced techniques with the capacity to research the dynamics of NIBAMs in operation, which need to characterize the high-speed data in non-equilibrium. (v) The complex nature of the experimental set up together with the conditions of the battery cells should be taken into considerable attention because in situ/operando characterization methods require a substantial improvement of technical and technological facilities, especially when the perspective in the field is the combination of multiple advanced techniques simultaneously for the direct monitoring of composite/hybrid NIBAMs.

Table 2. Summary of advanced electroanalytical techniques reported in this review for direct monitoring and characterization of NIBAMs.

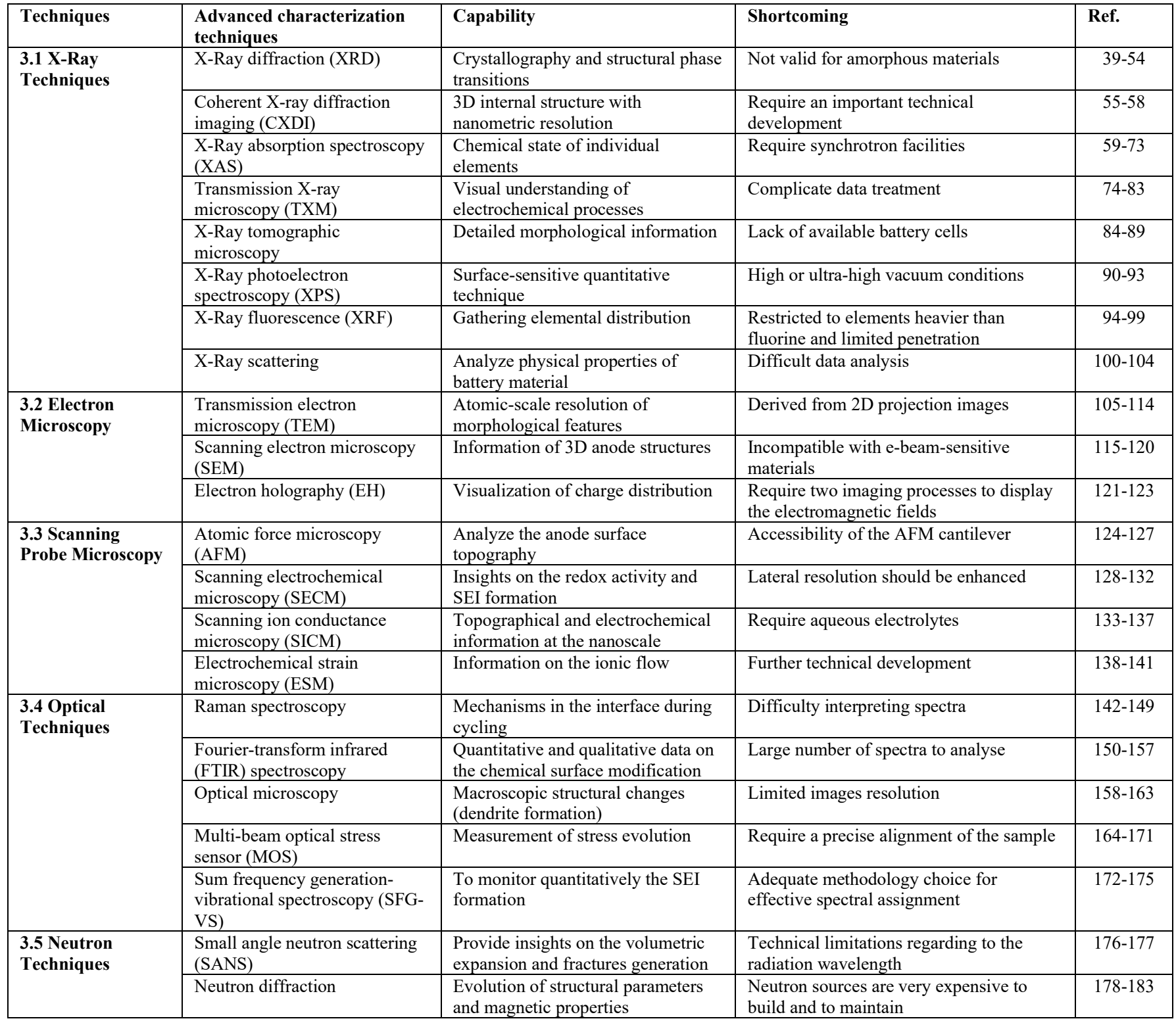




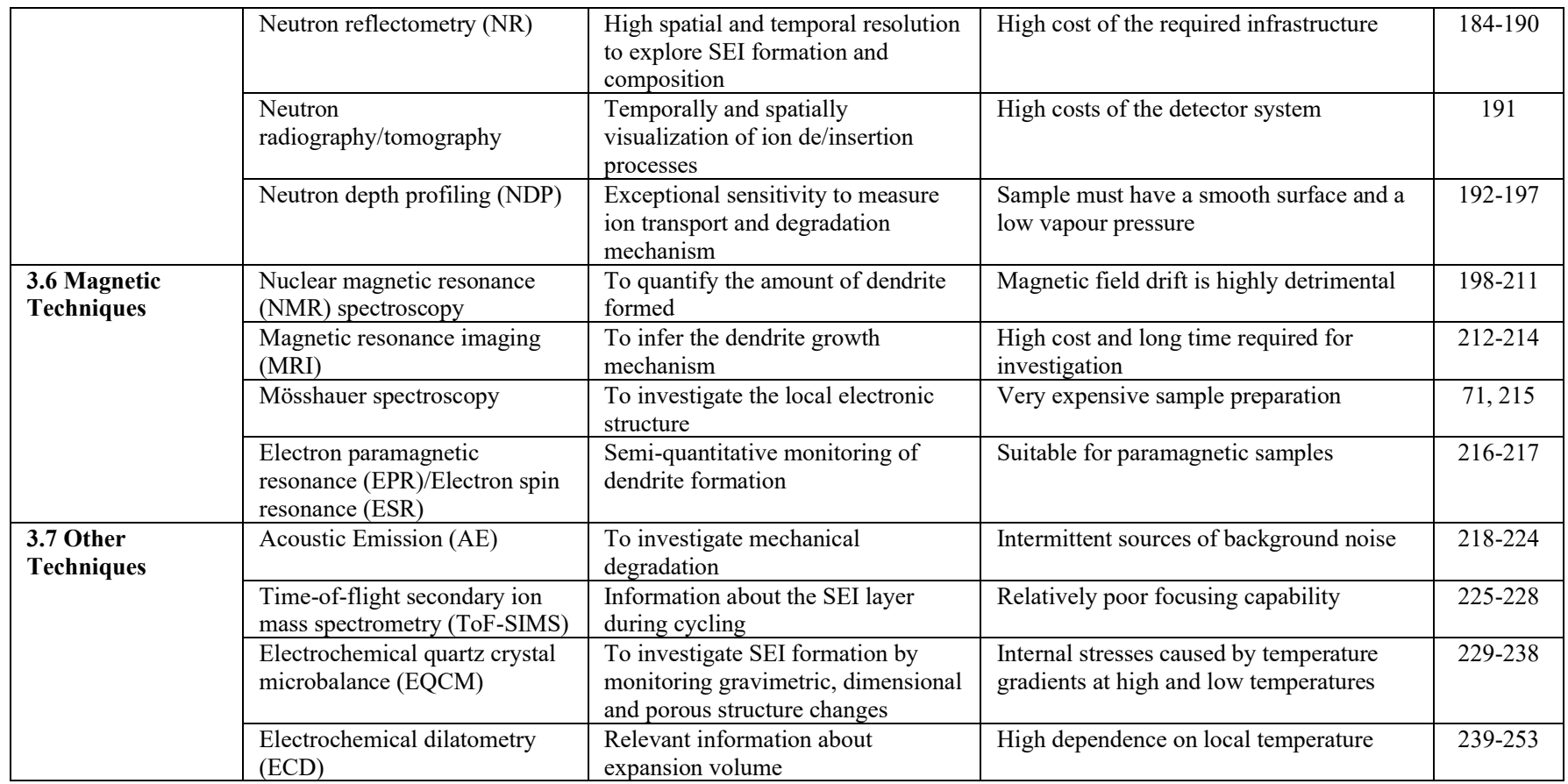

\section{Acknowledgements}

We apologize to authors whose work could not be included in this review due to space restrictions. J.M. Zhao and G.B Xu appreciate the The National Key Research and Development Program of China (No. 2016YFA0201300), the National Natural Science Foundation of China (No. 21675148 and 21874126), and K. C. Wong Education Foundation, Hong Kong. J.J.G.-C. acknowledges the Ministry of Economy and Competitiveness (MINECO) of Spain for a "Ramon y Cajal" contract (\#RyC-2014-14956), and for the MANA (CTQ20183961-R) and JEANS (CTQ2017-92264-EXP) projects, co-financed with European Regional Development Funds (FEDER). M.C. thanks FEDER and Andalusian Government (Consejería de Economía, Conocimiento, Empresasy Universidades, Junta de Andalucía) of Spain for the financial support through UCO-1263193 project. The publication has been prepared with support from RUDN University Program 5-100.

\section{References}

1. X. Zeng, M. Li, D. Abd El-Hady, W. Alshitari, A. S. Al-Bogami, J. Lu and K. Amine, Advanced Energy Materials, 2019, 9, 1900161.

2. K. Liu, K. Li, Q. Peng and C. Zhang, Frontiers of Mechanical Engineering, 2019, 14, 47-64.

3. Y. Liang, C.-Z. Zhao, H. Yuan, Y. Chen, W. Zhang, J.-Q. Huang, D. Yu, Y. Liu, M.-M. Titirici, Y.-L. Chueh, H. Yu and Q. Zhang, InfoMat, 2019, 1, 6-32.

4. Z. P. Cano, D. Banham, S. Ye, A. Hintennach, J. Lu, M. Fowler and Z. Chen, Nature Energy, 2018, 3, 279-289.

5. Y. Yang, S. Bremner, C. Menictas and M. Kay, Renewable and Sustainable Energy Reviews, 2018, 91, 109-125.

6. T. M. Gür, Energy \& Environmental Science, 2018, 11, 26962767.
7. D. Di Lecce, R. Verrelli and J. Hassoun, Green Chemistry, 2017, 19, 3442-3467.

8. Z. Shadike, E. Zhao, Y.-N. Zhou, X. Yu, Y. Yang, E. Hu, S. Bak, L. Gu and X.-Q. Yang, Advanced Energy Materials, 2018, 8, 1702588.

9. J.-Y. Hwang, S.-T. Myung and Y.-K. Sun, Chem. Soc. Rev., 2017, 46, 3529-3614.

10. X. Dou, I. Hasa, D. Saurel, C. Vaalma, L. Wu, D. Buchholz, D. Bresser, S. Komaba and S. Passerini, Mater. Today, 2019, 23, 87-104.

11. F. Li, Z. Wei, A. Manthiram, Y. Feng, J. Ma and L. Mai, Journal of Materials Chemistry A, 2019, 7, 9406-9431.

12. R. Rajagopalan, Y. Tang, X. Ji, C. Jia and H. Wang, Adv. Funct. Mater., 2020, 30, 1909486.

13. S. Guo, J. Yi, Y. Sun and H. Zhou, Energy \& Environmental Science, 2016, 9, 2978-3006.

14. W. Qi, J. G. Shapter, Q. Wu, T. Yin, G. Gao and D. Cui, Journal of Materials Chemistry A, 2017, 5, 19521-19540.

15. N. Mahmood, T. Tang and Y. Hou, Advanced Energy Materials, 2016, 6, 1600374.

16. G. Li, B. Huang, Z. Pan, X. Su, Z. Shao and L. An, Energy \& Environmental Science, 2019, 12, 2030-2053.

17. L. Zhang, S. Peng, Y. Ding, X. Guo, Y. Qian, H. Celio, G. He and G. Yu, Energy \& Environmental Science, 2019, 12, 19891998.

18. B. Chen, D. Chao, E. Liu, M. Jaroniec, N. Zhao and S.-Z. Qiao, Energy \& Environmental Science, 2020, DOI: 10.1039/C9EE03549D.

19. R. Zhao, H. Di, X. Hui, D. Zhao, R. Wang, C. Wang and L. Yin, Energy \& Environmental Science, 2020, 13, 246-257.

20. L. Li, Y. Zheng, S. Zhang, J. Yang, Z. Shao and Z. Guo, Energy \& Environmental Science, 2018, 11, 2310-2340.

21. Y. Yan, C. Cheng, L. Zhang, Y. Li and J. Lu, Advanced Energy Materials, 2019, 9, 1900148.

22. A. D. Robertson, H. Tukamoto and J. T. S. Irvine, J. Electrochem. Soc., 1999, 146, 3958-3962.

23. G. Lin, H. Wang, L. Zhang, Q. Cheng, Z. Gong and K. Ostrikov, J. Power Sources, 2019, 437, 226909. 
24.

F. Xie, L. Zhang, D. Su, M. Jaroniec and S. Z. Qiao, Adv. Mater., 2017, n/a-n/a.

25. C. Zeng, F. Xie, X. Yang, M. Jaroniec, L. Zhang and S.-Z. Qiao, Angew. Chem. Int. Ed., 2018, 57, 8540-8544.

26. D.-I. Stroe, M. Swierczynski, A.-I. Stroe and S. Knudsen Kær, Batteries, 2016, 2.

27.

28.

29

J. Guo, A. Sun, X. Chen, C. Wang and A. Manivannan, Electrochim. Acta, 2011, 56, 3981-3987.

H. Li, X. Huang and L. Chen, J. Power Sources, 1999, 81-82, 340-345.

30. H. Schranzhofer, J. Bugajski, H. J. Santner, C. Korepp, K. C. Möller, J. O. Besenhard, M. Winter and W. Sitte, J. Power Sources, 2006, 153, 391-395.

31. J. Guo, A. Sun, X. Chen, C. Wang and A. Manivannan, Electrochim. Acta, 2011, 56, 3981-3987.

32.

M. V. Reddy, G. Prithvi, K. P. Loh and B. V. R. Chowdari, ACS Applied Materials \& Interfaces, 2014, 6, 680-690.

33. E. Radvanyi, K. Van Havenbergh, W. Porcher, S. Jouanneau, J.-S. Bridel, S. Put and S. Franger, Electrochim. Acta, 2014, 137, 751-757.

34. J. Mao, D. Niu, N. Zheng, G. Jiang, W. Zhao, J. Shi and Y. Li, ACS Sustainable Chemistry \& Engineering, 2019, 7, 34243433.

35. L. Hu, C. Shang, L. Huang, X. Wang and G. Zhou, lonics, 2019, DOI: 10.1007/s11581-019-03230-y.

36. L. Guo, L. Cao, J. Huang, Y. Wang, W. Li, H. Qi, S. Chen and J. Li, J. Alloys Compd., 2019, 810, 151930.

37. W.-C. Chang, J.-H. Wu, K.-T. Chen and H.-Y. Tuan, Advanced Science, 2019, 6, 1801354.

38. H. Wang, D. Yu, X. Wang, Z. Niu, M. Chen, L. Cheng, W. Zhou and L. Guo, Angew. Chem., $\mathbf{0}$.

39. S. N. S. Hapuarachchi, Z. Sun and C. Yan, Advanced Sustainable Systems, 2018, 2, 1700182.

40.

L. C. Loaiza, E. Salager, N. Louvain, A. Boulaoued, A. ladecola, P. Johansson, L. Stievano, V. Seznec and L. Monconduit, Journal of Materials Chemistry A, 2017, 5, 12462-12473.

41. T. D. Hatchard and J. R. Dahn, J. Electrochem. Soc., 2004, 151, A838-A842.

42.

J. Li and J. R. Dahn, J. Electrochem. Soc., 2007, 154, A156A161.

43. S. Misra, N. Liu, J. Nelson, S. S. Hong, Y. Cui and M. F. Toney, ACS Nano, 2012, 6, 5465-5473.

44. M. P. B. Glazer, J. Cho, J. Almer, J. Okasinski, P. V. Braun and D. C. Dunand, Advanced Energy Materials, 2015, 5, 1500466.

45. P. Bach, I. Valencia-Jaime, U. Rütt, O. Gutowski, A. H. Romero and F. U. Renner, Chem. Mater., 2016, 28, 29412948.

46. F. J. Q. Cortes, M. G. Boebinger, M. Xu, A. Ulvestad and M. T. McDowell, ACS Energy Letters, 2018, 3, 349-355.

47. Y. Dong, Y. Ma, D. Li, Y. Liu, W. Chen, X. Feng and J. Zhang, Chemical Science, 2018, 9, 8682-8691.

48. X. Yu, H. Pan, W. Wan, C. Ma, J. Bai, Q. Meng, S. N. Ehrlich, Y.-S. Hu and X.-Q. Yang, Nano Lett., 2013, 13, 4721-4727.

49. S. Hartung, N. Bucher, J. B. Franklin, A. M. Wise, L. Y. Lim, H.-Y. Chen, J. N. Weker, M.-E. Michel-Beyerle, M. F. Toney and M. Srinivasan, Advanced Energy Materials, 2016, 6, 1502336.

50. H. Gao, W. Ma, W. Yang, J. Wang, J. Niu, F. Luo, Z. Peng and Z. Zhang, J. Power Sources, 2018, 379, 1-9.
51.

H. Gao, L. Song, J. Niu, C. Zhang, T. Kou, Y. Sun, J. Qin, Z. Peng and Z. Zhang, Journal of Materials Chemistry A, 2019, 7, 13602-13613.

52. F. Xie, L. Zhang, Q. Gu, D. Chao, M. Jaroniec and S.-Z. Qiao, Nano Energy, 2019, 60, 591-599.

53. Y. Liu, Z. Tai, J. Zhang, W. K. Pang, Q. Zhang, H. Feng, K. Konstantinov, Z. Guo and H. K. Liu, Nature Communications, 2018, 9, 3645.

54. F. Xie, L. Zhang, B. Chen, D. Chao, Q. Gu, B. Johannessen, M. Jaroniec and S.-Z. Qiao, Matter, 2019, DOI: https://doi.org/10.1016/i.matt.2019.07.006.

55. M. Wolf, B. M. May and J. Cabana, Chem. Mater., 2017, 29, 3347-3362.

56. Y. Gao, R. Harder, S. H. Southworth, J. R. Guest, X. Huang, Z. Yan, L. E. Ocola, Y. Yifat, N. Sule, P. J. Ho, M. Pelton, N. F. Scherer and L. Young, Proceedings of the National Academy of Sciences, 2019, 116, 4018.

57. M. J. Cherukara, R. Pokharel, T. S. O'Leary, J. K. Baldwin, E. Maxey, W. Cha, J. Maser, R. J. Harder, S. J. Fensin and R. L. Sandberg, Nature Communications, 2018, 9, 3776.

58. A. Ulvestad, A. Singer, H.-M. Cho, J. N. Clark, R. Harder, J. Maser, Y. S. Meng and O. G. Shpyrko, Nano Lett., 2014, 14, 5123-5127.

59. Q. Gu, J. A. Kimpton, H. E. A. Brand, Z. Wang and S. Chou, Advanced Energy Materials, 2017, 7, 1602831.

60. L. Y. Lim, S. Fan, H. H. Hng and M. F. Toney, The Journal of Physical Chemistry C, 2015, 119, 22772-22777.

61. S. Permien, S. Indris, A.-L. Hansen, M. Scheuermann, D. Zahn, U. Schürmann, G. Neubüser, L. Kienle, E. Yegudin and W. Bensch, ACS Applied Materials \& Interfaces, 2016, 8, 15320-15332.

62. S. Permien, S. Indris, G. Neubüser, A. Fiedler, L. Kienle, S. Zander, S. Doyle, B. Richter and W. Bensch, Chemistry - A European Journal, 2016, 22, 16929-16938.

63.

D. Muller-Bouvet, N. Emery, N. Tassali, E. Panabière, S. Bach, O. Crosnier, T. Brousse, C. Cénac-Morthe, A. Michalowicz and J. P. Pereira-Ramos, PCCP, 2017, 19, 27204-27211.

64. M. Fehse, A. Iadecola, M. T. Sougrati, P. Conti, M. Giorgetti and L. Stievano, Energy Storage Materials, 2019, 18, 328337.

65. M. Fehse, A. Darwiche, M. T. Sougrati, E. M. Kelder, A. V. Chadwick, M. Alfredsson, L. Monconduit and L. Stievano, Chem. Mater., 2017, 29, 10446-10454.

66. S. Schmidt, D. Sheptyakov, J.-C. Jumas, M. Medarde, P. Benedek, P. Novák, S. Sallard and C. Villevieille, Chem. Mater., 2015, 27, 7889-7895.

67. S. Schmidt, S. Sallard, C. Borca, T. Huthwelker, P. Novák and C. Villevieille, Chem. Commun., 2018, 54, 4939-4942.

68. S.-H. Yu, X. Huang, J. D. Brock and H. D. Abruña, J. Am. Chem. Soc., 2019, 141, 8441-8449.

69. A. Darwiche, F. Murgia, M. Fehse, A. Mahmoud, A. ladecola, S. Belin, C. La Fontaine, V. Briois, R. P. Hermann, B. Fraisse, R. Berthelot, M. T. Sougrati, L. Monconduit and L. Stievano, Energy Storage Materials, 2019, 21, 1-13.

70. M. Fehse, M. T. Sougrati, A. Darwiche, V. Gabaudan, C. La Fontaine, L. Monconduit and L. Stievano, Journal of Materials Chemistry A, 2018, 6, 8724-8734.

71. M. Fehse, D. Bessas, A. Darwiche, A. Mahmoud, G. Rahamim, C. La Fontaine, R. P. Hermann, D. Zitoun, L. Monconduit, L. Stievano and M. T. Sougrati, Batteries \& Supercaps, 2019, 2, 66-73. 
J. Ni, W. Wang, C. Wu, H. Liang, J. Maier, Y. Yu and L. Li, Adv. Mater., 2017, 29, 1605607.

73. D. Dixon, M. Ávila, H. Ehrenberg and A. Bhaskar, ACS Omega, 2019, 4, 9731-9738.

74. N.-H. Yang, Y.-F. Song and R.-S. Liu, ACS Energy Letters, 2018, 3, 1911-1928.

75. Y. Wu and N. Liu, Chem, 2018, 4, 438-465

76. T. Li, X. Zhou, Y. Cui, C. Lim, H. Kang, B. Yan, J. Wang, J. Wang, Y. Fu and L. Zhu, Electrochim. Acta, 2019, 314, 212218.

77. S.-C. Chao, Y.-C. Yen, Y.-F. Song, Y.-M. Chen, H.-C. Wu and N.-L. Wu, Electrochem. Commun., 2010, 12, 234-237.

78. S.-C. Chao, Y.-C. Yen, Y.-F. Song, H.-S. Sheu, H.-C. Wu and N.-L. Wu, J. Electrochem. Soc., 2011, 158, A1335-A1339.

79. S.-C. Chao, Y.-F. Song, C.-C. Wang, H.-S. Sheu, H.-C. Wu and N.-L. Wu, The Journal of Physical Chemistry C, 2011, 115, 22040-22047.

80. F. Meirer, J. Cabana, Y. Liu, A. Mehta, J. C. Andrews and P. Pianetta, Journal of Synchrotron Radiation, 2011, 18, 773 781.

81. J. N. Weker, N. Liu, S. Misra, J. C. Andrews, Y. Cui and M. F. Toney, Energy \& Environmental Science, 2014, 7, 27712777.

82. J. B. Cook, T. C. Lin, E. Detsi, J. N. Weker and S. H. Tolbert, Nano Lett., 2017, 17, 870-877.

83. J.-H. Cheng, A. A. Assegie, C.-J. Huang, M.-H. Lin, A. M. Tripathi, C.-C. Wang, M.-T. Tang, Y.-F. Song, W.-N. Su and B. J. Hwang, The Journal of Physical Chemistry C, 2017, 121, 7761-7766.

84. M. Ebner, F. Marone, M. Stampanoni and V. Wood, Science, 2013, 342, 716.

85. J. Wang, Y.-c. K. Chen-Wiegart and J. Wang, Angew. Chem. Int. Ed., 2014, 53, 4460-4464.

86. F. Tariq, V. Yufit, D. S. Eastwood, Y. Merla, M. Biton, B. Wu, Z. Chen, K. Freedman, G. Offer, E. Peled, P. D. Lee, D. Golodnitsky and N. Brandon, ECS Electrochemistry Letters, 2014, 3, A76-A78.

87. V. Vanpeene, A. Etiemble, A. Bonnin, E. Maire and L. Roué, J. Power Sources, 2017, 350, 18-27.

88. J. Tippens, J. C. Miers, A. Afshar, J. A. Lewis, F. J. Q. Cortes, H. Qiao, T. S. Marchese, C. V. Di Leo, C. Saldana and M. T. McDowell, ACS Energy Letters, 2019, 4, 1475-1483.

89. D. P. Finegan, A. Vamvakeros, L. Cao, C. Tan, T. M. M. Heenan, S. R. Daemi, S. D. M. Jacques, A. M. Beale, M. Di Michiel, K. Smith, D. J. L. Brett, P. R. Shearing and C. Ban, Nano Lett., 2019, 19, 3811-3820.

90. K. N. Wood and G. Teeter, ACS Applied Energy Materials, 2018, 1, 4493-4504.

91. C.-Y. Tang, R. T. Haasch and S. J. Dillon, Chem. Commun., 2016, 52, 13257-13260.

92. K. N. Wood, K. X. Steirer, S. E. Hafner, C. Ban, S. Santhanagopalan, S.-H. Lee and G. Teeter, Nature Communications, 2018, 9, 2490.

93. L. Madec, J.-B. Ledeuil, G. Coquil, L. Monconduit and H. Martinez, ACS Applied Energy Materials, 2019, 2, 53005307.

94. Y. Terada, Y. Nishiwaki, I. Nakai and F. Nishikawa, J. Power Sources, 2001, 97-98, 420-422.

95. R. Robert, D. Zeng, A. Lanzirotti, P. Adamson, S. J. Clarke and C. P. Grey, Chem. Mater., 2012, 24, 2684-2691.

96. K. Yamamoto, T. Minato, S. Mori, D. Takamatsu, Y. Orikasa, H. Tanida, K. Nakanishi, H. Murayama, T. Masese, T. Mori,
H. Arai, Y. Koyama, Z. Ogumi and Y. Uchimoto, The Journal of Physical Chemistry C, 2014, 118, 9538-9543.

97. Y. Li, J. N. Weker, W. E. Gent, D. N. Mueller, J. Lim, D. A. Cogswell, T. Tyliszczak and W. C. Chueh, Adv. Funct. Mater., 2015, 25, 3677-3687.

98. U. Boesenberg, M. Falk, C. G. Ryan, R. Kirkham, M. Menzel, J. Janek, M. Fröba, G. Falkenberg and U. E. A. Fittschen, Chem. Mater., 2015, 27, 2525-2531.

99. J. L. Durham, A. B. Brady, C. A. Cama, D. C. Bock, C. J. Pelliccione, Q. Zhang, M. Ge, Y. R. Li, Y. Zhang, H. Yan, X. Huang, Y. Chu, E. S. Takeuchi, K. J. Takeuchi and A. C. Marschilok, PCCP, 2017, 19, 22329-22343.

100. M. P. B. Glazer, J. S. Okasinski, J. D. Almer and Y. Ren, MRS Bull., 2016, 41, 460-465.

101. K. E. Silberstein, M. A. Lowe, B. Richards, J. Gao, T. Hanrath and H. D. Abruña, Langmuir, 2015, 31, 2028-2035.

102. S. M. Bhaway, Z. Qiang, Y. Xia, X. Xia, B. Lee, K. G. Yager, L. Zhang, K. Kisslinger, Y.-M. Chen, K. Liu, Y. Zhu and B. D. Vogt, ACS Nano, 2017, 11, 1443-1454.

103. G. E. Möhl, E. Metwalli and P. Müller-Buschbaum, ACS Energy Letters, 2018, 3, 1525-1530.

104. C. L. Berhaut, D. Z. Dominguez, P. Kumar, P.-H. Jouneau, W. Porcher, D. Aradilla, S. Tardif, S. Pouget and S. Lyonnard, ACS Nano, 2019, DOI: 10.1021/acsnano.9b05055.

105. X. H. Liu and J. Y. Huang, Energy \& Environmental Science, 2011, 4, 3844-3860.

106. M. T. McDowell, S. W. Lee, J. T. Harris, B. A. Korgel, C. Wang, W. D. Nix and Y. Cui, Nano Lett., 2013, 13, 758-764.

107. M. Gu, L. R. Parent, B. L. Mehdi, R. R. Unocic, M. T. McDowell, R. L. Sacci, W. Xu, J. G. Connell, P. Xu, P. Abellan, X. Chen, Y. Zhang, D. E. Perea, J. E. Evans, L. J. Lauhon, J.-G. Zhang, J. Liu, N. D. Browning, Y. Cui, I. Arslan and C.-M. Wang, Nano Lett., 2013, 13, 6106-6112.

108. Z.-L. Xu, B. Zhang, Y. Gang, K. Cao, M. A. Garakani, S. Abouali, J. Huang, J.-Q. Huang, E. K. Heidari, H. Wang and J.-K. Kim, Energy Storage Materials, 2015, 1, 25-34.

109. Q. Su, S. Wang, L. Yao, H. Li, G. Du, H. Ye and Y. Fang, Scientific Reports, 2016, 6, 28197.

110. P. Gao, L. Wang, Y.-Y. Zhang, Y. Huang, L. Liao, P. Sutter, K. Liu, D. Yu and E.-G. Wang, Nano Lett., 2016, 16, 5582-5588. K. He, Z. Yao, S. Hwang, N. Li, K. Sun, H. Gan, Y. Du, H. Zhang, C. Wolverton and D. Su, Nano Lett., 2017, 17, 57265733.

112. K. Gnanasekaran, G. d. With and H. Friedrich, Royal Society Open Science, 2018, 5, 171838.

113. Y. Huang, C. Zhu, S. Zhang, X. Hu, K. Zhang, W. Zhou, S. Guo, F. Xu and H. Zeng, Nano Lett., 2019, 19, 1118-1123.

114. X. Wang, Z. Yao, S. Hwang, Y. Pan, H. Dong, M. Fu, N. Li, K. Sun, H. Gan, Y. Yao, A. Aspuru-Guzik, Q. Xu and D. Su, ACS Nano, 2019, 13, 9421-9430.

115. C.-Y. Chen, T. Sano, T. Tsuda, K. Ui, Y. Oshima, M. Yamagata, M. Ishikawa, M. Haruta, T. Doi, M. Inaba and S. Kuwabata, Scientific Reports, 2016, 6, 36153.

116. C.-Y. Chen, A. Sawamura, T. Tsuda, S. Uchida, M. Ishikawa and S. Kuwabata, ACS Applied Materials \& Interfaces, 2017, 9, 35511-35515.

117. J. Duan, Y. Zheng, W. Luo, W. Wu, T. Wang, Y. Xie, S. Li, J. Li and Y. Huang, National Science Review, 2020, DOI: 10.1093/nsr/nwz222.

118. J.-Y. Hwang, S.-J. Park, C. S. Yoon and Y.-K. Sun, Energy \& Environmental Science, 2019, 12, 2174-2184. 
119. D. J. Miller, C. Proff, J. G. Wen, D. P. Abraham and J. Bareño, Advanced Energy Materials, 2013, 3, 1098-1103.

120. X. Zhou, T. Li, Y. Cui, Y. Fu, Y. Liu and L. Zhu, ACS Applied Materials \& Interfaces, 2019, 11, 1733-1738.

121. K. Yamamoto, Y. Iriyama and T. Hirayama, Microscopy, 2016, 66, 50-61.

122. Z. Gan, M. Gu, J. Tang, C.-Y. Wang, Y. He, K. L. Wang, C. Wang, D. J. Smith and M. R. McCartney, Nano Lett., 2016, 16, 3748-3753.

123. Y. Wen, X. Chen, X. Lu and L. Gu, Journal of Energy Chemistry, 2018, 27, 1397-1401.

124. B. Breitung, P. Baumann, H. Sommer, J. Janek and T. Brezesinski, Nanoscale, 2016, 8, 14048-14056.

125. I. Yoon, D. P. Abraham, B. L. Lucht, A. F. Bower and P. R. Guduru, Advanced Energy Materials, 2016, 6, 1600099.

126. S. D. Lacey, J. Wan, A. v. W. Cresce, S. M. Russell, J. Dai, W. Bao, K. Xu and L. Hu, Nano Lett., 2015, 15, 1018-1024.

127. M. Han, C. Zhu, Q. Zhao, C. Chen, Z. Tao, W. Xie, F. Cheng and J. Chen, ACS Applied Materials \& Interfaces, 2017, 9, 28620-28626.

128. P. Bertoncello, Energy \& Environmental Science, 2010, 3, 1620-1633.

129. E. Ventosa and W. Schuhmann, PCCP, 2015, 17, 2844128450.

130. G. Zampardi, E. Ventosa, F. La Mantia and W. Schuhmann, Chem. Commun., 2013, 49, 9347-9349.

131. G. Zampardi, E. Ventosa, F. La Mantia and W. Schuhmann, Electroanalysis, 2015, 27, 1017-1025.

132. D. Liu, Q. Yu, S. Liu, K. Qian, S. Wang, W. Sun, X.-Q. Yang, F. Kang and B. Li, Journal of Physical Chemistry C, 2019, 123, 12797-12806.

133. Z. J. Barton and J. Rodriguez-Lopez, Analytical and Bioanalytical Chemistry, 2016, 408, 2707-2715.

134. J. Rheinlaender and T. E. Schäffer, J. Appl. Phys., 2009, 105, 094905.

135. A. I. Shevchuk, G. I. Frolenkov, D. Sánchez, P. S. James, N. Freedman, M. J. Lab, R. Jones, D. Klenerman and Y. E. Korchev, Angew. Chem. Int. Ed., 2006, 45, 2212-2216.

136. A. L. Lipson, R. S. Ginder and M. C. Hersam, Adv. Mater., 2011, 23, 5613-5617.

137. A. L. Lipson, K. Puntambekar, D. J. Comstock, X. Meng, M. L. Geier, J. W. Elam and M. C. Hersam, Chem. Mater., 2014, 26, 935-940.

138. N. Balke, S. Jesse, Y. Kim, L. Adamczyk, A. Tselev, I. N. Ivanov, N. J. Dudney and S. V. Kalinin, Nano Lett., 2010, 10, 3420-3425.

139. K. Romanyuk, C. M. Costa, S. Y. Luchkin, A. L. Kholkin and S. Lanceros-Mendez, Langmuir, 2016, 32, 5267-5276.

140. N. Balke, S. Jesse, Y. Kim, L. Adamczyk, I. N. Ivanov, N. J. Dudney and S. V. Kalinin, ACS Nano, 2010, 4, 7349-7357.

141. S. Jesse, N. Balke, E. Eliseev, A. Tselev, N. J. Dudney, A. N. Morozovska and S. V. Kalinin, Acs Nano, 2011, 5, $9682-$ 9695.

142. D. Liu, Z. Shadike, R. Lin, K. Qian, H. Li, K. Li, S. Wang, Q. Yu, M. Liu, S. Ganapathy, X. Qin, Q.-H. Yang, M. Wagemaker, F. Kang, X.-Q. Yang and B. Li, Adv. Mater., 2019, 31, 1806620.

143. C. M. Julien and A. Mauger.

144. Z. Zeng, N. Liu, Q. Zeng, S. W. Lee, W. L. Mao and Y. Cui, Nano Energy, 2016, 22, 105-110.

145. M. Jana and R. N. Singh, Appl. Phys. Lett., 2017, 111, 063901.
146.

147.

Hardwick, Chem. Commun., 2016, 52, 3970-3973.

S. Tardif, E. Pavlenko, L. Quazuguel, M. Boniface, M. Maréchal, J.-S. Micha, L. Gonon, V. Mareau, G. Gebel, P. Bayle-Guillemaud, F. Rieutord and S. Lyonnard, ACS Nano, 2017, 11, 11306-11316.

148. A. Krause, O. Tkacheva, A. Omar, U. Langklotz, L. Giebeler, S. Dörfler, F. Fauth, T. Mikolajick and W. M. Weber, J. Electrochem. Soc., 2019, 166, A5378-A5385.

149. K. Li, J. Zhang, D. Lin, D.-W. Wang, B. Li, W. Lv, S. Sun, Y.-B. He, F. Kang, Q.-H. Yang, L. Zhou and T.-Y. Zhang, Nature Communications, 2019, 10, 725.

150. F. Shi, P. N. Ross, G. A. Somorjai and K. Komvopoulos, The Journal of Physical Chemistry C, 2017, 121, 14476-14483.

151. C. Marino, A. Boulaoued, J. Fullenwarth, D. Maurin, N. Louvain, J.-L. Bantignies, L. Stievano and L. Monconduit, The Journal of Physical Chemistry C, 2017, 121, 2659826606.

152. D. Alves Dalla Corte, G. Caillon, C. Jordy, J.-N. Chazalviel, M. Rosso and F. Ozanam, Advanced Energy Materials, 2016, 6, 1501768.

153. B. M. Koo, D. A. D. Corte, J.-N. Chazalviel, F. Maroun, M. Rosso and F. Ozanam, Advanced Energy Materials, 2018, 8, 1702568.

154. Y. B. Yohannes, S. D. Lin and N.-L. Wu, J. Electrochem. Soc., 2017, 164, A3641-A3648.

155. Y. Horowitz, H.-L. Han, W. T. Ralston, J. R. de Araujo, E. Kreidler, C. Brooks and G. A. Somorjai, Advanced Energy Materials, 2017, 7, 1602060.

156. Y. Horowitz, H.-L. Han, F. A. Soto, W. T. Ralston, P. B. Balbuena and G. A. Somorjai, Nano Lett., 2018, 18, 11451151.

157. J. Z. Olson, P. K. Johansson, D. G. Castner and C. W. Schlenker, Chem. Mater., 2018, 30, 1239-1248.

158. L. Y. Beaulieu, T. D. Hatchard, A. Bonakdarpour, M. D. Fleischauer and J. R. Dahn, J. Electrochem. Soc., 2003, 150, A1457-A1464.

159. J. Duay, K. W. Schroder, S. Murugesan and K. J. Stevenson, Acs Applied Materials \& Interfaces, 2016, 8, 17642-17650.

160. Y. Feng, N. Thuy-Doan-Trang, M. Panagopoulou, A. Cheriet, B. M. Koo, C. Henry-de-Villeneuve, M. Rosso and F. Ozanam, Electrochim. Acta, 2019, 302, 249-258.

161. A. Timmons and J. R. Dahn, J. Electrochem. Soc., 2007, 154, A444-A448.

162. S. Bhattacharya and A. T. Alpas, J. Power Sources, 2016, 328, 300-310.

163. W. Manalastas, Jr., J. Rikarte, R. J. Chater, R. Brugge, A. Aguadero, L. Buannic, A. Llordes, F. Aguesse and J. Kilner, J. Power Sources, 2019, 412, 287-293.

164. V. A. Sethuraman, M. J. Chon, M. Shimshak, V. Srinivasan and P. R. Guduru, J. Power Sources, 2010, 195, 5062-5066.

165. V. A. Sethuraman, M. J. Chon, M. Shimshak, N. Van Winkle and P. R. Guduru, Electrochem. Commun., 2010, 12, 16141617.

166. J. Chen, L. Yang, Y. Han, Y.-H. Bao, K.-L. Zhang, X. Li, J. Pang, H.-S. Chen, W.-L. Song, Y.-J. Wei and D.-N. Fang, J. Power Sources, 2019, 444, 227227.

167. A. Mukhopadhyay, R. Kali, S. Badjate, A. Tokranov and B. W. Sheldon, Scripta Mater., 2014, 92, 47-50.

V. A. P. Kali R., Badjate S., Mitra S., Bhandakkar T. and Mukhopadhyay A. , Meeting Abstracts, 2016, MA2016-01, 388. 
169. M. Pharr, Y. S. Choi, D. Lee, K. H. Oh and J. J. Vlassak, J. 196. Power Sources, 2016, 304, 164-169.

170. A. Al-Obeidi, D. Kramer, C. V. Thompson and R. Mönig, J. Power Sources, 2015, 297, 472-480.

171. Z. Choi, D. Kramer and R. Mönig, J. Power Sources, 2013, 240, 245-251.

172. P. Mukherjee, A. Lagutchev and D. D. Dlott, J. Electrochem. Soc., 2012, 159, A244-A252.

173. Y. Horowitz, H.-L. Han and G. A. Somorjai, Industrial \& Engineering Chemistry Research, 2018, 57, 1480-1486.

174. Y. Horowitz, H.-G. Steinrück, H.-L. Han, C. Cao, I. I. Abate, Y. Tsao, M. F. Toney and G. A. Somorjai, Nano Lett., 2018, 18, 2105-2111.

175. A. M. Tripathi, W.-N. Su and B. J. Hwang, Chem. Soc. Rev., 2018, 47, 736-851.

176. M. J. Hollamby, PCCP, 2013, 15, 10566-10579.

177. X. He, R. Wang, M. C. Stan, E. Paillard, J. Wang, H. Frielinghaus and J. Li, Advanced Materials Interfaces, 2017, 4.

178. J.-F. Colin, V. Godbole and P. Novák, Electrochem. Commun., 2010, 12, 804-807.

179. W. K. Pang, N. Sharma, V. K. Peterson, J.-J. Shiu and S.-h. Wu, J. Power Sources, 2014, 246, 464-472.

180. W. K. Pang, V. K. Peterson, N. Sharma, J.-J. Shiu and S.-h. Wu, Chem. Mater., 2014, 26, 2318-2326.

181. W. K. Pang, V. K. Peterson, N. Sharma, J.-J. Shiu and S.-h. Wu, Powder Diffr., 2014, 29, S59-S63.

182. G. Du, N. Sharma, V. K. Peterson, J. A. Kimpton, D. Jia and Z. Guo, Adv. Funct. Mater., 2011, 21, 3990-3997.

183. N. Sharma, G. Du, A. J. Studer, Z. Guo and V. K. Peterson, Solid State lonics, 2011, 199, 37-43.

184. J. E. Owejan, J. P. Owejan, S. C. DeCaluwe and J. A. Dura, Chem. Mater., 2012, 24, 2133-2140.

185. B. Jerliu, L. Doerrer, E. Hueger, G. Borchardt, R. Steitz, U. Geckle, V. Oberst, M. Bruns, O. Schneider and H. Schmidt, PCCP, 2013, 15, 7777-7784.

186. B. Jerliu, E. Hüger, L. Dörrer, B. K. Seidlhofer, R. Steitz, V. Oberst, U. Geckle, M. Bruns and H. Schmidt, The Journal of Physical Chemistry C, 2014, 118, 9395-9399.

187. B. Jerliu, E. Hüger, M. Horisberger, J. Stahn and H. Schmidt, J. Power Sources, 2017, 359, 415-421.

188. B. Jerliu, E. Hüger, L. Dörrer, B. K. Seidlhofer, R. Steitz, M. Horisberger and H. Schmidt, PCCP, 2018, 20, 23480-23491.

189. B.-K. Seidlhofer, B. Jerliu, M. Trapp, E. Hueger, S. Risse, R. Cubitt, H. Schmidt, R. Steitz and M. Ballauff, Acs Nano, 2016, 10, 7458-7466.

190. B.-K. Seidlhofer, B. Jerliu, M. Trapp, E. Hüger, S. Risse, R. Cubitt, H. Schmidt, R. Steitz and M. Ballauff, ACS Nano, 2016, 10, 7458-7466.

191. F. Sun, H. Markoetter, I. Manke, A. Hilger, S. S. Alrwashdeh, N. Kardjilov and J. Banhart, Appl. Surf. Sci., 2017, 399, 359366.

192. R. G. Downing, G. P. Lamaze, J. K. Langland and S. T. Hwang, Journal of research of the National Institute of Standards and Technology, 1993, 98, 109-126.

193. C. Chen, J. F. M. Oudenhoven, D. L. Danilov, E. Vezhlev, L. Gao, N. Li, F. M. Mulder, R.-A. Eichel and P. H. L. Notten, Advanced Energy Materials, 2018, 8, 1870134.

194. D. X. Liu, J. Wang, K. Pan, J. Qiu, M. Canova, L. R. Cao and A. C. Co, Angew. Chem. Int. Ed., 2014, 53, 9498-9502.

195. J. Wang, D. X. Liu, M. Canova, R. G. Downing, L. R. Cao and A. C. Co, J. Radioanal. Nucl. Chem., 2014, 301, 277-284.
D. X. Liu, L. R. Cao and A. C. Co, Chem. Mater., 2016, 28, 556-563.

C. Wang, Y. Gong, J. Dai, L. Zhang, H. Xie, G. Pastel, B. Liu, E. Wachsman, H. Wang and L. Hu, J. Am. Chem. Soc., 2017, 139, 14257-14264.

198. F. Blanc, M. Leskes and C. P. Grey, Acc. Chem. Res., 2013, 46, 1952-1963.

199. J. Z. Hu, N. R. Jaegers, M. Y. Hu and K. T. Mueller, J. Phys.: Condens. Matter, 2018, 30, 463001.

200. C. P. Grey and N. Dupré, Chem. Rev., 2004, 104, 4493-4512. 201. W. J. O'Sullivan and J. E. Schirber, Physical Review, 1968, 170, 667-669.

202. R. E. Gerald, R. J. Klingler, G. Sandi, C. S. Johnson, L. G. Scanlon and J. W. Rathke, J. Power Sources, 2000, 89, 237243.

203. F. Chevallier, M. Letellier, M. Morcrette, J. M. Tarascon, E. Frackowiak, J. N. Rouzaud and F. Beguin, Electrochemical and Solid State Letters, 2003, 6, A225-A228.

204. M. Letellier, F. Chevallier, C. Clinard, E. Frackowiak, J. N. Rouzaud, F. Beguin, M. Morcrette and J. M. Tarascon, J. Chem. Phys., 2003, 118, 6038-6045.

205. B. Key, R. Bhattacharyya, M. Morcrette, V. Seznéc, J.-M. Tarascon and C. P. Grey, J. Am. Chem. Soc., 2009, 131, 9239-9249.

206. K. Ogata, E. Salager, C. J. Kerr, A. E. Fraser, C. Ducati, A. J. Morris, S. Hofmann and C. P. Grey, Nature Communications, 2014, 5.

207. A. I. Freytag, A. D. Pauric, M. Jiang and G. R. Goward, Journal of Physical Chemistry C, 2019, 123, 11362-11368.

R. Bhattacharyya, B. Key, H. Chen, A. S. Best, A. F. Hollenkamp and C. P. Grey, Nature Materials, 2010, 9, 504510.

209. H. J. Chang, N. M. Trease, A. J. Ilott, D. Zeng, L.-S. Du, A. Jerschow and C. P. Grey, Journal of Physical Chemistry C, 2015, 119, 16443-16451.

210. P. M. Bayley, N. M. Trease and C. P. Grey, J. Am. Chem. Soc., 2016, 138, 1955-1961.

211. J. M. Stratford, M. Mayo, P. K. Allan, O. Pecher, O. J. Borkiewicz, K. M. Wiaderek, K. W. Chapman, C. J. Pickard, A. J. Morris and C. P. Grey, J. Am. Chem. Soc., 2017, 139, 7273-7286.

212. M. Nakayama, S. Wada, S. Kuroki and M. Nogami, Energy \& Environmental Science, 2010, 3, 1995-2002.

213. S. Chandrashekar, N. M. Trease, H. J. Chang, L.-S. Du, C. P. Grey and A. Jerschow, Nature Materials, 2012, 11, 311315.

214. A. J. llott, M. Mohammadi, H. J. Chang, C. P. Grey and A. Jerschow, Proceedings of the National Academy of Sciences of the United States of America, 2016, 113, 10779-10784.

215. M. T. Sougrati, J. Fullenwarth, A. Debenedetti, B. Fraisse, J. C. Jumas and L. Monconduit, J. Mater. Chem., 2011, 21, 10069-10076.

216. M. Sathiya, J. B. Leriche, E. Salager, D. Gourier, J. M. Tarascon and H. Vezin, Nature Communications, 2015, 6, 6276.

217. J. Wandt, C. Marino, H. A. Gasteiger, P. Jakes, R.-A. Eichel and J. Granwehr, Energy \& Environmental Science, 2015, 8, 1358-1367.

218. K. Rhodes, N. Dudney, E. Lara-Curzio and C. Daniel, J. Electrochem. Soc., 2010, 157, A1354-A1360.

219. T. Ohzuku, H. Tomura and K. Sawai, J. Electrochem. Soc., 1997, 144, 3496-3500. 
220. C. Villevieille, M. Boinet and L. Monconduit, Electrochem. Commun., 2010, 12, 1336-1339.

221. T. Ohzuku, N. Matoba and K. Sawai, J. Power Sources, 2001, 97-98, 73-77.

222. A. M, PhD thesis, Université Nantes, 2008.

223. S. Kalnaus, K. Rhodes and C. Daniel, J. Power Sources, 2011, 196, 8116-8124.

224. A. Schiele, B. Breitung, A. Mazilkin, S. Schweidler, J. Janek, S. Gumbel, S. Fleischmann, E. Burakowska-Meise, H. Sommer and T. Brezesinski, ACS Omega, 2018, 3, 1670616713.

225. J.-T. Li, J. Światowska, V. Maurice, A. Seyeux, L. Huang, S.G. Sun and P. Marcus, The Journal of Physical Chemistry C, 2011, 115, 7012-7018.

226. E. Hueger, B. Jerliu, L. Doerrer, M. Bruns, G. Borchardt and H. Schmidt, Zeitschrift Fur Physikalische ChemieInternational Journal of Research in Physical Chemistry \& Chemical Physics, 2015, 229, 1375-1385.

227. A. Bordes, E. De Vito, C. Haon, A. Boulineau, A. Montani and P. Marcus, Chem. Mater., 2016, 28, 1566-1573.

228. E. Radvanyi, E. De Vito, W. Porcher, J. Danet, P. Desbois, J.F. Colin and S. J. Si Larbi, Journal of Materials Chemistry A, 2013, 1, 4956-4965.

229. N. Shpigel, M. D. Levi, S. Sigalov, L. Daikhin and D. Aurbach, Acc. Chem. Res., 2018, 51, 69-79.

230. Z. Yang, B. J. Ingram and L. Trahey, J. Electrochem. Soc., 2014, 161, A1127-A1131.

231. Z.-W. Yin, X.-X. Peng, J.-T. Li, C.-H. Shen, Y.-P. Deng, Z.-G. Wu, T. Zhang, Q.-B. Zhang, Y.-X. Mo, K. Wang, L. Huang, H. Zheng and S.-G. Sun, ACS Applied Materials \& Interfaces, 2019, 11, 16214-16222.

232. S. Yagi, M. Fukuda, R. Makiura, T. Ichitsubo and E. Matsubara, Journal of Materials Chemistry A, 2014, 2, 8041-8047.

233. N. Shpigel, M. D. Levi, S. Sigalov, O. Girshevitz, D. Aurbach, L. Daikhin, N. Jäckel and V. Presser, Angew. Chem. Int. Ed., 2015, 54, 12353-12356.

234. Z. Yang, M. C. Dixon, R. A. Erck and L. Trahey, ACS Applied Materials \& Interfaces, 2015, 7, 26585-26594.

235. N. Shpigel, M. D. Levi, S. Sigalov, O. Girshevitz, D. Aurbach, L. Daikhin, P. Pikma, M. Marandi, A. Jänes, E. Lust, N. Jäckel and V. Presser, Nature Materials, 2016, 15, 570.

236. V. Dargel, N. Shpigel, S. Sigalov, P. Nayak, M. D. Levi, L. Daikhin and D. Aurbach, Nature Communications, 2017, 8 1389.

237. P. G. Kitz, M. J. Lacey, P. Novák and E. J. Berg, Anal. Chem., 2019, 91, 2296-2303.

238. M. Yao, P. Wu, S. Cheng, L. Yang, Y. Zhu, M. Wang, H. Luo, B. Wang, D. Ye and M. Liu, PCCP, 2017, 19, 24689-24695.

239. A. Metrot, P. Willmann and A. Herold, Materials Science and Engineering, 1977, 31, 83-86.

240. A. Tranchot, H. Idrissi, P. X. Thivel and L. Roué, J. Power Sources, 2016, 330, 253-260.

241. M. Hahn, H. Buqa, P. W. Ruch, D. Goers, M. E. Spahr, J. Ufheil, P. Novák and R. Kötz, Electrochem. Solid-State Lett., 2008, 11, A151.

242. G. Jeong, S. M. Lee, N. S. Choi, Y.-U. Kim and C. K. Lee, Electrochim. Acta, 2011, 56, 5095-5101.

243. H.-Y. Lin, C.-H. Li, D.-Y. Wang and C.-C. Chen, Nanoscale, 2016, 8, 1280-1287.

244. H. Pan, J. Zhang, Y. Chen, X. Zhuo and Y. Yang, Thin Solid Films, 2010, 519, 778-783.
245.

246.

247.

248.

249

250.

251.

252.

52.

253.
S. Park, T. Kim and S. M. Oh, Electrochem. Solid-State Lett., 2007, 10, A142.

S. S. Hwang, M. Sohn, H.-I. Park, J.-M. Choi, C. G. Cho and H. Kim, Electrochim. Acta, 2016, 211, 356-363.

J. L. Gómez-Cámer, C. Bünzli, M. M. Hantel, T. Poux and P. Novák, Carbon, 2016, 105, 42-51.

D. Y. W. Yu, M. Zhao and H. E. Hoster, ChemElectroChem, 2015, 2, 1090-1095.

S. Wang, X. Yang, P.-K. Lee, A. L. Rogach and D. Y. W. Yu, Chem. Mater., 2019, 31, 2469-2475.

W. Brehm, J. R. Buchheim and P. Adelhelm, Energy Technology, 2019, 7, 1900389.

J. O. Besenhard, J. Yang and M. Winter, J. Power Sources, 1997, 68, 87-90.

M. Zhang, A. L. Gui, W. Sun, J. Becking, O. Riedel, X. He, D. Berghus, V. Siozios, D. Zhou, T. Placke, M. Winter and P. Bieker, J. Electrochem. Soc., 2019, 166, A2142-A2150.

2019, 164, 52-56. 